FR-PHENO-2010-020

PSI-PR-10-10

\title{
Scalar one-loop 4-point integrals
}

\author{
A. Denner ${ }^{1}$ And S. Dittmaier ${ }^{2}$ \\ ${ }^{1}$ Paul Scherrer Institut, Würenlingen und Villigen \\ CH-5232 Villigen PSI, Switzerland \\ 2 Albert-Ludwigs-Universität Freiburg, Physikalisches Institut, \\ D-79104 Freiburg, Germany
}

\begin{abstract}
:
We provide a complete set of results for the scalar 4-point function appearing in oneloop calculations in QCD, QED, the electroweak Standard Model and popular extensions thereof. Complex internal masses, which are needed for calculations involving unstable particles, are supported throughout, whereas complex momenta are not supported. In particular, for the most general, regular case we present two independent results in terms of 72 and 32 dilogarithms. In addition we list explicit results for all soft- and/or collinearsingular cases in dimensional regularization, mass regularization, and in regularizations of mixed type. The exceptional case with a vanishing modified Cayley determinant, which hardly appears in applications, is not considered.
\end{abstract}

May 2010 


\section{Introduction}

Experimental tests of the Standard Model of particle physics or its extensions require precise theoretical predictions. These are to a large extent obtained in perturbation theory. For an adequate investigation of most processes at the LHC or future particle colliders typically at least the next-to-leading order (NLO) in QCD must be known, but for many processes also electroweak one-loop corrections are required. Various interesting processes involve three or more particles in the final state and internal resonances.

In recent years the particle theory community made a big effort to calculate processes for the LHC at NLO. In this context, the traditional approach of evaluating Feynman diagrams has been further developed (see, e.g., Ref. [1, 2, 3, 4] and references therein), and new methods, which abandon the use of individual diagrams, have been proposed and applied [ 5, 6, 7, 8, 9]. Details on these developments and more references to methods and results can, e.g., be found in Ref. [10]. Apart from purely numerical approaches [11, 12, 13, 14, 15], independent of the actual method, finally at the one-loop order all results are expressed as linear combinations of a set of master integrals, typically the standard scalar one-loop integrals 1 In four space-time dimensions [ [17, 18], and in dimensional regularization near four dimensions [ 19], only the scalar 1-point, 2-point, 3-point, and 4-point functions are needed, since higher functions can be reduced to those (see references in Ref. [10] for different variants of these decompositions). For the calculation of electroweak corrections to multiparticle processes these are required for the general case, i.e., with arbitrary internal masses and external momenta. For processes involving unstable particles these functions are also needed for complex parameters. To this end, we support complex internal masses while keeping momentum variables real, i.e. we consider the generalisation required by the so-called "complex-mass scheme" [20], which is frequently employed to describe one-loop corrections to processes with particle resonances.

In Ref. [18] pioneering work in the calculation of scalar one-loop integrals has been carried out by 't Hooft and Veltman. For the 1-point, 2-point, and 3-point functions compact explicit expressions are provided that are valid for complex internal masses and arbitrary physical momenta. For the scalar 4-point function with real masses a result in terms of 24 dilogarithms has been derived in Ref. [18], but for complex masses only a sketch for a result in terms of 108 dilogarithms has been given there. This result has been implemented into a FORTRAN code recently [21]. In Ref. [22] a result for the general scalar 4-point function has been derived involving 16 dilogarithms, but this compact result is only valid for real masses.

Besides the regular scalar functions, in most calculations involving massless or nearly massless particles special soft- or collinear-singular cases appear. This is, in particular, the case for NLO calculations in QCD, where usually dimensional regularization is applied, or in QED and the electroweak Standard Model, where the masses of the light leptons are often taken to be infinitesimally small. For the 2-point function, the singular limits are easy to extract, and for the 3-point function the singular cases have been summarised in Ref. [23]. However, for the 4-point functions a complete set exists only in dimensional

\footnotetext{
${ }^{1}$ Other methods that reduce to a different set of basic integrals also rely in general on numerical evaluation of the basic integrals [16].
} 
regularization so far. The scalar 4-point function with vanishing internal masses and no or one non-zero external mass (in addition to the two non-vanishing Mandelstam variables) is provided in Ref. [24, 25]. All cases with vanishing internal masses are given in Refs. [19, 26, 27]. Finally, using also various other special cases spread in the literature, all singular cases relevant in dimensional regularization have been collected in Ref. [28].

The purpose of this paper is to provide a complete set of formulas for the scalar 4-point function valid for complex masses and in all regular and soft- and/or collinearsingular cases both in dimensional and in mass regularization. In detail, we provide two independent results for the scalar 4-point function with arbitrary complex squared masses (with negative imaginary parts), which are both valid in all kinematical regions relevant for physical processes. One of these results is obtained similar to the method of 't Hooft and Veltman [18] and involves 72 dilogarithms, the other result follows the strategy of Ref. [22], leading to only 32 dilogarithms. For several specific choices of complex masses and kinematical invariants we have numerically compared our results to the ones obtained with the code D0C [ 21], which is based on 108 dilogarithms, and have found agreement. Moreover, we provide complete analytic continuations of the results of Ref. [22] for the cases involving one or more zero masses. Additionally we summarise results for the scalar 4-point function for soft- and collinear-singular cases in dimensional regularization, in mass regularization, and in regularizations of mixed type. As only restriction we require that particles with infinitesimally small masses do not change their mass when emitting a massless particle. This is the case for all applications in QCD, QED, the electroweak Standard Model and popular extensions thereof.

We have derived the singular cases in two different ways. The first method is based on the approach of Ref. [23]. Here, a singular integral is explicitly calculated in a suitable regularization scheme and then translated to all other regularization schemes by adding and subtracting appropriate 3-point functions. As an independent check, many integrals have been directly evaluated in addition, others have been taken from the literature. Specifically, those that do not require dimensional regularization have been derived as limiting cases using the methods and results of Ref. [22], those that require dimensional regularization have been taken from Ref. [28] or from other papers, or derived as special cases therefrom. We have taken care to provide concise results that are valid in all relevant regions of phase space.

However, we do not provide results for exceptional phase-space points, where the denominator in front of the dilogarithms and logarithms vanishes. These cases do only rarely appear in practical calculations, and can often be avoided by introducing a finite width for unstable particles (for examples see Refs. [29, 30]). Either they correspond to Landau singularities, where the scalar 4-point function is singular, or to exceptional non-singular cases that practically do not show up in calculations. If needed, they can be derived as limiting cases of our results. We also do not provide results for complex momentum variables that are required for unstable external particles [31].

The paper is organised as follows: In Section 2 we summarise our conventions and notation. Results for the general non-singular case with complex masses and for the corresponding massless cases are derived in Section 3. Results for all soft- and/or collinearsingular cases are listed in Section 4, and Section 5 contains a short summary. 


\section{Conventions and notation}

The one-loop scalar 4-point integral is defined in $D$ space-time dimensions as

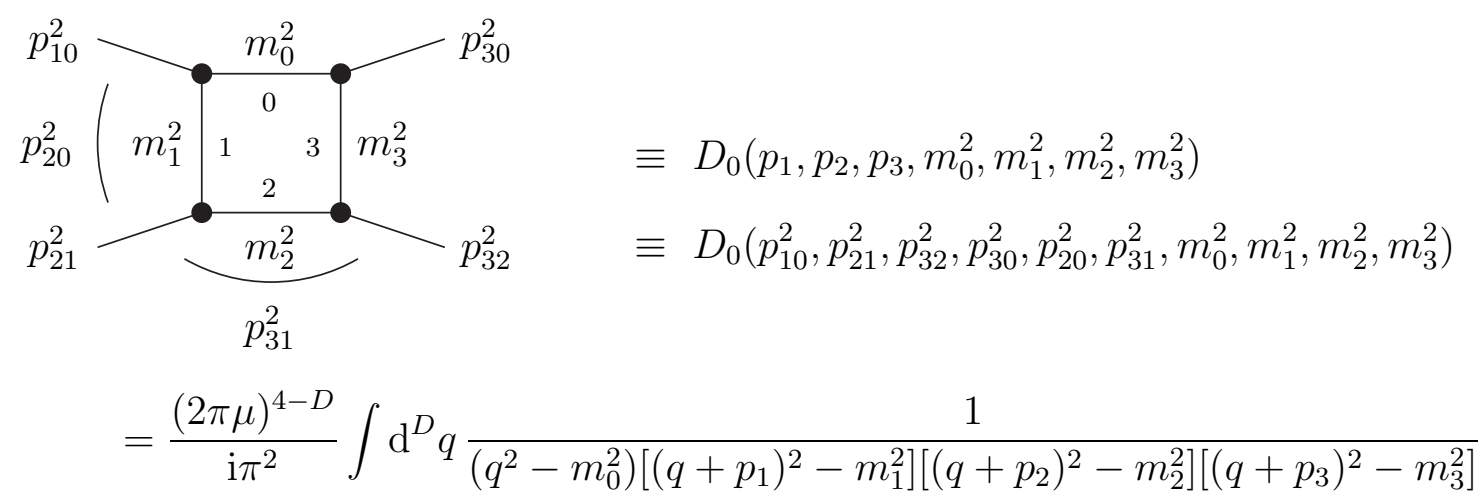

with $p_{0}=0, p_{i j}=p_{i}-p_{j}$, and $\mu$ is the mass scale of dimensional regularization. The internal squared masses $m_{i}^{2}$ are allowed to be complex with phases between 0 and $-\pi / 2$. If not stated otherwise, real squared masses are assumed to have an infinitesimally small negative imaginary part. If $m_{i}$ appears in the following expressions it is defined as the square root of $m_{i}^{2}$ with positive real part. The external momenta are real, but over-lined squared momenta are understood to receive an infinitesimally small positive imaginary part, i.e., $\bar{p}_{i j}^{2}=p_{i j}^{2}+$ i0. Note that for $D \neq 4$ our normalisation deviates from the one used in Refs. [19, 28].

After introducing Feynman parameters and performing the momentum integration (2.1) can be written as

$$
\begin{aligned}
D_{0}= & \Gamma(1+\epsilon)\left(4 \pi \mu^{2}\right)^{\epsilon}(1+\epsilon) \\
& \times \int_{0}^{1} \mathrm{~d} x_{1} \int_{0}^{1-x_{1}} \mathrm{~d} x_{2} \int_{0}^{1-x_{1}-x_{2}} \mathrm{~d} x_{3} \frac{1}{\left[P\left(1-x_{1}-x_{2}-x_{3}, x_{1}, x_{2}, x_{3}\right)\right]^{2+\epsilon}},
\end{aligned}
$$

where $D=4-2 \epsilon$ and the integral extends over the 3 -dimensional unit simplex. The polynomial $P$, which is homogeneous of degree 2 in the four variables $x_{0}, \ldots, x_{3}$, is given by

$$
P\left(x_{0}, x_{1}, x_{2}, x_{3}\right)=\sum_{i=0}^{3} m_{i}^{2} x_{i}^{2}+\sum_{\substack{i, j=0 \\ i<j}}^{3} Y_{i j} x_{i} x_{j}
$$

with the modified Cayley matrix $Y$ defined by

$$
Y_{i j}=Y_{j i}=m_{i}^{2}+m_{j}^{2}-p_{i j}^{2} .
$$

The representation (2.2) shows the symmetry of the 4-point function resulting from permutations of the four internal lines. This symmetry is directly realized in permutations of the indices $\{0,1,2,3\}$ of the squared masses and momenta.

In the results, the roots of quadratic equations

$$
0=m_{i}^{2}+Y_{i j} x+m_{j}^{2} x^{2}=m_{j}^{2}\left(x+\frac{1}{r_{i j, 1}}\right)\left(x+\frac{1}{r_{i j, 2}}\right)=m_{i}^{2}\left(1+r_{i j, 1} x\right)\left(1+r_{i j, 2} x\right)
$$


appear which we denote as $-1 / r_{i j, 1}$ and $-1 / r_{i j, 2}$. It is easy to show that for real momenta and squared masses with phases between 0 and $-\pi / 2$ the variables $r_{i j, k}$ vary only on the first Riemann sheet, i.e., they cannot become real and negative. For real masses this is ensured by the infinitesimal imaginary parts. The sign of the infinitesimal imaginary part of $r_{i j, k}$ resulting from the infinitesimal imaginary parts of the masses can be determined as

$$
\operatorname{sgn}\left(\operatorname{Im} r_{i j, k}\right)=\operatorname{sgn}\left(m_{i}^{2} r_{i j, k}-\frac{m_{j}^{2}}{r_{i j, k}}\right) \quad \text { for } \operatorname{Re}\left(r_{i j, k}\right)<0 \text { and } \operatorname{Im}\left(r_{i j, k}\right)=\text { infinitesimal. }
$$

For negative $r_{i j, k}$, i.e., where the infinitesimal imaginary part matters, an infinitesimal imaginary part of $\bar{p}_{i j}^{2}$ yields the same result for $\operatorname{sgn}\left(\operatorname{Im} r_{i j, k}\right)$.

Instead of using the variables $r_{i j, k}$, we sometimes employ the shorthand

$$
x_{i j}=\frac{\sqrt{1-4 m_{i} m_{j} /\left[\bar{p}_{i j}^{2}-\left(m_{i}-m_{j}\right)^{2}\right]}-1}{\sqrt{1-4 m_{i} m_{j} /\left[\bar{p}_{i j}^{2}-\left(m_{i}-m_{j}\right)^{2}\right]}+1},
$$

which is - ignoring the infinitesimal imaginary part for a moment - related to the roots of (2.5) via $r_{i j, 1}=\left(m_{j} / m_{i}\right) x_{i j}$ and $r_{i j, 2}=\left(m_{j} / m_{i}\right) x_{i j}^{-1}$.

The 4-point function is evaluated in terms of logarithms and dilogarithms. As usual we choose the principal value of the logarithm such that the cut lies along the negative real axis. Hence one finds for $a, b$, and $a b$ not on the negative real axis

$$
\ln (a b)-\ln (a)-\ln (b)=\eta(a, b)
$$

with

$$
\eta(a, b)=2 \pi \mathrm{i}\{\theta(-\operatorname{Im} a) \theta(-\operatorname{Im} b) \theta(\operatorname{Im}(a b))-\theta(\operatorname{Im} a) \theta(\operatorname{Im} b) \theta(-\operatorname{Im}(a b))\} .
$$

The principal value of the dilogarithms for $(1-z)$ not real and negative is given by

$$
\operatorname{Li}_{2}(z)=-\int_{0}^{1} \frac{\mathrm{d} t}{t} \ln (1-z t) .
$$

More details and various useful relations for the dilogarithm can be found in Ref. [ [32].

Most of our results involve only functions of the type

$$
\ln \left(\prod_{i=1}^{n} x_{i}\right), \quad \operatorname{Li}_{2}\left(1-\prod_{i=1}^{n} x_{i}\right)
$$

where the variables $x_{i}$ vary only on the first Riemann sheet, i.e., $-\pi<\arg \left(x_{i}\right)<\pi$. Once these results have been derived in the Euclidean region and for real masses they can be easily analytically continued by the substitutions [33]

$$
\ln \left(\prod_{i=1}^{n} x_{i}\right) \rightarrow \sum_{i=1}^{n} \ln \left(x_{i}\right)
$$




$$
\begin{aligned}
\operatorname{Li}_{2}\left(1-\prod_{i=1}^{n} x_{i}\right) \rightarrow & \mathcal{L} i_{2}\left(x_{1}, \ldots, x_{n}\right) \\
\equiv & \operatorname{Li}_{2}\left(1-\prod_{i=1}^{n} x_{i}\right)+\left[\ln \left(\prod_{i=1}^{n} x_{i}\right)-\sum_{i=1}^{n} \ln \left(x_{i}\right)\right]\left[\ln \left(1-\prod_{i=1}^{n} x_{i}\right)\right. \\
& \left.-\theta\left(\left|\prod_{i=1}^{n} x_{i}\right|-1\right)\left(\ln \left(-\prod_{i=1}^{n} x_{i}\right)-\frac{1}{2} \ln \left(\prod_{i=1}^{n} x_{i}\right)-\frac{1}{2} \sum_{i=1}^{n} \ln \left(x_{i}\right)\right)\right]
\end{aligned}
$$

where $\theta$ is the Heaviside step function. In most of our results we need only the analytically continued dilogarithm for two variables $x_{1}, x_{2}$. This simplifies to

$$
\mathcal{L} i_{2}\left(x_{1}, x_{2}\right)=\operatorname{Li}_{2}\left(1-x_{1} x_{2}\right)+\eta\left(x_{1}, x_{2}\right) \ln \left(1-x_{1} x_{2}\right),
$$

because the term proportional to the $\theta$-function in (2.13) does not contribute for $n=2$ if the variables $x_{i}$ stay on the first Riemann sheet.

\section{Regular 4-point integrals}

\subsection{4-point integral for general complex masses - method similar to 't Hooft's and Veltman's}

In this section we evaluate the general scalar 4-point integral $D_{0}$ with complex internal masses $m_{i}$ widely following the method of Ref. [18]. As stated above, we implicitly assume that the mass squares $m_{i}^{2}$ possess either a non-zero negative imaginary part or an infinitesimally small imaginary part that is negative. Moreover, in this section we use the fact the the real part of $m_{i}^{2}$ is non-negative (a restriction that is relaxed in the next section). The integral is both UV and IR finite, so that we set $D=4$ from the beginning, and (2.2) simplifies to

$$
D_{0}=\int_{0}^{1} \mathrm{~d} x_{1} \int_{0}^{1-x_{1}} \mathrm{~d} x_{2} \int_{0}^{1-x_{1}-x_{2}} \mathrm{~d} x_{3} \frac{1}{\left[P\left(1-x_{1}-x_{2}-x_{3}, x_{1}, x_{2}, x_{3}\right)\right]^{2}}
$$

with the polynomial $P$ as defined in (2.3). For later convenience we define the polynomials

$$
\begin{aligned}
P_{k}\left(x_{0}, x_{1}, x_{2}, x_{3}\right) & \equiv \frac{\partial}{\partial x_{k}} P\left(x_{0}, x_{1}, x_{2}, x_{3}\right)=2 m_{k}^{2} x_{k}+\sum_{\substack{i=0 \\
i \neq k}}^{3} Y_{i k} x_{i}=\sum_{i=0}^{3} Y_{i k} x_{i}, \\
k=0, \ldots, 3, & \\
P_{a}\left(x_{0}, x_{1}, x_{2}, x_{3} ; \alpha\right) & \equiv\left[-(1+\alpha) P_{0}+\alpha P_{2}+P_{3}\right]\left(x_{0}, x_{1}, x_{2}, x_{3}\right), \\
P_{b}\left(x_{0}, x_{1}, x_{2}, x_{3} ; \beta\right) & \equiv\left[-(1+\beta) P_{0}+P_{1}+\beta P_{2}\right]\left(x_{0}, x_{1}, x_{2}, x_{3}\right),
\end{aligned}
$$

which are linear and homogeneous in the $x_{i}$, and the real-valued function

$$
\begin{aligned}
P_{a b}(\alpha, \beta)= & 2(1+\alpha)(1+\beta) m_{0}^{2}-(1+\alpha) Y_{01}-(\alpha+\beta+2 \alpha \beta) Y_{02}-(1+\beta) Y_{03} \\
& +\alpha Y_{12}+Y_{13}+2 \alpha \beta m_{2}^{2}+\beta Y_{23} \\
= & (1+\alpha) p_{10}^{2}+(\alpha+\beta+2 \alpha \beta) p_{20}^{2}+(1+\beta) p_{30}^{2}-\alpha p_{21}^{2}-p_{31}^{2}-\beta p_{32}^{2},
\end{aligned}
$$

with $\alpha$ and $\beta$ being real parameters. 

to

Before performing the first integration, we rescale the integration parameters according

$$
x_{2}=\left(1-x_{1}\right) \xi_{2}, \quad x_{3}=\left(1-x_{1}\right) \xi_{3},
$$

so that the non-trivial part of the integration boundary simplifies to the 2-dimensional unit simplex,

$$
D_{0}=\int_{0}^{1} \mathrm{~d} x_{1} \int_{0}^{1} \mathrm{~d} \xi_{2} \int_{0}^{1-\xi_{2}} \mathrm{~d} \xi_{3} \frac{\left(1-x_{1}\right)^{2}}{\left[P\left(\left(1-x_{1}\right)\left(1-\xi_{2}-\xi_{3}\right), x_{1},\left(1-x_{1}\right) \xi_{2},\left(1-x_{1}\right) \xi_{3}\right)\right]^{2}}
$$

Now we perform the "Euler shift"

$$
\xi_{2}=y_{2}+\alpha \xi_{3}
$$

in order to substitute variable $\xi_{2}$ by $y_{2}$. The parameter $\alpha$ is chosen to obey the condition

$$
0=p_{30}^{2}+\alpha\left(p_{30}^{2}-p_{32}^{2}+p_{20}^{2}\right)+\alpha^{2} p_{20}^{2}
$$

which renders to coefficient of $\xi_{3}^{2}$ in $P$ vanishing. At this point it is important to realize that the parameter $\alpha$ is a real quantity for all possible momentum configurations occurring in any scattering or decay (sub)process. For the next intermediate steps we assume $\alpha>0$ and analytically continue the final result to arbitrary real $\alpha$ later. For $\alpha>0$ the integration over $\xi_{2}$ and $\xi_{3}$ can be written as

$$
\begin{aligned}
\int_{0}^{1} \mathrm{~d} \xi_{2} \int_{0}^{1-\xi_{2}} \mathrm{~d} \xi_{3} & =\int_{0}^{1} \mathrm{~d} \xi_{3} \int_{-\alpha \xi_{3}}^{1-(1+\alpha) \xi_{3}} \mathrm{~d} y_{2} \\
& =\int_{0}^{-\alpha} \mathrm{d} y_{2} \int_{0}^{-y_{2} / \alpha} \mathrm{d} \xi_{3}+\int_{-\alpha}^{1} \mathrm{~d} y_{2} \int_{0}^{\left(1-y_{2}\right) /(1+\alpha)} \mathrm{d} \xi_{3} .
\end{aligned}
$$

Exploiting the linearity of $P$ in $\xi_{3}$, the $\xi_{3}$ dependence can be isolated,

$$
P\left(\left(1-x_{1}\right)\left(1-y_{2}-(1+\alpha) \xi_{3}\right), x_{1},\left(1-x_{1}\right)\left(y_{2}+\alpha \xi_{3}\right),\left(1-x_{1}\right) \xi_{3}\right)=\left(1-x_{1}\right) \xi_{3} A+B
$$

with

$$
\begin{aligned}
& A \equiv A\left(x_{1}, y_{2}\right)=P_{a}\left(\left(1-x_{1}\right)\left(1-y_{2}\right), x_{1},\left(1-x_{1}\right) y_{2}, 0 ; \alpha\right) \\
& B \equiv B\left(x_{1}, y_{2}\right)=P\left(\left(1-x_{1}\right)\left(1-y_{2}\right), x_{1},\left(1-x_{1}\right) y_{2}, 0\right)
\end{aligned}
$$

The $\xi_{3}$-integration is carried out as follows,

$$
\begin{aligned}
D_{0}= & \int_{0}^{1} \mathrm{~d} x_{1}\left\{\int_{0}^{-\alpha} \mathrm{d} y_{2} \int_{0}^{-y_{2} / \alpha} \mathrm{d} \xi_{3}+\int_{-\alpha}^{1} \mathrm{~d} y_{2} \int_{0}^{\left(1-y_{2}\right) /(1+\alpha)} \mathrm{d} \xi_{3}\right\} \frac{\left(1-x_{1}\right)^{2}}{\left[\left(1-x_{1}\right) \xi_{3} A+B\right]^{2}} \\
= & -\int_{0}^{1} \mathrm{~d} x_{1} \int_{0}^{-\alpha} \mathrm{d} y_{2} \frac{\alpha\left(1-x_{1}\right)}{A\left[-\left(1-x_{1}\right) y_{2} A+\alpha B\right]} \\
& -\int_{0}^{1} \mathrm{~d} x_{1} \int_{-\alpha}^{1} \mathrm{~d} y_{2} \frac{(1+\alpha)\left(1-x_{1}\right)}{A\left[\left(1-x_{1}\right)\left(1-y_{2}\right) A+(1+\alpha) B\right]}+\int_{0}^{1} \mathrm{~d} x_{1} \int_{0}^{1} \mathrm{~d} y_{2} \frac{1-x_{1}}{A B} .
\end{aligned}
$$


In the last integral we combined the two contributions resulting from the boundary $\xi_{3}=0$ to a single integral using $\int_{0}^{-\alpha} \mathrm{d} y_{2}+\int_{-\alpha}^{1} \mathrm{~d} y_{2}=\int_{0}^{1} \mathrm{~d} y_{2}$. We perform the following three different substitutions in the three integrals of (3.11), respectively,

$$
y_{2}=-\alpha+\frac{\alpha \eta_{2}}{1-x_{1}}, \quad y_{2}=1-\frac{(1+\alpha) \eta_{2}}{1-x_{1}}, \quad y_{2}=\frac{\eta_{2}}{1-x_{1}}
$$

and introduce the shorthands $A_{i}$ and $B_{i}$ for the functions of $x_{1}$ and $\eta_{2}$ that result from $A\left(x_{1}, y_{2}\right)$ and $B\left(x_{1}, y_{2}\right)$ upon making the three substitutions (3.12) that are successively labelled by $i=1,2,3$. The functions $A_{i}$ and $B_{i}$ are polynomials in $x_{1}$ and $\eta_{2}$, in particular, the $A_{i}$ are of linear and the $B_{i}$ of quadratic degree. This is explicit after expressing them in terms of $P$,

$$
\begin{aligned}
& A_{1} \equiv A_{1}\left(x_{1}, \eta_{2}\right)=P_{a}\left((1+\alpha)\left(1-x_{1}\right)-\alpha \eta_{2}, x_{1},-\alpha\left(1-x_{1}-\eta_{2}\right), 0 ; \alpha\right), \\
& A_{2} \equiv A_{2}\left(x_{1}, \eta_{2}\right)=P_{a}\left((1+\alpha) \eta_{2}, x_{1}, 1-x_{1}-(1+\alpha) \eta_{2}, 0 ; \alpha\right), \\
& A_{3} \equiv A_{3}\left(x_{1}, \eta_{2}\right)=P_{a}\left(1-x_{1}-\eta_{2}, x_{1}, \eta_{2}, 0 ; \alpha\right), \\
& B_{1} \equiv B_{1}\left(x_{1}, \eta_{2}\right)=P\left((1+\alpha)\left(1-x_{1}\right)-\alpha \eta_{2}, x_{1},-\alpha\left(1-x_{1}-\eta_{2}\right), 0\right), \\
& B_{2} \equiv B_{2}\left(x_{1}, \eta_{2}\right)=P\left((1+\alpha) \eta_{2}, x_{1}, 1-x_{1}-(1+\alpha) \eta_{2}, 0\right), \\
& B_{3} \equiv B_{3}\left(x_{1}, \eta_{2}\right)=P\left(1-x_{1}-\eta_{2}, x_{1}, \eta_{2}, 0\right) .
\end{aligned}
$$

The $D_{0}$ integral becomes

$$
D_{0}=\int_{0}^{1} \mathrm{~d} x_{1} \int_{0}^{1-x_{1}} \mathrm{~d} \eta_{2}\left\{\frac{\alpha}{A_{1}\left[\left(1-x_{1}-\eta_{2}\right) A_{1}+B_{1}\right]}-\frac{1+\alpha}{A_{2}\left[\eta_{2} A_{2}+B_{2}\right]}+\frac{1}{A_{3} B_{3}}\right\} .
$$

In this representation it is obvious that the contributions involving $A_{1}, B_{1}$ or $A_{2}, B_{2}$ are zero in the special cases $\alpha=0$ or $\alpha=-1$, respectively. In order to eliminate the $x_{1}^{2}$ terms in the factors containing $B_{i}$ in (3.14), we again introduce Euler shifts

$$
\eta_{2}=z+\beta_{i} x_{1}
$$

with three different variables $\beta_{i}$ which correspond to the parts containing $A_{i}$ and $B_{i}$. The following conditions render the above-mentioned coefficients of $x_{1}^{2}$ zero, but at the same time lead to real-valued values of $\beta_{i}$ (for physical decay and scattering processes),

$$
\begin{aligned}
& 0=p_{31}^{2}+\beta_{1}\left(p_{31}^{2}-p_{10}^{2}+p_{30}^{2}\right)+\beta_{1}^{2} p_{30}^{2}, \\
& 0=p_{21}^{2}+\beta_{2}\left(p_{21}^{2}-p_{31}^{2}+p_{32}^{2}\right)+\beta_{2}^{2} p_{32}^{2}, \\
& 0=p_{10}^{2}+\beta_{3}\left(p_{10}^{2}-p_{21}^{2}+p_{20}^{2}\right)+\beta_{3}^{2} p_{20}^{2} .
\end{aligned}
$$

For intermediate steps we again impose the condition $\beta_{i}>0$ and perform analytical continuations to arbitrary real $\beta_{i}$ at the end. Having eliminated the $x_{1}^{2}$ terms in the $B_{i}$ parts of (3.14), each of the three terms in curly brackets can be decomposed via partial fractioning in $x_{1}$, so that only terms of the form $1 /\left(a x_{1}+b\right)$ with $x_{1}$-independent terms $a$ and $b$ result. To make this step explicit, we isolate the $x_{1}$ terms in $A_{i}$ and $B_{i}$,

$$
\begin{aligned}
& A_{i}\left(x_{1}, z+\beta_{i} x_{1}\right)=A_{i}^{(0)}(z)+x_{1} A_{i}^{(1)}(z), \\
& B_{i}\left(x_{1}, z+\beta_{i} x_{1}\right)=B_{i}^{(0)}(z)+x_{1} B_{i}^{(1)}(z)+x_{1}^{2} B_{i}^{(2)}(z) .
\end{aligned}
$$


The arguments of $A_{i}^{(k)}$ and $B_{i}^{(k)}$ are suppressed in the following. The coefficients $B_{i}^{(2)}$ are fixed by the above elimination of $x_{1}^{2}$ terms,

$$
B_{1}^{(2)}=\left(1+\beta_{1}\right) A_{1}^{(1)}, \quad B_{2}^{(2)}=-\beta_{2} A_{2}^{(1)}, \quad B_{3}^{(2)}=0,
$$

and drop out in the subsequent manipulations. The other coefficients read

$$
\begin{aligned}
& A_{1}^{(0)}=P_{a}(1+\alpha(1-z), 0,-\alpha(1-z), 0 ; \alpha), \quad A_{1}^{(1)}=P_{a b}\left(\alpha, \alpha\left(1+\beta_{1}\right)\right), \\
& B_{1}^{(0)}=P(1+\alpha(1-z), 0,-\alpha(1-z), 0), \\
& B_{1}^{(1)}=P_{b}\left(1+\alpha(1-z), 0,-\alpha(1-z), 0 ; \alpha\left(1+\beta_{1}\right)\right), \\
& A_{2}^{(0)}=P_{a}((1+\alpha) z, 0,1-(1+\alpha) z, 0 ; \alpha), \quad A_{2}^{(1)}=P_{a b}\left(\alpha,-1-(1+\alpha) \beta_{2}\right), \\
& B_{2}^{(0)}=P((1+\alpha) z, 0,1-(1+\alpha) z, 0), \\
& B_{2}^{(1)}=P_{b}\left((1+\alpha) z, 0,1-(1+\alpha) z, 0 ;-1-(1+\alpha) \beta_{2}\right), \\
& A_{3}^{(0)}=P_{a}(1-z, 0, z, 0 ; \alpha), \quad A_{3}^{(1)}=P_{a b}\left(\alpha, \beta_{3}\right), \\
& B_{3}^{(0)}=P(1-z, 0, z, 0), \quad B_{3}^{(1)}=P_{b}\left(1-z, 0, z, 0 ; \beta_{3}\right) .
\end{aligned}
$$

The partial fractioning of the integrand in (3.14) with respect to its $x_{1}$ dependence yields

$$
\begin{aligned}
& \frac{1}{C_{1}(z)}\left[\left(x_{1}+\frac{A_{1}^{(0)}}{A_{1}^{(1)}}\right)^{-1}-\left(x_{1}+\frac{(1-z) A_{1}^{(0)}+B_{1}^{(0)}}{-\left(1+\beta_{1}\right) A_{1}^{(0)}+(1-z) A_{1}^{(1)}+B_{1}^{(1)}}\right)^{-1}\right] \\
& +\frac{1}{C_{2}(z)}\left[\left(x_{1}+\frac{A_{2}^{(0)}}{A_{2}^{(1)}}\right)^{-1}-\left(x_{1}+\frac{B_{2}^{(0)}+z A_{2}^{(0)}}{\beta_{2} A_{2}^{(0)}+B_{2}^{(1)}+z A_{2}^{(1)}}\right)^{-1}\right] \\
& +\frac{1}{C_{3}(z)}\left[\left(x_{1}+\frac{A_{3}^{(0)}}{A_{3}^{(1)}}\right)^{-1}-\left(x_{1}+\frac{B_{3}^{(0)}}{B_{3}^{(1)}}\right)^{-1}\right]
\end{aligned}
$$

with

$$
\begin{aligned}
& C_{1}(z)=\frac{1}{\alpha}\left[A_{1}^{(1)} B_{1}^{(0)}-A_{1}^{(0)} B_{1}^{(1)}+\left(A_{1}^{(0)}\right)^{2}\left(1+\beta_{1}\right)\right], \\
& C_{2}(z)=\frac{1}{1+\alpha}\left[-A_{2}^{(1)} B_{2}^{(0)}+A_{2}^{(0)} B_{2}^{(1)}+\left(A_{2}^{(0)}\right)^{2} \beta_{2}\right], \\
& C_{3}(z)=A_{3}^{(1)} B_{3}^{(0)}-A_{3}^{(0)} B_{3}^{(1)},
\end{aligned}
$$

which are quadratic polynomials in $z$. In order to carry out the $x_{1}$-integration we manipulate to double integral analogously to (3.8),

$$
\begin{aligned}
\int_{0}^{1} \mathrm{~d} x_{1} \int_{0}^{1-x_{1}} \mathrm{~d} \eta_{2} & =\int_{0}^{1} \mathrm{~d} x_{1} \int_{-\beta_{i} x_{1}}^{1-\left(1+\beta_{i}\right) x_{1}} \mathrm{~d} z \\
& =\int_{0}^{-\beta_{i}} \mathrm{~d} z \int_{0}^{-z / \beta_{i}} \mathrm{~d} x_{1}+\int_{-\beta_{i}}^{1} \mathrm{~d} z \int_{0}^{(1-z) /\left(1+\beta_{i}\right)} \mathrm{d} x_{1} .
\end{aligned}
$$


The result of the integration is

$$
\begin{aligned}
D_{0}= & \int_{0}^{-\beta_{1}} \mathrm{~d} z \frac{1}{C_{1}(z)}\left\{\ln A_{1}\left(-\frac{z}{\beta_{1}}, 0\right)-\ln \left[\left(1+\frac{z}{\beta_{1}}\right) A_{1}\left(-\frac{z}{\beta_{1}}, 0\right)+B_{1}\left(-\frac{z}{\beta_{1}}, 0\right)\right]\right\} \\
& +\int_{0}^{-\beta_{2}} \mathrm{~d} z \frac{1}{C_{2}(z)}\left\{\ln A_{2}\left(-\frac{z}{\beta_{2}}, 0\right)-\ln B_{2}\left(-\frac{z}{\beta_{2}}, 0\right)\right\} \\
& +\int_{0}^{-\beta_{3}} \mathrm{~d} z \frac{1}{C_{3}(z)}\left\{\ln A_{3}\left(-\frac{z}{\beta_{3}}, 0\right)-\ln B_{3}\left(-\frac{z}{\beta_{3}}, 0\right)\right\} \\
& +\int_{-\beta_{1}}^{1} \mathrm{~d} z \frac{1}{C_{1}(z)}\left\{\ln A_{1}\left(\frac{1-z}{1+\beta_{1}}, \frac{z+\beta_{1}}{1+\beta_{1}}\right)-\ln B_{1}\left(\frac{1-z}{1+\beta_{1}}, \frac{z+\beta_{1}}{1+\beta_{1}}\right)\right\} \\
& +\int_{-\beta_{2}}^{1} \mathrm{~d} z \frac{1}{C_{2}(z)}\left\{\ln A_{2}\left(\frac{1-z}{1+\beta_{2}}, \frac{z+\beta_{2}}{1+\beta_{2}}\right)\right. \\
& \left.-\ln \left[\frac{z+\beta_{2}}{1+\beta_{2}} A_{2}\left(\frac{1-z}{1+\beta_{2}}, \frac{z+\beta_{2}}{1+\beta_{2}}\right)+B_{2}\left(\frac{1-z}{1+\beta_{2}}, \frac{z+\beta_{2}}{1+\beta_{2}}\right)\right]\right\} \\
& -\int_{-\beta_{3}}^{1} \mathrm{~d} z \frac{1}{C_{3}(z)}\left\{\ln A_{3}\left(\frac{1-z}{1+\beta_{3}}, \frac{z+\beta_{3}}{1+\beta_{3}}\right)-\ln B_{3}\left(\frac{1-z}{1+\beta_{3}}, \frac{z+\beta_{3}}{1+\beta_{3}}\right)\right\} \\
& -\int_{0}^{1} \mathrm{~d} z \frac{1}{C_{1}(z)}\left\{\ln A_{1}(0, z)-\ln \left[(1-z) A_{1}(0, z)+B_{1}(0, z)\right]\right\} \\
& -\int_{0}^{1} \mathrm{~d} z \frac{1}{C_{2}(z)}\left\{\ln A_{2}(0, z)-\ln \left[z A_{2}(0, z)+B_{2}(0, z)\right]\right\} \\
& -\int_{0}^{1} \mathrm{~d} z \frac{1}{C_{3}(z)}\left\{\ln A_{3}(0, z)-\ln B_{3}(0, z)\right\} .
\end{aligned}
$$

Organising the logarithms in this way, of course, requires some care concerning their cut structures as functions of $z$. For instance, we have evaluated the integrals containing only $A_{i}$ functions as follows,

$$
\begin{aligned}
\int_{r_{0}}^{r_{1}} \mathrm{~d} x_{1}\left(x_{1}+\frac{A_{i}^{(0)}}{A_{i}^{(1)}}\right)^{-1} & =\ln \left(r_{1}+\frac{A_{i}^{(0)}}{A_{i}^{(1)}}\right)-\ln \left(r_{0}+\frac{A_{i}^{(0)}}{A_{i}^{(1)}}\right) \\
& =\ln \left(A_{i}^{(1)} r_{1}+A_{i}^{(0)}\right)-\ln \left(A_{i}^{(1)} r_{0}+A_{i}^{(0)}\right) \\
& =\ln A_{i}\left(r_{1}, z+\beta_{i} r_{1}\right)-\ln A_{i}\left(r_{0}, z+\beta_{i} r_{0}\right),
\end{aligned}
$$

justified by the fact that all $r_{i}$ and $A_{i}^{(1)}$ are real valued, so that the arguments of the two logarithms in the differences have the same imaginary part. Similar manipulations are possible for the integrals containing $B_{i}$ functions after observing that the imaginary parts of $A_{i}^{(0)}$ and $B_{i}^{(1)}$ are $z$-independent and that the signs of the imaginary parts in the resulting logarithms do not change with varying $z$ (i.e. no branch cuts are crossed). Moreover, it can be easily checked that in (3.23) the residues of the zeros of the functions $C_{i}(z)$ add up to zero. This fact would be useful if we proceeded with the final integration as suggested in Ref. [18]; however, we go a somewhat different way and exploit this fact at the very end in the analytical continuation in $\alpha$ and $\beta_{i}$.

For later convenience, we factorize the quadratic polynomials $C_{i}(z)$ as follows,

$$
C_{i}(z)=a_{i} z^{2}+b_{i} z+c_{i}=a_{i}\left(z-z_{i, 1}\right)\left(z-z_{i, 2}\right)
$$


i.e. $z_{i, 1}$ and $z_{i, 2}$ are the solutions of the quadratic equations $C_{i}(z)=0$. The constants $a_{i}$, $b_{i}$, and $c_{i}$ are explicitly given by

$$
\begin{aligned}
& a_{1}=\alpha\left[p_{20}^{2} P_{a b}\left(\alpha, \alpha\left(1+\beta_{1}\right)\right)-P_{a}(-1,0,1,0 ; \alpha) P_{b}\left(-1,0,1,0 ; \alpha\left(1+\beta_{1}\right)\right)\right. \\
& \left.+\left(1+\beta_{1}\right) P_{a}(-1,0,1,0 ; \alpha)^{2}\right], \\
& b_{1}=\left(Y_{20}-2 m_{0}^{2}-2 \alpha p_{20}^{2}\right) P_{a b}\left(\alpha, \alpha\left(1+\beta_{1}\right)\right)-P_{a}(1+\alpha, 0,-\alpha, 0 ; \alpha) \\
& \times P_{b}\left(-1,0,1,0 ; \alpha\left(1+\beta_{1}\right)\right)-P_{a}(-1,0,1,0 ; \alpha) P_{b}\left(1+\alpha, 0,-\alpha, 0 ; \alpha\left(1+\beta_{1}\right)\right) \\
& +2\left(1+\beta_{1}\right) P_{a}(-1,0,1,0 ; \alpha) P_{a}(1+\alpha, 0,-\alpha, 0 ; \alpha), \\
& c_{1}=\frac{1}{\alpha}\left[\left((1+\alpha) Y_{30}-\alpha Y_{23}-m_{3}^{2}\right) P_{a b}\left(\alpha, \alpha\left(1+\beta_{1}\right)\right)-P_{a}(1+\alpha, 0,-\alpha, 0 ; \alpha)\right. \\
& \left.\times P_{b}\left(1+\alpha, 0,-\alpha, 0 ; \alpha\left(1+\beta_{1}\right)\right)+\left(1+\beta_{1}\right) P_{a}(1+\alpha, 0,-\alpha, 0 ; \alpha)^{2}\right], \\
& a_{2}=(1+\alpha)\left[-p_{20}^{2} P_{a b}\left(\alpha,-1-(1+\alpha) \beta_{2}\right)+P_{a}(1,0,-1,0 ; \alpha)\right. \\
& \left.\times P_{b}\left(1,0,-1,0 ;-1-(1+\alpha) \beta_{2}\right)+P_{a}(1,0,-1,0 ; \alpha)^{2} \beta_{2}\right], \\
& b_{2}=-\left(Y_{20}-2 m_{2}^{2}\right) P_{a b}\left(\alpha,-1-(1+\alpha) \beta_{2}\right)+P_{a}(1,0,-1,0 ; \alpha) \\
& \times P_{b}\left(0,0,1,0 ;-1-(1+\alpha) \beta_{2}\right)+P_{a}(0,0,1,0 ; \alpha) P_{b}\left(1,0,-1,0 ;-1-(1+\alpha) \beta_{2}\right) \\
& +2 \beta_{2} P_{a}(1,0,-1,0 ; \alpha) P_{a}(0,0,1,0 ; \alpha), \\
& c_{2}=\frac{1}{1+\alpha}\left[-m_{2}^{2} P_{a b}\left(\alpha,-1-(1+\alpha) \beta_{2}\right)+P_{a}(0,0,1,0 ; \alpha) P_{b}\left(0,0,1,0 ;-1-(1+\alpha) \beta_{2}\right)\right. \\
& \left.+\beta_{2} P_{a}(0,0,1,0, \alpha)^{2}\right] \text {, } \\
& a_{3}=p_{20}^{2} P_{a b}\left(\alpha, \beta_{3}\right)-P_{a}(-1,0,1,0 ; \alpha) P_{b}\left(-1,0,1,0 ; \beta_{3}\right), \\
& b_{3}=\left(Y_{20}-2 m_{0}^{2}\right) P_{a b}\left(\alpha, \beta_{3}\right)-P_{a}(1,0,0,0 ; \alpha) P_{b}\left(-1,0,1,0 ; \beta_{3}\right) \\
& \text { - } P_{a}(-1,0,1,0 ; \alpha) P_{b}\left(1,0,0,0 ; \beta_{3}\right) \text {, } \\
& c_{3}=m_{0}^{2} P_{a b}\left(\alpha, \beta_{3}\right)-P_{a}(1,0,0,0 ; \alpha) P_{b}\left(1,0,0,0 ; \beta_{3}\right),
\end{aligned}
$$

and obey the non-trivial relations

$$
\operatorname{det} Y=b_{i}^{2}-4 a_{i} c_{i}, \quad i=1,2,3,
$$

so that we can define

$$
z_{i, 1}=\frac{-b_{i}+\sqrt{\operatorname{det} Y}}{2 a_{i}}, \quad z_{i, 2}=\frac{-b_{i}-\sqrt{\operatorname{det} Y}}{2 a_{i}} .
$$

In order to simplify the integrand of (3.23), we perform the substitutions

$$
\zeta=-\frac{z}{\beta_{i}}, \quad \zeta=\frac{1-z}{1+\beta_{i}}, \quad \zeta=z
$$

in the three different blocks of integrals $\int_{0}^{-\beta_{i}} \mathrm{~d} z, \int_{-\beta_{i}}^{1} \mathrm{~d} z, \int_{0}^{1} \mathrm{~d} z$, respectively, leading to a common range of integration over $\zeta$, viz. $\int_{0}^{1} \mathrm{~d} \zeta$. This transforms the $z_{i, j}$ into

$$
\zeta_{i, j}^{\prime}=-\frac{z_{i, j}}{\beta_{i}}, \quad \zeta_{i, j}^{\prime \prime}=\frac{1-z_{i, j}}{1+\beta_{i}}, \quad \zeta_{i, j}=z_{i, j}
$$


and integral (3.23) becomes

$$
\begin{aligned}
D_{0}= & \frac{1}{\sqrt{\operatorname{det} Y}} \int_{0}^{1} \mathrm{~d} \zeta \\
\times\{[ & \left.\left(\zeta-\zeta_{1,1}^{\prime}\right)^{-1}-\left(\zeta-\zeta_{1,2}^{\prime}\right)^{-1}\right]\left\{\ln A_{1}(\zeta, 0)-\ln \left[(1-\zeta) A_{1}(\zeta, 0)+B_{1}(\zeta, 0)\right]\right\} \\
& +\left[\left(\zeta-\zeta_{2,1}^{\prime}\right)^{-1}-\left(\zeta-\zeta_{2,2}^{\prime}\right)^{-1}\right]\left\{\ln A_{2}(\zeta, 0)-\ln B_{2}(\zeta, 0)\right\} \\
+ & {\left[\left(\zeta-\zeta_{3,1}^{\prime}\right)^{-1}-\left(\zeta-\zeta_{3,2}^{\prime}\right)^{-1}\right]\left\{\ln A_{3}(\zeta, 0)-\ln B_{3}(\zeta, 0)\right\} } \\
- & {\left[\left(\zeta-\zeta_{1,1}^{\prime \prime}\right)^{-1}-\left(\zeta-\zeta_{1,2}^{\prime \prime}\right)^{-1}\right]\left\{\ln A_{1}(\zeta, 1-\zeta)-\ln B_{1}(\zeta, 1-\zeta)\right\} } \\
- & {\left[\left(\zeta-\zeta_{2,1}^{\prime \prime}\right)^{-1}-\left(\zeta-\zeta_{2,2}^{\prime \prime}\right)^{-1}\right] } \\
& \times\left\{\ln A_{2}(\zeta, 1-\zeta)-\ln \left[(1-\zeta) A_{2}(\zeta, 1-\zeta)+B_{2}(\zeta, 1-\zeta)\right]\right\} \\
- & {\left[\left(\zeta-\zeta_{3,1}^{\prime \prime}\right)^{-1}-\left(\zeta-\zeta_{3,2}^{\prime \prime}\right)^{-1}\right]\left\{\ln A_{3}(\zeta, 1-\zeta)-\ln B_{3}(\zeta, 1-\zeta)\right\} } \\
& -\left[\left(\zeta-\zeta_{1,1}\right)^{-1}-\left(\zeta-\zeta_{1,2}\right)^{-1}\right]\left\{\ln A_{1}(0, \zeta)-\ln \left[(1-\zeta) A_{1}(0, \zeta)+B_{1}(0, \zeta)\right]\right\} \\
& -\left[\left(\zeta-\zeta_{2,1}\right)^{-1}-\left(\zeta-\zeta_{2,2}\right)^{-1}\right]\left\{\ln A_{2}(0, \zeta)-\ln \left[\zeta A_{2}(0, \zeta)+B_{2}(0, \zeta)\right]\right\} \\
& \left.-\left[\left(\zeta-\zeta_{3,1}\right)^{-1}-\left(\zeta-\zeta_{3,2}\right)^{-1}\right]\left\{\ln A_{3}(0, \zeta)-\ln B_{3}(0, \zeta)\right\}\right\} .
\end{aligned}
$$

Finally, we perform the projective transformation,

$$
x=\frac{\zeta}{1-\zeta},
$$

with

$$
x_{i, j}^{\prime}=\frac{\zeta_{i, j}^{\prime}}{1-\zeta_{i, j}^{\prime}}=-\frac{z_{i, j}}{z_{i, j}+\beta_{i}}, \quad x_{i, j}^{\prime \prime}=\frac{\zeta_{i, j}^{\prime \prime}}{1-\zeta_{i, j}^{\prime \prime}}=\frac{1-z_{i, j}}{\beta_{i}+z_{i, j}}, \quad x_{i, j}=\frac{\zeta_{i, j}}{1-\zeta_{i, j}}=\frac{z_{i, j}}{1-z_{i, j}},
$$

leading to

$$
\begin{aligned}
D_{0}=\frac{1}{\sqrt{\operatorname{det} Y}} & \int_{0}^{\infty} \mathrm{d} x\left\{\left[\left(x-x_{1,1}^{\prime}\right)^{-1}-\left(x-x_{1,2}^{\prime}\right)^{-1}\right] l_{31}(x)\right. \\
+ & {\left[\left(x-x_{2,1}^{\prime}\right)^{-1}-\left(x-x_{2,2}^{\prime}\right)^{-1}\right] l_{21}(x) } \\
& +\left[\left(x-x_{3,1}^{\prime}\right)^{-1}-\left(x-x_{3,2}^{\prime}\right)^{-1}\right] l_{01}(x) \\
& -\left[\left(x-x_{1,1}^{\prime \prime}\right)^{-1}-\left(x-x_{1,2}^{\prime \prime}\right)^{-1}\right] l_{01}(x) \\
& -\left[\left(x-x_{2,1}^{\prime \prime}\right)^{-1}-\left(x-x_{2,2}^{\prime \prime}\right)^{-1}\right] l_{31}(x) \\
& -\left[\left(x-x_{3,1}^{\prime \prime}\right)^{-1}-\left(x-x_{3,2}^{\prime \prime}\right)^{-1}\right] l_{21}(x) \\
& -\left[\left(x-x_{1,1}\right)^{-1}-\left(x-x_{1,2}\right)^{-1}\right] l_{30}(x) \\
& -\left[\left(x-x_{2,1}\right)^{-1}-\left(x-x_{2,2}\right)^{-1}\right] l_{23}(x) \\
& \left.-\left[\left(x-x_{3,1}\right)^{-1}-\left(x-x_{3,2}\right)^{-1}\right] l_{02}(x)\right\},
\end{aligned}
$$


where we made use of the generic functions

$$
\begin{aligned}
l_{i j}(x) & =\ln (1+x)+\ln \left(M_{i}^{2}+M_{j}^{2} x\right)-\ln \left(m_{i}^{2}+Y_{i j} x+m_{j}^{2} x^{2}\right) \\
& =\ln M_{i}^{2}-\ln m_{i}^{2}+\ln (1+x)+\ln \left(1+\frac{M_{j}^{2}}{M_{i}^{2}} x\right)-\ln \left(1+r_{i j, 1} x\right)-\ln \left(1+r_{i j, 2} x\right),
\end{aligned}
$$

which depend on the constants

$$
\begin{aligned}
& M^{2}=-(1+\alpha) m_{0}^{2}+\alpha m_{2}^{2}+m_{3}^{2}, \\
& M_{0}^{2}=M^{2}-p_{30}^{2}-\alpha p_{20}^{2}, \\
& M_{1}^{2}=M^{2}+(1+\alpha) p_{10}^{2}-\alpha p_{21}^{2}-p_{31}^{2}, \\
& M_{2}^{2}=M^{2}+(1+\alpha) p_{20}^{2}-p_{32}^{2}, \\
& M_{3}^{2}=M^{2}+(1+\alpha) p_{30}^{2}-\alpha p_{32}^{2} .
\end{aligned}
$$

In the second relation of (3.35) we use the variables $r_{i j, k}$ as defined in (2.5). The validity of this relation for all real positive $x$ becomes obvious after realizing that it is trivially fulfilled for $x=0$ and that none of the arguments of the logarithms crosses a branch cut. Note that the freedom to add an infinitesimally small negative imaginary part to each mass $m_{i}^{2}$ should be exploited to render the imaginary part of $M^{2}$ (and thus of all $M_{i}^{2}$ ) non-zero. This point is important in the cases when some masses $m_{i}$ are equal, so that the imaginary parts entering $M^{2}$ might compensate, which should be avoided. It should also be realized that the contributions involving the variables $x_{i, j}^{\prime}$ or $x_{i, j}^{\prime \prime}$ drop out in the special cases $\beta_{i}=0$ or $\beta_{i}=-1$, respectively.

The integral representation (3.34), together with (3.35), is well suited to perform the analytical continuation from $\alpha>0$ and $\beta_{i}>0$ to any real values of $\alpha$ and $\beta_{i}$. During this continuation the masses $m_{i}$ are kept fixed. We first vary the squared momenta $p_{10}^{2}, p_{21}^{2}$, and $p_{31}^{2}$ to reach the domain where one or more $\beta_{i} \leq 0$, while keeping $p_{30}^{2}, p_{20}^{2}$, and $p_{32}^{2}$ (and thus also $\alpha, M^{2}, M_{0}^{2}, M_{2}^{2}$, and $M_{3}^{2}$ ) fixed. In this procedure, none of the arguments in the logarithms of the relevant $l_{i j}(x)$ crosses a branch cut. Moreover, it does not matter whether one of the zeroes $x_{i, j}, x_{i, j}^{\prime}, x_{i, j}^{\prime \prime}$ runs over the path of the $x$-integration, which is the positive real axes, or not, because the residues at the poles at $x_{i, j}, x_{i, j}^{\prime}, x_{i, j}^{\prime \prime}$ vanish as explained above. This shows that the continuation to $\beta_{i} \leq 0$ is trivial, i.e. that (3.34) is correct without modification. The only complication in the continuation to $\alpha \leq 0$ stems from the fact that the imaginary part of $M^{2}$ (and thus of all $M_{i}^{2}$ ) can change sign. This sign change can lead to a discontinuity of $\pm 2 \pi \mathrm{i}$ in the $\ln M_{i}^{2}$ term in $l_{i j}(x)$. However, these possible constant terms in $l_{i j}(x)$ compensate each other within the sum in (3.34), because after the $x$-integration they are proportional to

$$
\ln \left(-x_{i, j}^{\prime}\right)-\ln \left(-x_{i, j}^{\prime \prime}\right)-\ln \left(-x_{i, j}\right)=0 .
$$

This relation holds for all real values of $\beta_{i}$. Again no extra terms arise if any of the $x_{i, j}$, $x_{i, j}^{\prime}, x_{i, j}^{\prime \prime}$ runs over the integration axis because of the vanishing residues at these poles. In summary, (3.34) is valid without modification for all real values of $\alpha$ and $\beta_{i}$.

The $x$-integration in (3.34) can be easily carried out. Using the auxiliary integral (B.1) of Ref. [22], the final result reads

$$
D_{0}=\frac{1}{\sqrt{\operatorname{det} Y}} \sum_{k=1}^{2}(-1)^{k+1}\left\{L_{31}\left(x_{1, k}^{\prime}\right)+L_{21}\left(x_{2, k}^{\prime}\right)+L_{01}\left(x_{3, k}^{\prime}\right)-L_{01}\left(x_{1, k}^{\prime \prime}\right)-L_{31}\left(x_{2, k}^{\prime \prime}\right)\right.
$$




$$
\left.-L_{21}\left(x_{3, k}^{\prime \prime}\right)-L_{30}\left(x_{1, k}\right)-L_{23}\left(x_{2, k}\right)-L_{02}\left(x_{3, k}\right)\right\}
$$

with the generic function

$$
\begin{aligned}
L_{i j}(x)= & -\left[\ln M_{i}^{2}-\ln m_{i}^{2}\right] \ln (-x)+\operatorname{Li}_{2}(1+x) \\
& +\mathcal{L} i_{2}\left(\frac{M_{j}^{2}}{M_{i}^{2}},-x\right)-\mathcal{L} i_{2}\left(r_{i j, 1},-x\right)-\mathcal{L} i_{2}\left(r_{i j, 2},-x\right) .
\end{aligned}
$$

If $M_{i}^{2} \rightarrow 0$ the function $L_{i j}(x)$ reduces to

$$
\begin{aligned}
L_{i j}(x) \underset{M_{i}^{2} \rightarrow 0}{\longrightarrow}- & {\left[\ln M_{j}^{2}-\ln m_{i}^{2}\right] \ln (-x)-\frac{1}{2} \ln ^{2}(-x)+\operatorname{Li}_{2}(1+x) } \\
& -\mathcal{L} i_{2}\left(r_{i j, 1},-x\right)-\mathcal{L} i_{2}\left(r_{i j, 2},-x\right)+\text { const. }
\end{aligned}
$$

where the $x$-independent term indicated as "const." drops out in the difference $\sum_{k=1}^{2}(-1)^{k+1} L_{i j}\left(x_{k}\right)$. The final result (3.38) for the $D_{0}$ function with non-vanishing complex masses $m_{i}$ contains 72 dilogarithms of complex arguments.

\subsection{4-point integral for general complex masses - method of Ref. [22]}

Following the method of Ref. [22], we first derive a result for the 4-point function involving 16 dilogarithms that is valid for complex masses if one of the external momenta squared vanishes. Then we use an identity to express the general 4-point function in terms of two 4-point functions of this kind.

\subsubsection{General massive 4-point integral for one vanishing external momentum squared}

We start by deriving a result for the regular scalar 4-point function with four internal real masses and two space-like or light-like momenta. Upon analytic continuation in the masses and one of the momentum squares we generalise it to the case of complex masses and five arbitrary real invariants, while the sixth invariant has to fulfil certain conditions, which hold in particular if this invariant vanishes. We follow the methods of Ref. [22].

In the following we need the polynomials

$$
Q\left(x_{0}, x_{1}, x_{2}, x_{3}\right)=\left[-\frac{1}{r_{20}} \frac{\partial}{\partial x_{0}}+\frac{\partial}{\partial x_{2}}\right] P\left(x_{0}, x_{1}, x_{2}, x_{3}\right)
$$

and

$$
\bar{Q}\left(x_{0}, x_{1}, x_{2}, x_{3}\right)=\left[\frac{\partial}{\partial x_{1}}-\frac{1}{r_{13}} \frac{\partial}{\partial x_{3}}\right] P\left(x_{0}, x_{1}, x_{2}, x_{3}\right),
$$

where $r_{20}$ and $r_{13}$ are each one of the two variables defined in the corresponding equation (2.5).

We start from the representation (3.1) and assume that all masses are real and that the quantities $r_{20}$ and $r_{13}$ are real and positive, which is the case if the momenta $p_{20}$ and $p_{31}$ are space-like or light-like. We apply the projective transformation

$$
\left(\begin{array}{l}
x_{1} \\
x_{2} \\
x_{3}
\end{array}\right)=\frac{1}{1+x+\left(1-1 / r_{13}\right) y+\left(1-1 / r_{20}\right) z}\left(\begin{array}{c}
y \\
z \\
x-y / r_{13}
\end{array}\right)
$$


with the Jacobian

$$
\frac{\partial\left(x_{1}, x_{2}, x_{3}\right)}{\partial(x, y, z)}=\frac{1}{\left[1+x+\left(1-1 / r_{13}\right) y+\left(1-1 / r_{20}\right) z\right]^{4}}
$$

in order to linearise $P\left(1-x_{1}-x_{2}-x_{3}, x_{1}, x_{2}, x_{3}\right)$ simultaneously in two variables of integration. The transformation of the integration region is determined by the inverse

$$
\left(\begin{array}{l}
x \\
y \\
z
\end{array}\right)=\frac{1}{1-x_{1}-\left(1-1 / r_{20}\right) x_{2}-x_{3}}\left(\begin{array}{c}
x_{3}+x_{1} / r_{13} \\
x_{1} \\
x_{2}
\end{array}\right) .
$$

This yields

$$
D_{0}=\int_{0}^{\infty} \mathrm{d} x \int_{0}^{x r_{13}} \mathrm{~d} y \int_{0}^{r_{20}} \mathrm{~d} z \frac{1}{\left[P\left(1-z / r_{20}, y, z, x-y / r_{13}\right)-\mathrm{i} \varepsilon\right]^{2}},
$$

where $P$ is linear in both $y$ and $z$. Note that we have included an explicit infinitesimal imaginary part $-\mathrm{i} \varepsilon$ consistent with the infinitesimal imaginary part of the masses. However, for later use the masses are still assumed to have an infinitesimal imaginary part which is negligible compared to $i \varepsilon$. Performing the first integration and subsequently combining the resulting terms over a common denominator results in

$$
\begin{aligned}
D_{0}= & \int_{0}^{\infty} \mathrm{d} x \int_{0}^{x r_{13}} \mathrm{~d} y \frac{1}{Q\left(1, y, 0, x-y / r_{13}\right)} \\
& \times\left(\frac{1}{P\left(1, y, 0, x-y / r_{13}\right)-\mathrm{i} \varepsilon}-\frac{1}{P\left(0, y, r_{20}, x-y / r_{13}\right)-\mathrm{i} \varepsilon}\right) \\
= & r_{20} \int_{0}^{\infty} \mathrm{d} x \int_{0}^{x r_{13}} \mathrm{~d} y \frac{1}{P\left(1, y, 0, x-y / r_{13}\right)-\mathrm{i} \varepsilon} \frac{1}{P\left(0, y, r_{20}, x-y / r_{13}\right)-\mathrm{i} \varepsilon} .
\end{aligned}
$$

By means of a partial-fraction decomposition the second integration can be done as well,

$$
\begin{gathered}
D_{0}=\int_{0}^{\infty} \mathrm{d} x \frac{1}{R(x)} \int_{0}^{x r_{13}} \mathrm{~d} y\left(\frac{\bar{Q}(1,0,0, x)}{P\left(1, y, 0, x-y / r_{13}\right)-\mathrm{i} \varepsilon}-\frac{\bar{Q}\left(0,0, r_{20}, x\right)}{P\left(0, y, r_{20}, x-y / r_{13}\right)-\mathrm{i} \varepsilon}\right) \\
=\int_{0}^{\infty} \mathrm{d} x \frac{1}{R(x)}\left(\ln \left[P\left(0,0, r_{20}, x\right)-\mathrm{i} \varepsilon\right]-\ln [P(1,0,0, x)-\mathrm{i} \varepsilon]\right. \\
\left.\quad-\ln \left[P\left(0, x r_{13}, r_{20}, 0\right)-\mathrm{i} \varepsilon\right]+\ln \left[P\left(1, x r_{13}, 0,0\right)-\mathrm{i} \varepsilon\right]\right),
\end{gathered}
$$

where the $y$-independent function $R(x)$ is defined as

$$
\begin{aligned}
R(x)= & \frac{1}{r_{20}}\left[\bar{Q}(1,0,0, x)\left[P\left(0, y, r_{20}, x-y / r_{13}\right)-\mathrm{i} \varepsilon\right]\right. \\
& \left.-\bar{Q}\left(0,0, r_{20}, x\right)\left[P\left(1, y, 0, x-y / r_{13}\right)-\mathrm{i} \varepsilon\right]\right] \\
= & a x^{2}+b x+c+\mathrm{i} \varepsilon d=a\left(x-x_{1}\right)\left(x-x_{2}\right)
\end{aligned}
$$

with

$$
\begin{aligned}
a & =m_{1}^{2} r_{13}\left(Y_{23}-Y_{03} / r_{20}\right)-m_{3}^{2}\left(Y_{12}-Y_{01} / r_{20}\right), \\
b & =\left(m_{2}^{2} r_{20}-m_{0}^{2} / r_{20}\right)\left(m_{1}^{2} r_{13}-m_{3}^{2} / r_{13}\right)+Y_{01} Y_{23}-Y_{03} Y_{12}, \\
c & =m_{2}^{2} r_{20}\left(Y_{01}-Y_{03} / r_{13}\right)-m_{0}^{2}\left(Y_{12}-Y_{23} / r_{13}\right), \\
d & =Y_{12}-Y_{01} / r_{20}-Y_{23} / r_{13}+Y_{03} /\left(r_{20} r_{13}\right) .
\end{aligned}
$$


The discriminant of the quadratic form reads

$$
b^{2}-4 a c=\operatorname{det} Y
$$

where $Y$ is the modified Cayley matrix defined in (2.4), and the roots $x_{1,2}$ are given by

$$
x_{1,2}=\frac{1}{2 a}(-b \pm \sqrt{\operatorname{det} Y}) \mp \frac{\mathrm{i} \varepsilon d}{\sqrt{\operatorname{det} Y}} .
$$

Note that the infinitesimal imaginary part of the variables $x_{1,2}$ results only form the $\mathrm{i} \varepsilon d$ term in (3.49), i.e. real mass parameters enter the calculation of $x_{1,2}$ via (3.50) as truly real quantities without infinitesimal imaginary parts.

A further partial-fraction decomposition yields an integral representation valid for real and positive $r_{20}, r_{13}$ and real masses

$$
\begin{aligned}
& a\left(x_{1}-x_{2}\right) D_{0}=\int_{0}^{\infty} \mathrm{d} x\left(\frac{1}{x-x_{1}}-\frac{1}{x-x_{2}}\right) \\
& \quad \times\left\{\ln \left(\frac{P\left(0,0,1, x / r_{20}\right)-\mathrm{i} \varepsilon / r_{20}^{2}}{P(1,0,0, x)-\mathrm{i} \varepsilon}\right)-\ln \left(\frac{P\left(0, x r_{13} / r_{20}, 1,0\right)-\mathrm{i} \varepsilon / r_{20}^{2}}{P\left(1, x r_{13}, 0,0\right)-\mathrm{i} \varepsilon}\right)\right\} .
\end{aligned}
$$

The following algebraic relations with arbitrary $y$ and $z$ hold for $R(x)$ and prove useful for the analytic continuation

$$
\begin{aligned}
R(x)=\{ & {\left[P\left(1-z / r_{20}, 0, z, x\right)-\mathrm{i} \varepsilon\right]\left[P\left(1, y, 0, x-y / r_{13}\right)-\mathrm{i} \varepsilon\right] } \\
& \left.-[P(1,0,0, x)-\mathrm{i} \varepsilon]\left[P\left(1-z / r_{20}, y, z, x-y / r_{13}\right)-\mathrm{i} \varepsilon\right]\right\} /(y z) \\
=- & \left\{\bar{Q}\left(1-z / r_{20}, 0, z, x\right)\left[P\left(1, y, 0, x-y / r_{13}\right)-\mathrm{i} \varepsilon\right]\right. \\
& \left.-\bar{Q}(1,0,0, x)\left[P\left(1-z / r_{20}, y, z, x-y / r_{13}\right)-\mathrm{i} \varepsilon\right]\right\} / z \\
=\{ & (1,0,0, x)\left[P\left(1, y, 0, x-y / r_{13}\right)-\mathrm{i} \varepsilon\right] \\
& \left.-Q\left(1, y, 0, x-y / r_{13}\right)[P(1,0,0, x)-\mathrm{i} \varepsilon]\right\} / y \\
=\{ & \bar{Q}\left(1-z / r_{20}, 0, z, x\right) Q\left(1, y, 0, x-y / r_{13}\right) \\
& \left.-d\left[P\left(1-z / r_{20}, y, z, x-y / r_{13}\right)-\mathrm{i} \varepsilon\right]\right\} .
\end{aligned}
$$

In order to perform the analytic continuation, we rewrite (3.53) as

$$
\begin{aligned}
a\left(x_{1}-x_{2}\right) D_{0}= & \int_{0}^{\infty} \mathrm{d} x\left(\frac{1}{x-x_{1}}-\frac{1}{x-x_{2}}\right) \ln \left(\frac{P\left(0,0,1, x / r_{20}\right)-\mathrm{i} \varepsilon / r_{20}^{2}}{P(1,0,0, x)-\mathrm{i} \varepsilon}\right) \\
& -\int_{0}^{\infty / r_{13}} \mathrm{~d} x\left(\frac{1}{x-x_{1}}-\frac{1}{x-x_{2}}\right) \ln \left(\frac{P\left(0, x r_{13} / r_{20}, 1,0\right)-\mathrm{i} \varepsilon / r_{20}^{2}}{P\left(1, x r_{13}, 0,0\right)-\mathrm{i} \varepsilon}\right) \\
& +\sum_{k=1}^{2}(-1)^{k} \ln \left(\frac{P\left(0,0,1, x_{k} / r_{20}\right)-\mathrm{i} \varepsilon / r_{20}^{2}}{P\left(1,0,0, x_{k}\right)-\mathrm{i} \varepsilon}\right) \oint_{\left(0, \infty, \infty / r_{13}\right)} \frac{\mathrm{d} x}{x-x_{k}},
\end{aligned}
$$




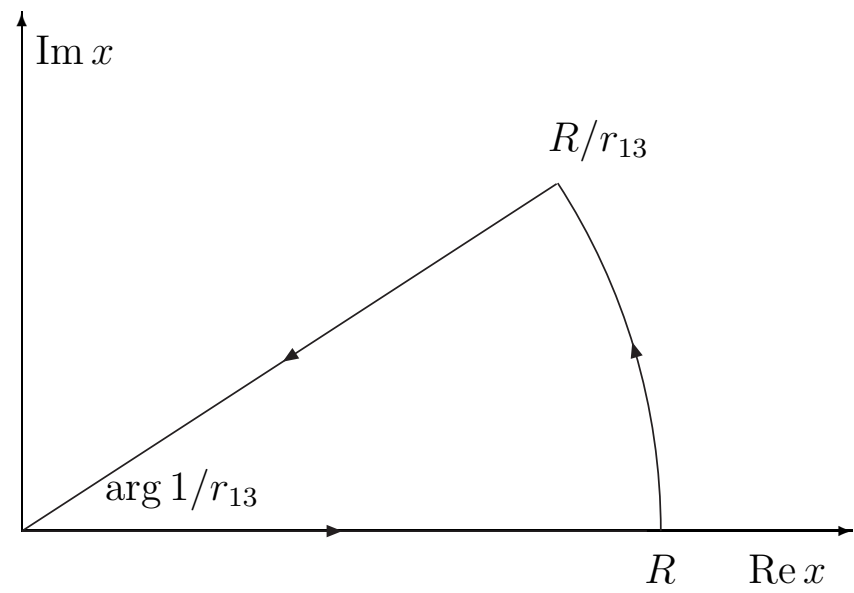

Figure 1: The contour $\left(0, R, R / r_{13}\right)$ in the complex $x$-plane

where the contour of the integrals in the last line results from the one shown in Figure 1 in the limit $R \rightarrow \infty$. Equation (3.58) obviously coincides with (3.53) for real masses and $r_{13}, r_{20}>0$. Now we allow for complex masses and arbitrary $p_{31}^{2}$, leading to complex $r_{13}$, while $r_{20}$ is still required to be real and positive (which entails some restrictions on $p_{20}^{2}$ and/or the masses $m_{0}^{2}$ and $m_{2}^{2}$ ). We note that, in particular, for $p_{20}^{2}=0$ we can choose $r_{20}=1$, so that our result holds. We here allow also squared masses with negative real parts, but always with negative imaginary parts, a case for which (2.1) can be directly analytically continued. Equation (3.58) yields the correct analytical continuation, since:

- No cut is crossed in the logarithms of the first two integrals of (3.58), since all polynomials $P$ have negative imaginary parts. Note that $x, x / r_{20}$ in the first integral and $x r_{13}, x r_{13} / r_{20}$ in the second integral are real.

- If one of the poles $x_{1,2}$ crosses the integration contour in one of the first two integrals, it also moves into the closed contour $\left(0, \infty, \infty / r_{13}\right)$. The residues add up to zero owing to (3.54) where one has to set $(y, z)=\left(x r_{13}, r_{20}\right)$. For real and positive $x_{1,2}$ the explicit imaginary parts in (3.52) and (3.58) proportional to i $\varepsilon$ are crucial for this cancellation, i.e. both have to be taken into account consistently (with the same numerical size of $\varepsilon$ ).

- Finally, we have to show that there are no further discontinuities in the last term of (3.58), apart from the required ones resulting from the possible movement of $x_{1,2}$ inside the integration contour. If $x_{k}$ is outside the contour, nothing is to show, because the term is zero. If $x_{k}$ is within the closed contour of the integral in the last line of (3.58), it can be written as $x_{k}=u+v / r_{13}$ with real and positive $u$ and $v$. Using (3.54) for $x=x_{k}, y=v$ and $z=r_{20}$, we find

$$
\frac{P\left(0,0,1, x_{k} / r_{20}\right)-\mathrm{i} \varepsilon / r_{20}^{2}}{P\left(1,0,0, x_{k}\right)-\mathrm{i} \varepsilon}=\frac{P\left(0, v / r_{20}, 1, u / r_{20}\right)-\mathrm{i} \varepsilon / r_{20}^{2}}{P(1, v, 0, u)-\mathrm{i} \varepsilon} .
$$

For real and positive $r_{20}$, both $P\left(0, v / r_{20}, 1, u / r_{20}\right)-$ i $\varepsilon$ and $P(1, v, 0, u)-$ i $\varepsilon$ have a negative imaginary part and the ratio does never cross the cut.

If the ratio approaches the cut of the logarithm, its imaginary part can be determined from (3.59) as long as $r_{13}$ has a finite imaginary part (otherwise $v$ is not defined). 
If $r_{13}$ is real, the last line of (3.58) contributes only if $r_{13}$ is negative. In this case the imaginary part of the ratio can be determined using (3.55) via

$$
\begin{aligned}
\frac{P\left(0,0,1, x_{k} / r_{20}\right)-\mathrm{i} \varepsilon / r_{20}^{2}}{P\left(1,0,0, x_{k}\right)-\mathrm{i} \varepsilon} & =\frac{\bar{Q}\left(0,0, r_{20}, x_{k}\right)}{r_{20}^{2} \bar{Q}\left(1,0,0, x_{k}\right)} \\
& =\frac{r_{20}\left(Y_{12}-Y_{23} / r_{13}\right)+\left(m_{1}^{2} r_{13}-m_{3}^{2} / r_{13}\right) x_{k}}{r_{20}^{2}\left[Y_{01}-Y_{03} / r_{13}+\left(m_{1}^{2} r_{13}-m_{3}^{2} / r_{13}\right) x_{k}\right]}
\end{aligned}
$$

taking into account only the infinitesimal imaginary part of $x_{k}$ resulting from i $\varepsilon$. Obviously, the imaginary parts of the numerator and denominator of the last expression have the same imaginary part (from $x_{k}$ ) near the real axis, so that the ratio cannot cross the cut.

Consequently, (3.58) provides a correct analytical continuation. We finally reemphasize the treatment of infinitesimal negative imaginary parts attributed to real mass squares. While these infinitesimal imaginary parts are made explicit in the calculation of $x_{k}$ by the i $\varepsilon$ terms (and whenever i $\varepsilon$ appears), the infinitesimal imaginary parts of the variables $r_{i j, k}$ of (2.5) are implicitly determined from the ones in the squared masses (or equivalently from $\bar{p}_{i j}^{2}$ ) and are negligible compared to i $\varepsilon$, i.e., the infinitesimal imaginary part of $x_{k} r_{13}$ is solely determined by the infinitesimal imaginary part of $x_{k}$.

Using the auxiliary integral (B.1) of Ref. [22, (3.58) can be evaluated to

$$
\begin{aligned}
a\left(x_{1}-\right. & \left.x_{2}\right) D_{0}\left(p_{1}, p_{2}, p_{3}, m_{0}^{2}, m_{1}^{2}, m_{2}^{2}, m_{3}^{2}\right)= \\
= & \sum_{k=1}^{2}(-1)^{k+1}\left\{\sum _ { l = 1 } ^ { 2 } \left[\mathcal{L} i_{2}\left(-x_{k}, \frac{r_{23, l}}{r_{20}}\right)-\mathcal{L} i_{2}\left(-x_{k}, r_{03, l}\right)\right.\right. \\
& \left.\quad-\mathcal{L} i_{2}\left(-x_{k} r_{13}, \frac{r_{21, l}}{r_{20}}\right)+\mathcal{L} i_{2}\left(-x_{k} r_{13}, r_{01, l}\right)\right] \\
& \left.\quad \eta\left(-x_{k}, r_{13}\right)\left[\ln \left(\frac{P\left(0,0,1, x_{k} / r_{20}\right)-\mathrm{i} \varepsilon / r_{20}^{2}}{P\left(1,0,0, x_{k}\right)-\mathrm{i} \varepsilon}\right)-\ln \left(\frac{m_{2}^{2}}{m_{0}^{2}}\right)\right]\right\} .
\end{aligned}
$$

Note that also for negative squared masses, $r_{i j}$ do never cross the negative real axis as long as the squared masses have negative (infinitesimal) imaginary parts. The result (3.61) is in particular valid for $p_{20}^{2}=0$, where $r_{20}=1$, and arbitrary real values for the other invariants and complex squared masses with negative imaginary parts (and positive or negative real parts). Owing to the permutation symmetry of the arguments, it can be used if any external squared momentum vanishes, $p_{i j}^{2}=0$.

\subsubsection{Result for general massive 4-point function via propagator identity}

The result of the previous subsection can be used to derive a second result for the general scalar 4-point integral $D_{0}$ with complex internal masses. To this end, we employ a propagator identity of Ref. [18] to express the general $D_{0}$ in terms of two 4-point functions with one vanishing external momentum squared. For the latter functions the result (3.61) is applicable. Thus, we end up with a result in terms of 32 dilogarithms for the general $D_{0}$ function with complex masses. 
The propagator identity (2.2) of Ref. [18] allows to express the product of two propagators in terms of a sum of two products of two propagators,

$$
\frac{1}{\left(q+p_{1}\right)^{2}-m_{1}^{2}} \frac{1}{\left(q+p_{3}\right)^{2}-m_{3}^{2}}=\frac{1}{(q+p)^{2}-M^{2}}\left[\frac{1-\gamma}{\left(q+p_{1}\right)^{2}-m_{1}^{2}}+\frac{\gamma}{\left(q+p_{3}\right)^{2}-m_{3}^{2}}\right],
$$

where $\gamma$ is arbitrary and

$$
\begin{aligned}
p & =\gamma p_{1}+(1-\gamma) p_{3}, \\
M^{2} & =p^{2}+\gamma\left(m_{1}^{2}-p_{1}^{2}\right)+(1-\gamma)\left(m_{3}^{2}-p_{3}^{2}\right) .
\end{aligned}
$$

Fixing $\gamma$ in such a way that (recall $p_{1}^{2}=p_{10}^{2}$ and $p_{3}^{2}=p_{30}^{2}$ )

$$
p^{2}=\gamma^{2} p_{31}^{2}+\gamma\left(p_{10}^{2}-p_{31}^{2}-p_{30}^{2}\right)+p_{30}^{2}=0
$$

and inserting the identity (3.62) into (2.1), we can express the general scalar 4-point function as

$$
\begin{aligned}
D_{0}\left(p_{10}^{2},\right. & \left.p_{21}^{2}, p_{32}^{2}, p_{30}^{2}, p_{20}^{2}, p_{31}^{2}, m_{0}^{2}, m_{1}^{2}, m_{2}^{2}, m_{3}^{2}\right) \\
= & \gamma D_{0}\left(0,\left(p_{2}-p\right)^{2}, p_{32}^{2}, p_{30}^{2}, p_{20}^{2}, \gamma^{2} p_{31}^{2}, m_{0}^{2}, M^{2}, m_{2}^{2}, m_{3}^{2}\right) \\
& +(1-\gamma) D_{0}\left(p_{10}^{2}, p_{21}^{2},\left(p_{2}-p\right)^{2}, 0, p_{20}^{2},(1-\gamma)^{2} p_{31}^{2}, m_{0}^{2}, m_{1}^{2}, m_{2}^{2}, M^{2}\right) \\
= & \gamma D_{0}\left(p_{30}^{2}, \gamma^{2} p_{31}^{2},\left(p_{2}-p\right)^{2}, p_{20}^{2}, 0, p_{32}^{2}, m_{0}^{2}, m_{3}^{2}, M^{2}, m_{2}^{2}\right) \\
& \quad+(1-\gamma) D_{0}\left(p_{10}^{2},(1-\gamma)^{2} p_{31}^{2},\left(p_{2}-p\right)^{2}, p_{20}^{2}, 0, p_{21}^{2}, m_{0}^{2}, m_{1}^{2}, M^{2}, m_{2}^{2}\right),
\end{aligned}
$$

where

$$
\left(p_{2}-p\right)^{2}=\gamma\left(p_{21}^{2}-p_{10}^{2}\right)+(1-\gamma)\left(p_{32}^{2}-p_{30}^{2}\right),
$$

and we used the symmetry of $D_{0}$ mentioned after (2.4) to arrange the arguments such that the 5 th momentum squared vanishes.

As mentioned above, $\gamma$ [which corresponds to $-1 / \beta_{1}$ of (3.16)] is real for all momentum configurations appearing in $1 \rightarrow n$ decays or $2 \rightarrow n$ scattering processes. For such processes, at least one of the momenta $p_{31}, p_{30}$, and $p_{10}$ is time-like. Since the interchange $p_{30} \leftrightarrow p_{10}$ replaces $\gamma$ by $1-\gamma$ and $p_{31} \leftrightarrow p_{30}$ transforms $\gamma$ into $1 / \gamma$, the momenta can always be permuted such that

$$
0 \leq \gamma \leq 1
$$

Then in (3.65) the squared masses have negative imaginary parts (while the real part of $M^{2}$ may become negative), and the result (3.61) holds.

To be specific we select a suitable permutation of the arguments of $D_{0}$ as follows:

- If there is no $t$-channel-like squared momentum among the arguments of $D_{0}$, the centre-of-mass energy squared $s$ must be one of the arguments, and we can choose $p_{31}^{2}=s$ and

$$
\gamma=\frac{1}{2 p_{31}^{2}}\left[p_{31}^{2}+p_{30}^{2}-p_{10}^{2}+\sqrt{\left(p_{31}^{2}+p_{30}^{2}-p_{10}^{2}\right)^{2}-4 p_{31}^{2} p_{30}^{2}}\right]
$$

where $p_{10}$ can be any sum of outgoing momenta and $p_{10}=p_{30}-p_{31}$. The choice (3.68) fulfils (3.67) since $p_{31}^{2} \geq\left(\sqrt{p_{30}^{2}}+\sqrt{p_{10}^{2}}\right)^{2}$. 
- For one $t$-channel-like squared momentum $t$ we choose $p_{30}^{2}=t$. Since $p_{31}^{2} \geq 0$, $p_{10}^{2} \geq 0$ and $p_{30}^{2} \leq\left(\sqrt{p_{31}^{2}}-\sqrt{p_{10}^{2}}\right)^{2}$ for any choice of line 1 of the diagram, $\gamma$ defined by (3.68) fulfils (3.67) again.

- If there are two $t$-channel-like momentum invariants $t_{1}$ and $t_{2}$, we choose $p_{31}^{2}=t_{1}$ and $p_{10}^{2}=t_{2}$. Since $p_{30}^{2} \geq 0$,

$$
\gamma=\frac{1}{2 p_{31}^{2}}\left[p_{31}^{2}+p_{30}^{2}-p_{10}^{2}-\sqrt{\left(p_{31}^{2}+p_{30}^{2}-p_{10}^{2}\right)^{2}-4 p_{31}^{2} p_{30}^{2}}\right]
$$

fulfils (3.67).

In conclusion, (3.65) together with (3.61) and the appropriate permutation of momenta and choice of $\gamma$ according to (3.68) or (3.69) provides a result for the general scalar 4-point function with non-vanishing complex masses, valid for all scattering and decay processes.

\subsection{Regular 4-point function with one or more masses vanishing}

For vanishing masses the general results of the last two sections are not directly applicable. Instead of extracting the corresponding limits from the final results, it is more convenient to use the result (3.53) which is based on Ref. [ 22]. If certain variables diverge in the following formulas (e.g. for $a=0$ ), a well-defined result is obtained by permuting propagator lines of the diagram, as long as the integral is non-singular.

We first consider the case of one vanishing mass and derive a result for $m_{2}^{2}=0$ and $m_{0}^{2}$, $m_{1}^{2}, m_{3}^{2}$ non-zero and possibly complex with negative imaginary parts. It is appropriate to start by considering real masses and space-like or light-like momenta $p_{20}$ and $p_{31}$, so that $r_{20}$ and $r_{13}$ are real and positive. In order to make use of the result (3.53), we choose the solution for $r_{20}$ that satisfies

$$
\frac{1}{r_{20}} \underset{m_{2}^{2} \rightarrow 0}{\longrightarrow} 0, \quad m_{2}^{2} r_{20} \underset{m_{2}^{2} \rightarrow 0}{\longrightarrow} Y_{02}
$$

Then, we obtain

$$
\begin{aligned}
\frac{P\left(0,0,1, x / r_{20}\right)-\mathrm{i} \varepsilon / r_{20}^{2}}{P\left(0, x r_{13} / r_{20}, 1,0\right)-\mathrm{i} \varepsilon / r_{20}^{2}} \underset{m_{2}^{2} \rightarrow 0}{\longrightarrow} \frac{Q\left(0,0,1, x / r_{20}\right)}{Q\left(0, x r_{13} / r_{20}, 1,0\right)} \underset{m_{2}^{2} \rightarrow 0}{\underset{Q\left(1, x r_{13}, 0,0\right)}{Q}} \frac{Q(1,0,0, x)}{\underset{m_{2}^{2} \rightarrow 0}{\longrightarrow} \frac{Y_{02}+Y_{23} x}{Y_{02}+Y_{12} x r_{13}}}
\end{aligned}
$$

where the explicit i $\varepsilon$ terms on the very l.h.s. vanish for $m_{2}^{2} \rightarrow 0$ so that the infinitesimal imaginary parts are implicitly fixed by those of the internal masses contained in the $Q$ functions and $Y_{i j}$ variables. With these replacements, (3.53) can be written as

$$
\begin{aligned}
a\left(x_{1}-\right. & \left.x_{2}\right) D_{0}\left(p_{1}, p_{2}, p_{3}, m_{0}^{2}, m_{1}^{2}, 0, m_{3}^{2}\right) \\
= & \int_{0}^{\infty} \mathrm{d} x\left(\frac{1}{x-x_{1}}-\frac{1}{x-x_{2}}\right) \ln \left(\frac{Q(1,0,0, x)}{P(1,0,0, x)-\mathrm{i} \varepsilon}\right) \\
& \quad-\int_{0}^{\infty / r_{13}} \mathrm{~d} x\left(\frac{1}{x-x_{1}}-\frac{1}{x-x_{2}}\right) \ln \left(\frac{Q\left(1, x r_{13}, 0,0\right)}{P\left(1, x r_{13}, 0,0\right)-\mathrm{i} \varepsilon}\right)
\end{aligned}
$$




$$
\begin{aligned}
& +\sum_{k=1}^{2}(-1)^{k} \ln \left(\frac{Q\left(1,0,0, x_{k}\right)}{P\left(1,0,0, x_{k}\right)-\mathrm{i} \varepsilon}\right) \oint_{\left(0, \infty, \infty / r_{13}\right)} \frac{\mathrm{d} x}{x-x_{k}} \\
= & \int_{0}^{\infty} \mathrm{d} x\left(\frac{1}{x-x_{1}}-\frac{1}{x-x_{2}}\right) \ln \left(\frac{Y_{02}+Y_{23} x}{m_{0}^{2}+Y_{03} x+m_{3}^{2} x^{2}-\mathrm{i} \varepsilon}\right) \\
& -\int_{0}^{\infty / r_{13}} \mathrm{~d} x\left(\frac{1}{x-x_{1}}-\frac{1}{x-x_{2}}\right) \ln \left(\frac{Y_{02}+Y_{12} x r_{13}}{m_{0}^{2}+Y_{01} x r_{13}+m_{1}^{2}\left(x r_{13}\right)^{2}-\mathrm{i} \varepsilon}\right) \\
& +\sum_{k=1}^{2}(-1)^{k} \ln \left(\frac{Y_{02}+Y_{23} x_{k}}{m_{0}^{2}+Y_{03} x_{k}+m_{3}^{2} x_{k}^{2}-\mathrm{i} \varepsilon}\right) \oint_{\left(0, \infty, \infty / r_{13}\right)} \frac{\mathrm{d} x}{x-x_{k}},
\end{aligned}
$$

where again the contour of the last integral goes from zero to infinity on the positive real axis, along an arc at infinity to the direction of $1 / r_{13}$ and back to the origin (see Figure 1 for $R \rightarrow \infty)$. The quantities $x_{1,2}$ are determined from (3.49) with

$$
\begin{aligned}
a & =m_{1}^{2} r_{13} Y_{23}-m_{3}^{2} Y_{12}, \\
b & =Y_{02}\left(m_{1}^{2} r_{13}-m_{3}^{2} / r_{13}\right)+Y_{01} Y_{23}-Y_{03} Y_{12}, \\
c & =Y_{02}\left(Y_{01}-Y_{03} / r_{13}\right)-m_{0}^{2}\left(Y_{12}-Y_{23} / r_{13}\right), \\
d & =Y_{12}-Y_{23} / r_{13} .
\end{aligned}
$$

The result (3.72) is valid for real masses and real and positive $r_{13}$. The analytic continuation proceeds as for (3.58). We move the squared masses into the complex plane (with negative imaginary part) and $p_{31}^{2}$ to arbitrary real values. Thereby $r_{13}$ becomes complex and moves on the first Riemann sheet. Equation (3.72) yields the correct analytical continuation, since:

- The arguments of the logarithms in the first two integrals do not cross the cut, because numerator and denominator of the fractions both have negative imaginary parts (which in the numerator results from the masses).

- If one of the poles $x_{1,2}$ crosses the integration contour in one of the first two integrals, it also moves into the closed contour $\left(0, \infty, \infty / r_{13}\right)$. The residues add up to zero owing to (3.56) where one has to set $y=x r_{13}$. As stressed also above, for $x_{1,2}$ that are close to the positive real axis the explicit i $\varepsilon$ parts in (3.52) and (3.72) are crucial for this cancellation.

- If $x_{k}$ is within the closed contour of the integral in the last line, it can be written as $x_{k}=u+v / r_{13}$ with real and positive $u$ and $v$. Using (3.56) for $x=x_{k}$ and $y=v$, we find

$$
\begin{aligned}
\frac{P\left(1,0,0, x_{k}\right)-\mathrm{i} \varepsilon}{Q\left(1,0,0, x_{k}\right)} & =\frac{P(1, v, 0, u)-\mathrm{i} \varepsilon}{Q(1, v, 0, u)} \\
& =\frac{m_{0}^{2}+m_{1}^{2} v^{2}+m_{3}^{2} u^{2}+Y_{01} v+Y_{03} u+Y_{13} u v-\mathrm{i} \varepsilon}{Y_{02}+Y_{12} v+Y_{23} u}
\end{aligned}
$$

For squared masses with negative imaginary parts both $Q(1, v, 0, u)$ and $P(1, v, 0, u)-$ i $\varepsilon$ have a negative imaginary part and the ratio does not cross the cut. If the argument of the logarithm approaches the cut, its imaginary part can be determined 
from (3.74) as long as $\operatorname{Im} r_{13} \neq 0$ (otherwise $v$ is not defined). If the imaginary part of $r_{13}$ vanishes, the imaginary part of the ratio can be determined via [using (3.57)]

$$
\frac{P\left(1,0,0, x_{k}\right)-\mathrm{i} \varepsilon}{Q\left(1,0,0, x_{k}\right)}=\frac{\bar{Q}\left(1,0,0, x_{k}\right)}{d}=\frac{Y_{01}-Y_{03} / r_{13}+\left(m_{1}^{2} r_{13}-m_{3}^{2} / r_{13}\right) x_{k}}{Y_{12}-Y_{23} / r_{13}},
$$

taking into account only the infinitesimal imaginary part of $x_{k}$ resulting from i $\varepsilon$.

Employing the auxiliary integral (B.1) of Ref. [22], (3.72) can be evaluated to

$$
\begin{aligned}
a\left(x_{1}-\right. & \left.x_{2}\right) D_{0}\left(p_{1}, p_{2}, p_{3}, m_{0}^{2}, m_{1}^{2}, 0, m_{3}^{2}\right) \\
= & \sum_{k=1}^{2}(-1)^{k+1}\left\{\mathcal{L} i_{2}\left(-x_{k}, \frac{Y_{23}}{Y_{02}}\right)-\mathcal{L} i_{2}\left(-x_{k}, r_{03,1}\right)-\mathcal{L} i_{2}\left(-x_{k}, r_{03,2}\right)\right. \\
& -\mathcal{L} i_{2}\left(-x_{k} r_{13}, \frac{Y_{12}}{Y_{02}}\right)+\mathcal{L} i_{2}\left(-x_{k} r_{13}, r_{01,1}\right)+\mathcal{L} i_{2}\left(-x_{k} r_{13}, r_{01,2}\right) \\
& \left.+\eta\left(-x_{k}, r_{13}\right)\left[\ln \left(\frac{P\left(1,0,0, x_{k}\right)-\mathrm{i} \varepsilon}{Q\left(1,0,0, x_{k}\right)}\right)+\ln \left(\frac{Y_{02}}{m_{0}^{2}}\right)\right]\right\} .
\end{aligned}
$$

For $Y_{02}=m_{0}^{2}-p_{20}^{2}=0$ and $Y_{23} \neq 0$ this result can be used after exchanging indices 0 and 3 (in the sense of interchanging lines 0 and 3 in the diagram and performing the whole calculation with this assignment). For $Y_{02}=Y_{23}=0, Y_{02}=Y_{12}=0$, or $Y_{12}=Y_{23}=0$ the integral becomes soft singular; these cases are covered in the next section.

Next we consider the case of two vanishing masses and assume $m_{1}^{2}=m_{2}^{2}=0$, but $m_{0}^{2} \neq 0$ and $m_{3}^{2} \neq 0$. The other cases with two zero-masses can be obtained by obvious substitutions 2 We choose the roots such that

$$
\frac{1}{r_{13}} \underset{m_{1}^{2} \rightarrow 0}{\longrightarrow} 0, \quad m_{1}^{2} r_{13} \underset{m_{1}^{2} \rightarrow 0}{\longrightarrow} Y_{13}, \quad r_{01,1} \underset{m_{1}^{2} \rightarrow 0}{\longrightarrow} \frac{Y_{01}}{m_{0}^{2}}, \quad r_{13} r_{01,2} \underset{m_{1}^{2} \rightarrow 0}{\longrightarrow} \frac{Y_{13}}{Y_{01}},
$$

and obtain

$$
\begin{aligned}
& a\left(x_{1}-x_{2}\right) D_{0}\left(p_{1}, p_{2}, p_{3}, m_{0}^{2}, 0,0, m_{3}^{2}\right) \\
& =\sum_{k=1}^{2}(-1)^{k+1}\left\{\mathcal{L} i_{2}\left(-x_{k}, \frac{Y_{23}}{Y_{02}}\right)-\mathcal{L} i_{2}\left(-x_{k}, r_{03,1}\right)-\mathcal{L} i_{2}\left(-x_{k}, r_{03,2}\right)\right. \\
& \left.\quad+\mathcal{L} i_{2}\left(-x_{k}, \frac{Y_{13}}{Y_{01}}\right)-\ln \left(-x_{k}\right)\left[\ln \left(\frac{Y_{01}}{Y_{12}}\right)+\ln \left(\frac{Y_{02}}{m_{0}^{2}}\right)\right]\right\},
\end{aligned}
$$

for real positive $Y_{13}$, where the $x_{k}$ are determined from (3.49) with

$$
\begin{aligned}
a & =Y_{13} Y_{23}-m_{3}^{2} Y_{12}, & & b=Y_{02} Y_{13}+Y_{01} Y_{23}-Y_{03} Y_{12}, \\
c & =Y_{01} Y_{02}-m_{0}^{2} Y_{12}, & & d=Y_{12} .
\end{aligned}
$$

By going back to the corresponding integral representation (3.72) one can convince oneself that (3.78) is valid for general momenta and complex masses without modification. If

\footnotetext{
${ }^{2}$ This includes also the case with vanishing masses on opposite lines of the diagram because of the symmetry w.r.t. interchange of neighbouring lines, $D_{0}\left(p_{10}^{2}, p_{21}^{2}, p_{32}^{2}, p_{30}^{2}, p_{20}^{2}, p_{31}^{2}, m_{0}^{2}, m_{1}^{2}, m_{2}^{2}, m_{3}^{2}\right)=$ $D_{0}\left(p_{10}^{2}, p_{20}^{2}, p_{32}^{2}, p_{31}^{2}, p_{21}^{2}, p_{30}^{2}, m_{1}^{2}, m_{0}^{2}, m_{2}^{2}, m_{3}^{2}\right)$.
} 
$Y_{01}=0$ or $Y_{02}=0$ the result can be used after interchanging indices 0 and 3, as long as $Y_{23}$ and $Y_{13}$ do not vanish. For $Y_{01}=Y_{23}=0$ it can be written as

$$
\begin{aligned}
& a\left(x_{1}-x_{2}\right) D_{0}\left(p_{1}, p_{2}, p_{3}, m_{0}^{2}, 0,0, m_{3}^{2}\right) \\
& =\sum_{k=1}^{2}(-1)^{k+1}\left\{-\mathcal{L} i_{2}\left(-x_{k}, r_{03,1}\right)-\mathcal{L} i_{2}\left(-x_{k}, r_{03,2}\right)-\frac{1}{2} \ln ^{2}\left(-x_{k}\right)\right. \\
& \left.\quad-\ln \left(-x_{k}\right)\left[\ln \left(\frac{Y_{13}}{Y_{12}}\right)+\ln \left(\frac{Y_{02}}{m_{0}^{2}}\right)\right]\right\},
\end{aligned}
$$

which can also be used for $Y_{02}=0$ and $Y_{13}=0$ after interchanging indices 0 and 3. Other degenerate cases are soft or collinear singular (see Section 4).

For the case of three vanishing masses, consider $m_{1}^{2}=m_{2}^{2}=m_{3}^{2}=0$, but $m_{0}^{2} \neq 0$. The other cases can be obtained by obvious substitutions. Using

$$
r_{03,1} \underset{m_{3}^{2} \rightarrow 0}{\longrightarrow} \frac{Y_{03}}{m_{0}^{2}}, \quad r_{03,2} \underset{m_{3}^{2} \rightarrow 0}{\longrightarrow} 0,
$$

we obtain directly from (3.78) and (3.79)

$$
\begin{aligned}
a\left(x_{1}-\right. & \left.x_{2}\right) D_{0}\left(p_{1}, p_{2}, p_{3}, m_{0}^{2}, 0,0,0\right) \\
= & \sum_{k=1}^{2}(-1)^{k+1}\left\{\mathcal{L} i_{2}\left(-x_{k}, \frac{Y_{23}}{Y_{02}}\right)-\mathcal{L} i_{2}\left(-x_{k}, \frac{Y_{03}}{m_{0}^{2}}\right)\right. \\
& \left.\quad+\mathcal{L} i_{2}\left(-x_{k}, \frac{Y_{13}}{Y_{01}}\right)-\ln \left(-x_{k}\right)\left[\ln \left(\frac{Y_{01}}{Y_{12}}\right)+\ln \left(\frac{Y_{02}}{m_{0}^{2}}\right)\right]\right\}
\end{aligned}
$$

and

$$
\begin{aligned}
a & =Y_{13} Y_{23}, & b & =Y_{02} Y_{13}+Y_{01} Y_{23}-Y_{03} Y_{12}, \\
c & =Y_{01} Y_{02}-m_{0}^{2} Y_{12}, & d & =Y_{12} .
\end{aligned}
$$

Degenerate cases are obtained as for (3.78).

Finally for four vanishing masses, (3.82) and (3.83) simplify to

$$
\begin{aligned}
a\left(x_{1}-\right. & \left.x_{2}\right) D_{0}\left(p_{1}, p_{2}, p_{3}, 0,0,0,0\right) \\
= & \sum_{k=1}^{2}(-1)^{k+1}\left\{\mathcal{L} i_{2}\left(-x_{k}, \frac{Y_{23}}{Y_{02}}\right)-\mathcal{L} i_{2}\left(-\frac{1}{x_{k}}, \frac{Y_{01}}{Y_{13}}\right)\right. \\
& \left.\quad-\ln \left(-x_{k}\right)\left[\ln \left(\frac{Y_{13}}{Y_{12}}\right)+\ln \left(\frac{Y_{02}}{Y_{03}}\right)\right]\right\}
\end{aligned}
$$

and

$$
a=Y_{13} Y_{23}, \quad b=Y_{02} Y_{13}+Y_{01} Y_{23}-Y_{03} Y_{12}, \quad c=Y_{01} Y_{02}, \quad d=Y_{12} .
$$

Degenerate cases are mass singular. Different results for this case have been given in Refs. [19, 27]. 


\section{Singular 4-point integrals}

Whenever mass parameters $\lambda$ or $\lambda_{i}$ appear, they are understood as infinitesimal. If not all singularities are cured by mass parameters, dimensional regularization is applied. Thus, in the following all formulas are valid up to orders $\mathcal{O}(\lambda), \mathcal{O}\left(\lambda_{i}\right)$, and $\mathcal{O}(\epsilon)$. For convenience, we define the shorthand

$$
c_{\epsilon}=\Gamma(1+\epsilon)(4 \pi)^{\epsilon}
$$

and recall our graphical notation,

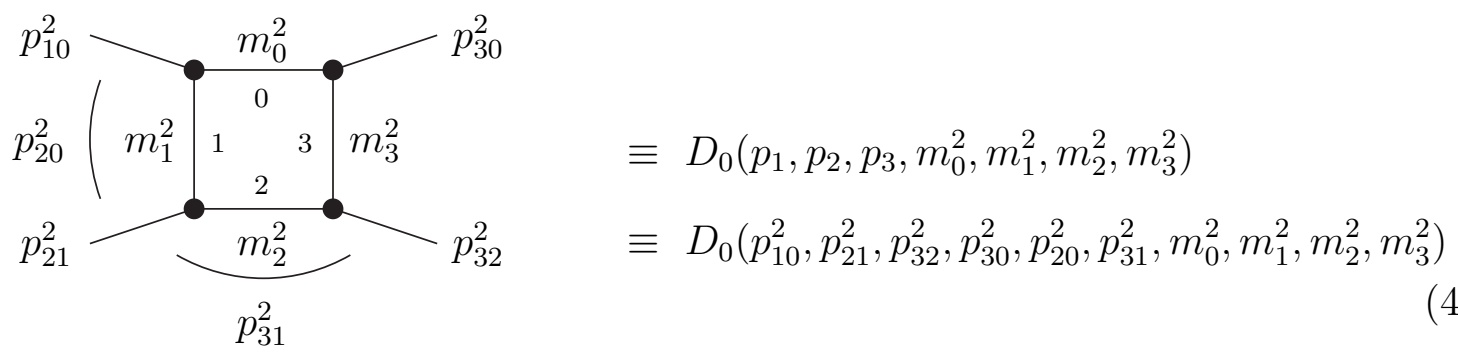

with $p_{i j}=p_{i}-p_{j}$. Over-lined variables are understood to receive an infinitesimally small imaginary part, $\bar{p}_{i j}^{2}=p_{i j}^{2}+\mathrm{i}$, etc., and internal squared masses other than $\lambda^{2}$ or $\lambda_{i}^{2}$ are allowed to be complex with phases between 0 and $-\pi / 2$. We categorise the singular $D_{0}$ functions according to their numbers of collinear and soft singularities, $n_{\text {coll }}$ and $n_{\text {soft }}$, respectively, following the classification of Kinoshita [34]:

- Each of the six squared momenta $p_{i j}^{2}$ gives rise to a collinear singularity if the three variables $p_{i j}^{2}, m_{i}^{2}$, and $m_{j}^{2}$ are zero or small, i.e. of $\mathcal{O}\left(\lambda_{(i)}^{2}\right)$. Pictorially this means that the squared momentum difference between two propagators of small masses is small, e.g. if a particle with a light-like vector couples to two light internal particles. In nonexceptional phase-space points of scattering reactions either $\left\{p_{10}^{2}, p_{32}^{2}\right\}$ or $\left\{p_{21}^{2}, p_{30}^{2}\right\}$ or $\left\{p_{20}^{2}, p_{31}^{2}\right\}$ are not small or zero, so that at most four collinear singularities can be present at a time. By convention we take $\left\{p_{20}^{2}, p_{31}^{2}\right\}$ not small in the following.

- A soft singularity arises if a light particle is exchanged between two on-shell particles. Formally this situation appears if $m_{i}^{2}$ is zero and $p_{i j}^{2}=m_{j}^{2}$ and $p_{i k}^{2}=m_{k}^{2}$ for two different indices $j, k$ not equal to $i$. For non-exceptional phase-space points, at most four soft singularities can exist at a time.

In the following we also define the regularization-scheme-independent finite part $D_{0}^{(\text {fin })}$ of a singular $D_{0}$ function, as described in Ref. [23], upon subtracting an appropriate linear combination of scalar 3-point integrals $C_{0}(i)$, which result from $D_{0}$ upon shrinking propagator $i$ to a point.

(i) Purely soft-singular cases $\left(n_{\text {coll }}=0\right)$

There are two cases with only one soft, but no collinear singularity. Taking $m_{0}$ as the "soft mass", the common finite part of these soft-singular integrals is given by

$$
D_{0}^{(\mathrm{fin})}=D_{0}-\frac{C_{0}(2)}{p_{20}^{2}-m_{2}^{2}} .
$$


Therefore, knowing $D_{0}$ of (4.4) one can obtain $D_{0}$ of (4.5) from the difference of the corresponding integrals $C_{0}(2)$ for $m_{0}=0$ and $m_{0}=\lambda$, which can be found in Appendix B of Ref. [ 23]. The mass-regularized integral (4.5) was taken over from (2.9) [case (i)] of Ref. [33]. The dimensionally regularized integral can be obtained by the simple replacement

$$
\ln (\lambda) \rightarrow \frac{c_{\epsilon}}{2 \epsilon}+\ln (\mu)+\mathcal{O}(\epsilon)
$$

which applies if only soft (but no collinear) singularities are regulated dimensionally, and the results are given by

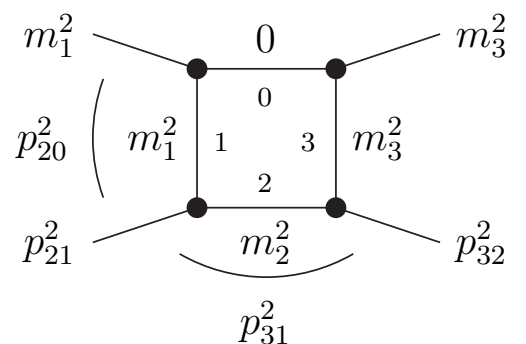

$$
\begin{aligned}
& \left(n_{\mathrm{soft}}=1\right) \\
= & \frac{x_{31}}{m_{1} m_{3}\left(p_{20}^{2}-m_{2}^{2}\right)\left(1-x_{31}^{2}\right)}\left\{2 \operatorname { l n } ( x _ { 3 1 } ) \left[-\frac{c_{\epsilon}}{2 \epsilon}\right.\right. \\
& \left.\quad-\ln \left(\frac{\mu m_{2}}{m_{2}^{2}-\bar{p}_{20}^{2}}\right)+\ln \left(1-x_{31}^{2}\right)\right]+\ln ^{2}\left(x_{21}\right)+\ln ^{2}\left(x_{32}\right)
\end{aligned}
$$$$
\left.+\operatorname{Li}_{2}\left(x_{31}^{2}\right)+\sum_{k, l= \pm 1} \mathcal{L} i_{2}\left(x_{31}, x_{21}^{k}, x_{32}^{l}\right)-\frac{\pi^{2}}{6}\right\} \text {, }
$$

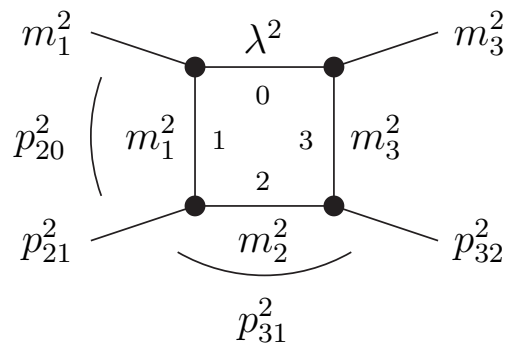

$$
\left(n_{\mathrm{soft}}=1\right)
$$

$$
=\frac{x_{31}}{m_{1} m_{3}\left(p_{20}^{2}-m_{2}^{2}\right)\left(1-x_{31}^{2}\right)}\left\{2 \operatorname { l n } ( x _ { 3 1 } ) \left[-\ln \left(\frac{m_{2} \lambda}{m_{2}^{2}-\bar{p}_{20}^{2}}\right)\right.\right.
$$$$
\left.+\ln \left(1-x_{31}^{2}\right)\right]+\ln ^{2}\left(x_{21}\right)+\ln ^{2}\left(x_{32}\right)+\operatorname{Li}_{2}\left(x_{31}^{2}\right)
$$

$$
\left.+\sum_{k, l= \pm 1} \mathcal{L} i_{2}\left(x_{31}, x_{21}^{k}, x_{32}^{l}\right)-\frac{\pi^{2}}{6}\right\}
$$

where the shorthands $x_{i j}$ of (2.7) are used. Note that the precise definition of $x_{i j}$, which implies $\left|x_{i j}\right| \leq 1$, is important for the analytical continuation. Owing to the on-shell conditions the masses $m_{1}$ and $m_{3}$ are real here, but $m_{2}$ can be complex.

The results for $m_{2}=0$ are obtained by the substitution

$$
\begin{gathered}
-2 \ln \left(x_{31}\right) \ln \left(\frac{m_{2} \nu}{m_{2}^{2}-\bar{p}_{20}^{2}}\right)+\ln ^{2}\left(x_{21}\right)+\ln ^{2}\left(x_{32}\right)+\sum_{k, l= \pm 1} \mathcal{L} i_{2}\left(x_{31}, x_{21}^{k}, x_{32}^{l}\right) \\
\rightarrow \ln \left(x_{31}\right)\left[-\frac{1}{2} \ln \left(x_{31}\right)-\ln \left(\frac{\nu^{2}}{m_{1} m_{3}}\right)-\ln \left(\frac{m_{1}^{2}-\bar{p}_{21}^{2}}{-\bar{p}_{20}^{2}}\right)-\ln \left(\frac{m_{3}^{2}-\bar{p}_{32}^{2}}{-\bar{p}_{20}^{2}}\right)\right] \\
+\frac{1}{2} \ln ^{2}(y)+\sum_{l= \pm 1} \mathcal{L} i_{2}\left(x_{31}, y^{l}\right)
\end{gathered}
$$

with $\nu=\mu$ or $\nu=\lambda$, where

$$
y=\frac{x_{21}}{x_{32}}=\frac{m_{1}\left(m_{3}^{2}-\bar{p}_{32}^{2}\right)}{m_{3}\left(m_{1}^{2}-\bar{p}_{21}^{2}\right)}
$$


The dimensionally regularized integral (4.4) corresponds to "Box 16" of Ref. [ 28 .

The following three cases contain two soft singularities. Their finite part is given by

$$
D_{0}^{(\mathrm{fin})}=D_{0}-\frac{C_{0}(0)+C_{0}(2)}{p_{20}^{2}},
$$

i.e. the full expressions for the $D_{0}$ functions can be deduced from just one out of the three cases upon changing the regularization by subtracting and re-adding 3-point integrals (see Appendix B of Ref. [23]). The purely mass-regularized case (4.11) can be read off from (2.13) [case (iii)] of Ref. [33] (see also Ref. [36]), so that the full results read

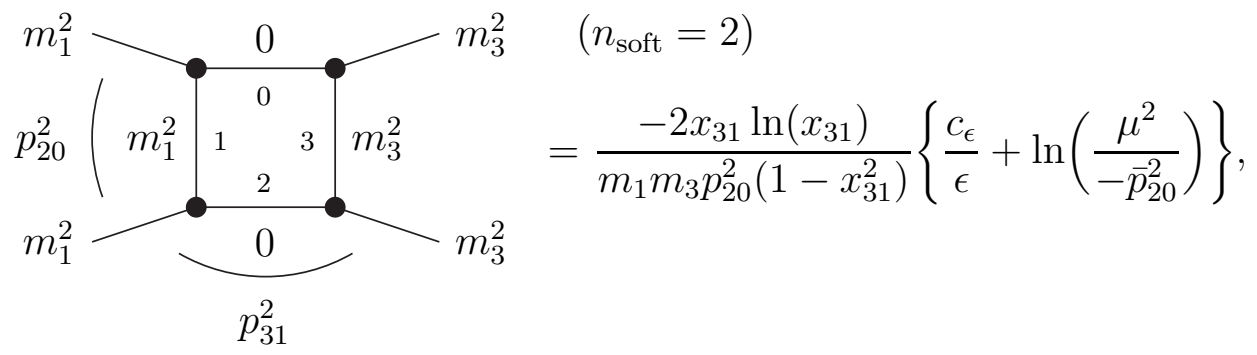

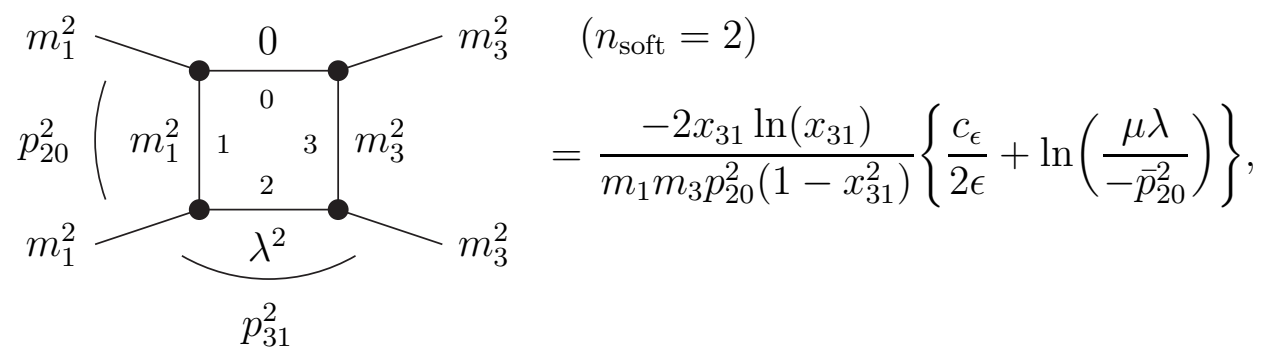

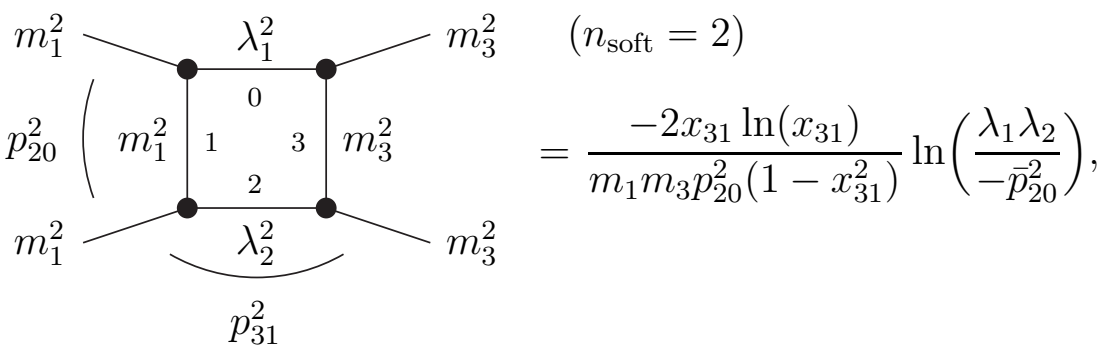

where $x_{31}$ is again defined as in (2.7). Owing to the on-shell conditions the masses $m_{1}$ and $m_{3}$ are real.

The dimensionally regularized integral (4.9) corresponds to "Box 14" of Ref. [28].

(ii) Cases with one collinear singularity $\left(n_{\text {coll }}=1\right)$

The following three cases do not contain soft singularities and have the common finite part

$$
D_{0}^{(\mathrm{fin})}=D_{0}-\frac{\left(p_{31}^{2}-p_{30}^{2}\right) C_{0}(2)+\left(p_{20}^{2}-p_{21}^{2}\right) C_{0}(3)}{\left(p_{20}^{2}-m_{2}^{2}\right)\left(p_{31}^{2}-m_{3}^{2}\right)-\left(p_{21}^{2}-m_{2}^{2}\right)\left(p_{30}^{2}-m_{3}^{2}\right)} .
$$


The following results are valid for complex masses $m_{2}$ and $m_{3}$ and $m_{3} \neq 0$. For (4.13) and (4.15) results for $m_{3}=0$ and $m_{2} \neq 0$ result from a permutation of the arguments, for (4.14) we also give a result that is valid for $m_{3}=0$ and $m_{2} \neq 0$ :

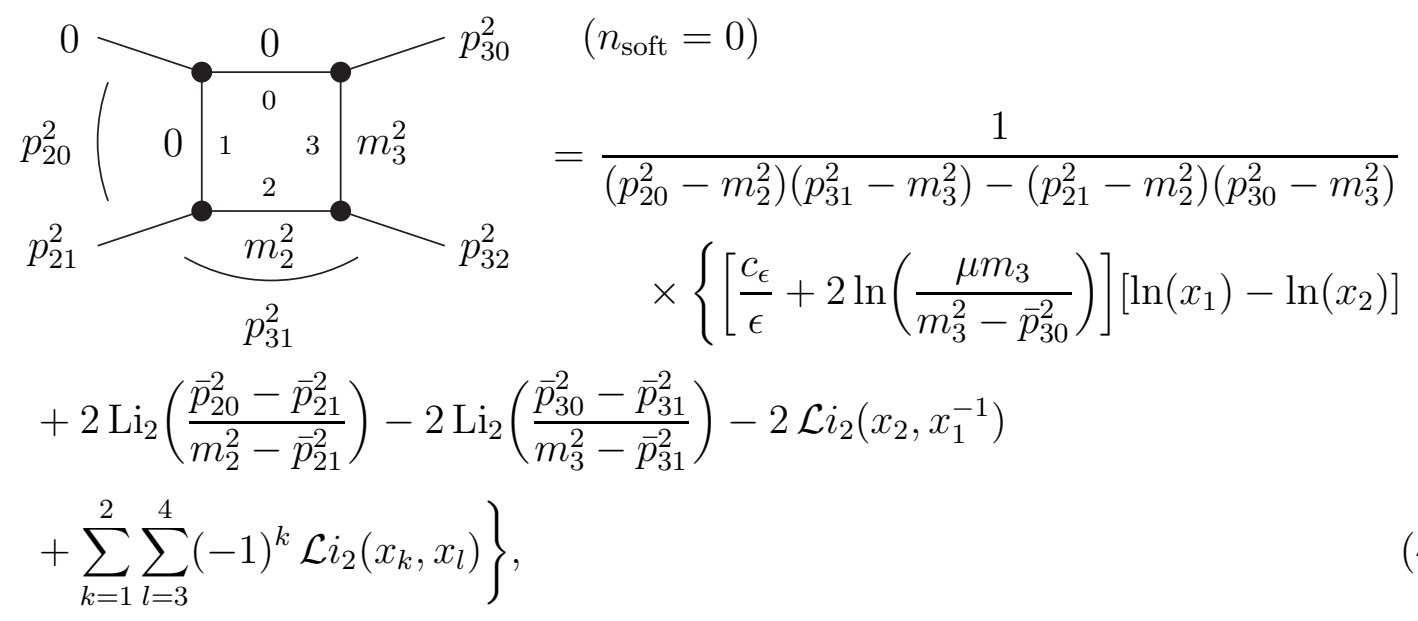

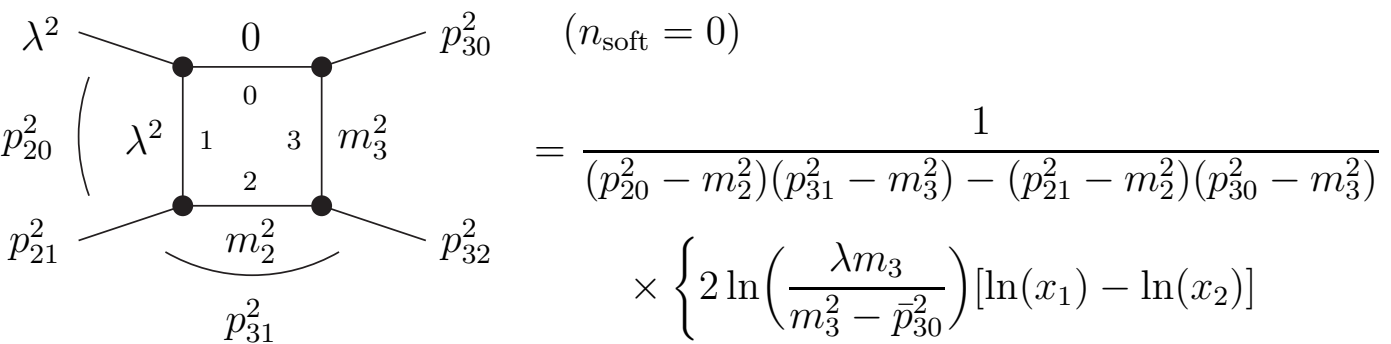

$$
\begin{aligned}
& \left.-\ln ^{2}\left(\frac{m_{2}^{2}-\bar{p}_{20}^{2}}{m_{2}^{2}-\bar{p}_{21}^{2}}\right)+\ln ^{2}\left(\frac{m_{3}^{2}-\bar{p}_{30}^{2}}{m_{3}^{2}-\bar{p}_{31}^{2}}\right)-2 \mathcal{L} i_{2}\left(x_{2}, x_{1}^{-1}\right)+\sum_{k=1}^{2} \sum_{l=3}^{4}(-1)^{k} \mathcal{L} i_{2}\left(x_{k}, x_{l}\right)\right\}
\end{aligned}
$$$$
=\frac{1}{\left(p_{20}^{2}-m_{2}^{2}\right)\left(p_{31}^{2}-m_{3}^{2}\right)-\left(p_{21}^{2}-m_{2}^{2}\right)\left(p_{30}^{2}-m_{3}^{2}\right)}\left\{2 \ln \left(\frac{\lambda m_{2}}{m_{2}^{2}-\bar{p}_{21}^{2}}\right)\left[\ln \left(x_{1}\right)-\ln \left(x_{2}\right)\right]\right.
$$$$
\left.-2 \mathcal{L} i_{2}\left(x_{2}, x_{1}^{-1}\right)-\sum_{k=1}^{2} \sum_{l=3}^{4}(-1)^{k} \mathcal{L} i_{2}\left(x_{k}^{-1}, x_{l}^{-1}\right)\right\},
$$

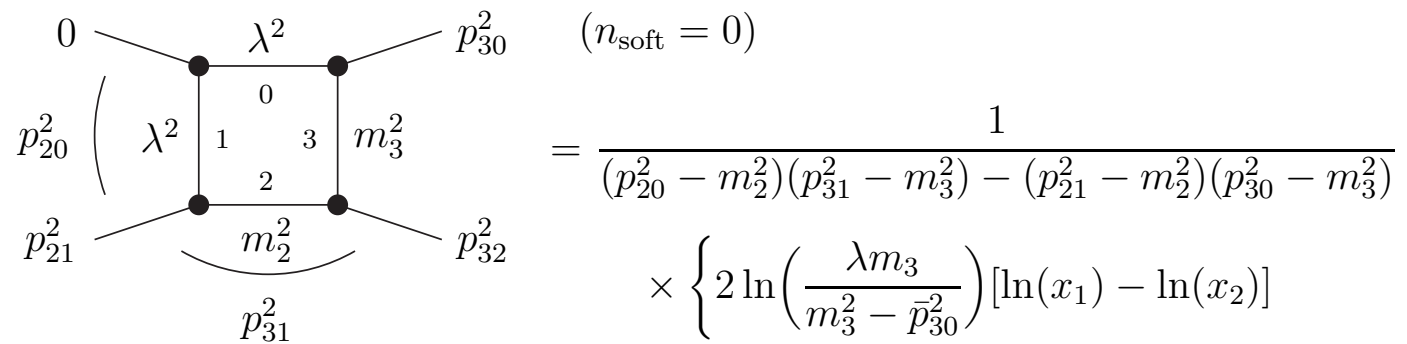

$$
\begin{aligned}
& +2 \operatorname{Li}_{2}\left(\frac{\bar{p}_{20}^{2}-\bar{p}_{21}^{2}}{m_{2}^{2}-\bar{p}_{21}^{2}}\right)-2 \operatorname{Li}_{2}\left(\frac{\bar{p}_{30}^{2}-\bar{p}_{31}^{2}}{m_{3}^{2}-\bar{p}_{31}^{2}}\right)-2 \mathcal{L} i_{2}\left(x_{2}, x_{1}^{-1}\right) \\
& \left.+\sum_{k=1}^{2} \sum_{l=3}^{4}(-1)^{k} \mathcal{L} i_{2}\left(x_{k}, x_{l}\right)\right\} \text {, }
\end{aligned}
$$


with the quantities

$$
\begin{aligned}
x_{1} & =\frac{m_{3}^{2}-\bar{p}_{30}^{2}}{m_{2}^{2}-\bar{p}_{20}^{2}}, \quad x_{2}=\frac{m_{3}^{2}-\bar{p}_{31}^{2}}{m_{2}^{2}-\bar{p}_{21}^{2}}, \\
x_{3,4} & =\frac{m_{2}^{2}+m_{3}^{2}-\bar{p}_{32}^{2} \pm \sqrt{\lambda\left(\bar{p}_{32}^{2}, m_{2}^{2}, m_{3}^{2}\right)}}{2 m_{3}^{2}}=\frac{2 m_{2}^{2}}{m_{2}^{2}+m_{3}^{2}-\bar{p}_{32}^{2} \mp \sqrt{\lambda\left(\bar{p}_{32}^{2}, m_{2}^{2}, m_{3}^{2}\right)}} .
\end{aligned}
$$

We remark that $x_{3}=\left(m_{2} / m_{3}\right) x_{32}^{-1}=1 / r_{23,1}=r_{32,2}, x_{4}=\left(m_{2} / m_{3}\right) x_{32}=1 / r_{23,2}=r_{32,1}$, with $x_{32}$ and $r_{23, k}$ defined in (2.7) and (2.5), respectively.

The results for $m_{2}=m_{3}=0$ are obtained by the substitutions

$$
\begin{aligned}
& \sum_{k=1}^{2} \sum_{l=3}^{4}(-1)^{k} \mathcal{L} i_{2}\left(x_{k}, x_{l}\right)+2 \ln \left(\frac{\nu m_{3}}{m_{3}^{2}-\bar{p}_{30}^{2}}\right)\left[\ln \left(x_{1}\right)-\ln \left(x_{2}\right)\right] \\
& \rightarrow \frac{1}{2} \ln ^{2}\left(x_{1}\right)-\frac{1}{2} \ln ^{2}\left(x_{2}\right)+\left[\ln \left(\frac{\bar{p}_{32}^{2}}{\bar{p}_{30}^{2}}\right)+\ln \left(\frac{\nu^{2}}{-\bar{p}_{30}^{2}}\right)\right]\left[\ln \left(x_{1}\right)-\ln \left(x_{2}\right)\right]
\end{aligned}
$$

with $\nu=\lambda$ or $\nu=\mu$.

The dimensionally regularized integral (4.13) corresponds to "Box 13" of Ref. [28].

The following four cases with no or one soft singularity have the common finite part

$$
D_{0}^{(\mathrm{fin})}=D_{0}-\frac{C_{0}(2)}{p_{20}^{2}-m_{2}^{2}}-\frac{\left(p_{20}^{2}-p_{21}^{2}\right) C_{0}(3)}{\left(p_{20}^{2}-m_{2}^{2}\right)\left(p_{31}^{2}-m_{3}^{2}\right)} .
$$

Here the mass $m_{3}$ is real, but $m_{2}$ can be complex,

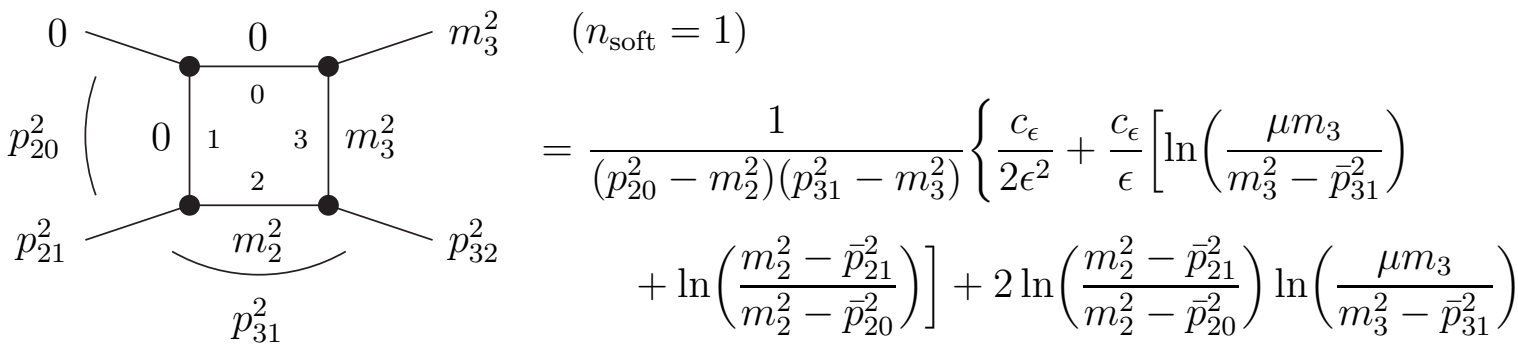

$$
\begin{aligned}
& \left.+\ln ^{2}\left(\frac{\mu m_{3}}{m_{3}^{2}-\bar{p}_{31}^{2}}\right)-2 \operatorname{Li}_{2}\left(\frac{\bar{p}_{21}^{2}-\bar{p}_{20}^{2}}{m_{2}^{2}-\bar{p}_{20}^{2}}\right)+\sum_{l= \pm 1} \mathcal{L} i_{2}\left(\frac{m_{3}^{2}-\bar{p}_{31}^{2}}{m_{2}^{2}-\bar{p}_{21}^{2}}, \frac{m_{2}}{m_{3}} x_{32}^{l}\right)-\frac{\pi^{2}}{6}\right\},
\end{aligned}
$$

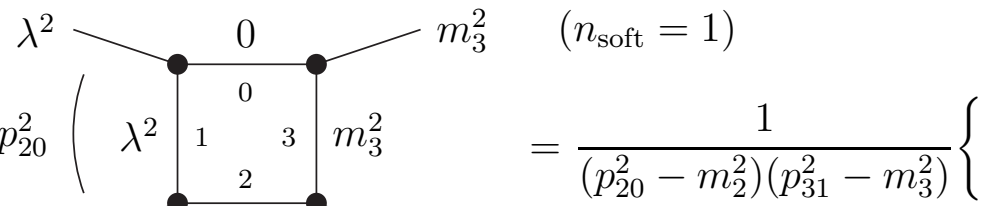

$$
\begin{aligned}
& p_{21}^{2}-\frac{m_{2}^{2}}{p_{31}^{2}} p_{32}^{2} \quad\left[\frac{c_{\epsilon}}{\epsilon}+2 \ln \left(\frac{m_{2}^{2}-\bar{p}_{21}^{2}}{m_{2}^{2}-\bar{p}_{20}^{2}}\right)+\ln \left(\frac{\mu^{2}}{\lambda^{2}}\right)\right] \ln \left(\frac{\lambda m_{3}}{m_{3}^{2}-\bar{p}_{31}^{2}}\right) \\
& \left.+\ln ^{2}\left(\frac{\lambda m_{3}}{m_{3}^{2}-\bar{p}_{31}^{2}}\right)+\sum_{l= \pm 1} \mathcal{L} i_{2}\left(\frac{m_{3}^{2}-\bar{p}_{31}^{2}}{m_{2}^{2}-\bar{p}_{21}^{2}}, \frac{m_{2}}{m_{3}} x_{32}^{l}\right)-\frac{\pi^{2}}{6}\right\},
\end{aligned}
$$




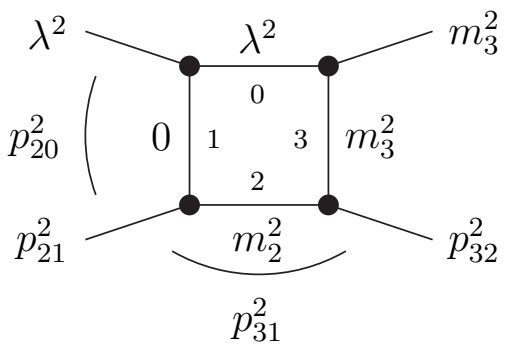

$$
\begin{aligned}
& \left(n_{\text {soft }}=0\right) \\
& =\frac{1}{\left(p_{20}^{2}-m_{2}^{2}\right)\left(p_{31}^{2}-m_{3}^{2}\right)}\{ \\
& {\left[\ln \left(\frac{\lambda m_{3}}{m_{3}^{2}-\bar{p}_{31}^{2}}\right)+\ln \left(\frac{m_{2}^{2}-\bar{p}_{21}^{2}}{m_{2}^{2}-\bar{p}_{20}^{2}}\right)\right]^{2}} \\
& \left.+\sum_{l= \pm 1} \mathcal{L} i_{2}\left(\frac{m_{3}^{2}-\bar{p}_{31}^{2}}{m_{2}^{2}-\bar{p}_{21}^{2}}, \frac{m_{2}}{m_{3}} x_{32}^{l}\right)+\frac{\pi^{2}}{4}\right\},
\end{aligned}
$$

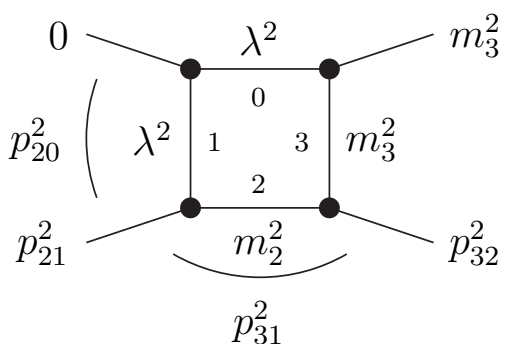

$$
\begin{aligned}
& \left(n_{\mathrm{soft}}=0\right) \\
& =\frac{1}{\left(p_{20}^{2}-m_{2}^{2}\right)\left(p_{31}^{2}-m_{3}^{2}\right)}\{ \\
& {\left[\ln \left(\frac{\lambda m_{3}}{m_{3}^{2}-\bar{p}_{31}^{2}}\right)+\ln \left(\frac{m_{2}^{2}-\bar{p}_{21}^{2}}{m_{2}^{2}-\bar{p}_{20}^{2}}\right)\right]^{2}} \\
& \left.+2 \operatorname{Li}_{2}\left(\frac{\bar{p}_{20}^{2}-\bar{p}_{21}^{2}}{m_{2}^{2}-\bar{p}_{21}^{2}}\right)+\sum_{l= \pm 1} \mathcal{L} i_{2}\left(\frac{m_{3}^{2}-\bar{p}_{31}^{2}}{m_{2}^{2}-\bar{p}_{21}^{2}}, \frac{m_{2}}{m_{3}} x_{32}^{l}\right)-\frac{\pi^{2}}{12}\right\} \text {, }
\end{aligned}
$$

with $x_{32}$ defined as in (2.7). We note that $\left(m_{2} / m_{3}\right) x_{32}^{-1}=1 / r_{23,1}=r_{32,2},\left(m_{2} / m_{3}\right) x_{32}=$ $1 / r_{23,2}=r_{32,1}$, with $r_{i j, k}$ defined in (2.5).

The above results are also valid for $m_{2}=0$, even if $p_{32}^{2}=m_{3}^{2}$. For $m_{2} \rightarrow 0$ the variable $x_{32}$ behaves as

$$
\frac{m_{2}}{m_{3}} x_{32}^{+1}=\mathcal{O}\left(m_{2}^{2}\right), \quad \frac{m_{2}}{m_{3}} x_{32}^{-1}=\frac{m_{3}^{2}-\bar{p}_{32}^{2}}{m_{3}^{2}}+\mathcal{O}\left(m_{2}^{2}\right) .
$$

The dimensionally regularized integral (4.19) can be found in (A.4) of Ref. [35] and corresponds to "Box 12" of Ref. [28]. Integral (4.20) is case (ia) of Ref. [33] (modulo the translation from mass to dimensional regularization which is trivial for the purely soft-singular case) and corresponds to (4.4) for small $m_{1}$.

The following three cases with up to two soft singularities have the common finite part

$$
D_{0}^{(\mathrm{fin})}=D_{0}-\frac{C_{0}(2)}{p_{20}^{2}-m_{2}^{2}}-\frac{C_{0}(3)}{p_{31}^{2}-m_{3}^{2}} .
$$

Here $m_{2}$ and $m_{3}$ have to be real quantities,

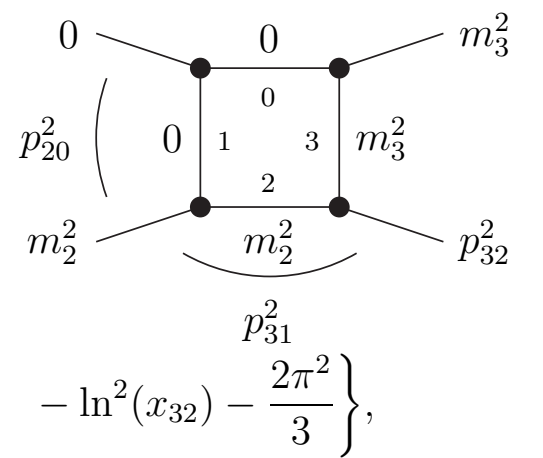

$$
\begin{aligned}
& \left(n_{\mathrm{soft}}=2\right) \\
& =\frac{1}{\left(p_{20}^{2}-m_{2}^{2}\right)\left(p_{31}^{2}-m_{3}^{2}\right)}\left\{\frac{c_{\epsilon}}{\epsilon^{2}}+\frac{c_{\epsilon}}{\epsilon} \ln \left(\frac{\mu m_{2}}{m_{2}^{2}-\bar{p}_{20}^{2}}\right)\right. \\
& \quad+\frac{c_{\epsilon}}{\epsilon} \ln \left(\frac{\mu m_{3}}{m_{3}^{2}-\bar{p}_{31}^{2}}\right)+2 \ln \left(\frac{\mu m_{2}}{m_{2}^{2}-\bar{p}_{20}^{2}}\right) \ln \left(\frac{\mu m_{3}}{m_{3}^{2}-\bar{p}_{31}^{2}}\right)
\end{aligned}
$$




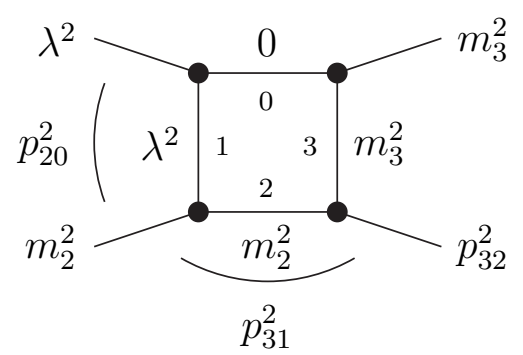

$$
\begin{aligned}
& \left(n_{\mathrm{soft}}=1\right) \\
& =\frac{1}{\left(p_{20}^{2}-m_{2}^{2}\right)\left(p_{31}^{2}-m_{3}^{2}\right)}\left\{\frac{c_{\epsilon}}{\epsilon} \ln \left(\frac{\lambda m_{3}}{m_{3}^{2}-\bar{p}_{31}^{2}}\right)\right. \\
& \left.\quad+2 \ln \left(\frac{\mu m_{2}}{m_{2}^{2}-\bar{p}_{20}^{2}}\right) \ln \left(\frac{\lambda m_{3}}{m_{3}^{2}-\bar{p}_{31}^{2}}\right)-\ln ^{2}\left(x_{32}\right)-\frac{\pi^{2}}{4}\right\},
\end{aligned}
$$

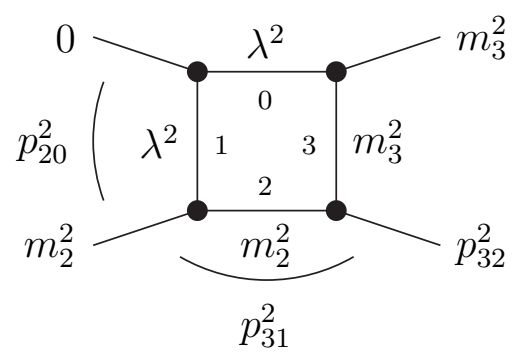

$$
\begin{aligned}
& \left(n_{\mathrm{soft}}=0\right) \\
& =\frac{1}{\left(p_{20}^{2}-m_{2}^{2}\right)\left(p_{31}^{2}-m_{3}^{2}\right)}\left\{2 \ln \left(\frac{\lambda m_{2}}{m_{2}^{2}-\bar{p}_{20}^{2}}\right) \ln \left(\frac{\lambda m_{3}}{m_{3}^{2}-\bar{p}_{31}^{2}}\right)\right. \\
& \left.\quad-\ln ^{2}\left(x_{32}\right)-\frac{\pi^{2}}{2}\right\}
\end{aligned}
$$

with again $x_{32}$ defined as in (2.7).

The dimensionally regularized integral (4.25) can be found in (A.4) of Ref. [35] and corresponds to "Box 11" of Ref. [28]. Integral (4.26) can be calculated from case (i) of Ref. [33] for $m_{0}^{2}=m_{2}^{2}$ and $m_{1}$ small (with the trivial translation to dimensional regularization) or from (4.4) for $p_{21}^{2}=m_{2}^{2}$ and small $m_{1}$.

(iii) Cases with two collinear singularities on adjacent legs $\left(n_{\text {coll }}=2\right)$

Here we distinguish six different cases with no or one soft singularity which have the following common finite part

$$
D_{0}^{(\mathrm{fin})}=D_{0}-\frac{\left(p_{31}^{2}-p_{32}^{2}\right) C_{0}(0)+\left(p_{31}^{2}-p_{30}^{2}\right) C_{0}(2)}{p_{20}^{2}\left(p_{31}^{2}-m_{3}^{2}\right)}-\frac{C_{0}(3)}{p_{31}^{2}-m_{3}^{2}} .
$$

The mass $m_{3}$ can be complex,

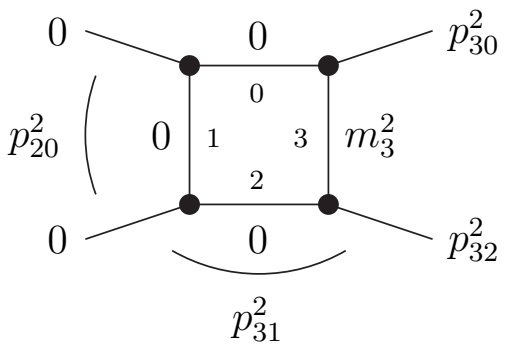

$$
\begin{aligned}
& \left(n_{\mathrm{soft}}=1\right) \\
& =\frac{1}{p_{20}^{2}\left(p_{31}^{2}-m_{3}^{2}\right)}\left\{\frac{c_{\epsilon}}{\epsilon^{2}}+\frac{c_{\epsilon}}{\epsilon}\left[\ln \left(\frac{\mu^{2}}{-\bar{p}_{20}^{2}}\right)+\ln \left(\frac{m_{3}^{2}-\bar{p}_{32}^{2}}{m_{3}^{2}-\bar{p}_{31}^{2}}\right)\right.\right. \\
& \left.+\ln \left(\frac{m_{3}^{2}-\bar{p}_{30}^{2}}{m_{3}^{2}-\bar{p}_{31}^{2}}\right)\right]+\frac{1}{2} \ln ^{2}\left(\frac{\mu^{2}}{-\bar{p}_{20}^{2}}\right) \\
& +\ln \left(\frac{\mu^{2}}{-\bar{p}_{20}^{2}}\right)\left[\ln \left(\frac{m_{3}^{2}-\bar{p}_{32}^{2}}{m_{3}^{2}-\bar{p}_{31}^{2}}\right)+\ln \left(\frac{m_{3}^{2}-\bar{p}_{30}^{2}}{m_{3}^{2}-\bar{p}_{31}^{2}}\right)\right]-\frac{1}{2} \ln ^{2}\left(\frac{m_{3}^{2}-\bar{p}_{32}^{2}}{m_{3}^{2}-\bar{p}_{30}^{2}}\right) \\
& \left.-2 \operatorname{Li}_{2}\left(\frac{\bar{p}_{32}^{2}-\bar{p}_{31}^{2}}{m_{3}^{2}-\bar{p}_{31}^{2}}\right)-2 \operatorname{Li}_{2}\left(\frac{\bar{p}_{30}^{2}-\bar{p}_{31}^{2}}{m_{3}^{2}-\bar{p}_{31}^{2}}\right)+\mathcal{L} i_{2}\left(\frac{\bar{p}_{20}^{2}}{\bar{p}_{32}^{2}-m_{3}^{2}}, \frac{m_{3}^{2}}{m_{3}^{2}-\bar{p}_{30}^{2}}\right)-\frac{\pi^{2}}{3}\right\},
\end{aligned}
$$




$$
\begin{aligned}
& \lambda^{2} \longrightarrow \lambda^{2} \smile p_{30}^{2} \quad\left(n_{\text {soft }}=1\right)
\end{aligned}
$$

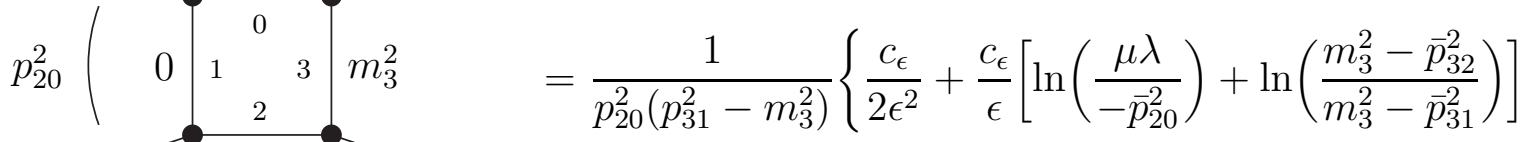

$$
\begin{aligned}
& -\ln ^{2}\left(\frac{\mu}{\lambda}\right)+\frac{1}{2} \ln ^{2}\left(\frac{\mu^{2}}{-\bar{p}_{20}^{2}}\right) \\
& +\ln \left(\frac{\mu^{2}}{-\bar{p}_{20}^{2}}\right) \ln \left(\frac{m_{3}^{2}-\bar{p}_{32}^{2}}{m_{3}^{2}-\bar{p}_{31}^{2}}\right)+\ln \left(\frac{\lambda^{2}}{-\bar{p}_{20}^{2}}\right) \ln \left(\frac{m_{3}^{2}-\bar{p}_{30}^{2}}{m_{3}^{2}-\bar{p}_{31}^{2}}\right)-\frac{1}{2} \ln ^{2}\left(\frac{m_{3}^{2}-\bar{p}_{32}^{2}}{m_{3}^{2}-\bar{p}_{30}^{2}}\right) \\
& \left.-2 \operatorname{Li}_{2}\left(\frac{\bar{p}_{32}^{2}-\bar{p}_{31}^{2}}{m_{3}^{2}-\bar{p}_{31}^{2}}\right)+\mathcal{L} i_{2}\left(\frac{\bar{p}_{20}^{2}}{\bar{p}_{32}^{2}-m_{3}^{2}}, \frac{m_{3}^{2}}{m_{3}^{2}-\bar{p}_{30}^{2}}\right)-\frac{\pi^{2}}{3}\right\} \\
& \lambda_{1}^{2} \longrightarrow \lambda_{1}^{2} \smile p_{30}^{2} \quad\left(n_{\text {soft }}=1\right)
\end{aligned}
$$

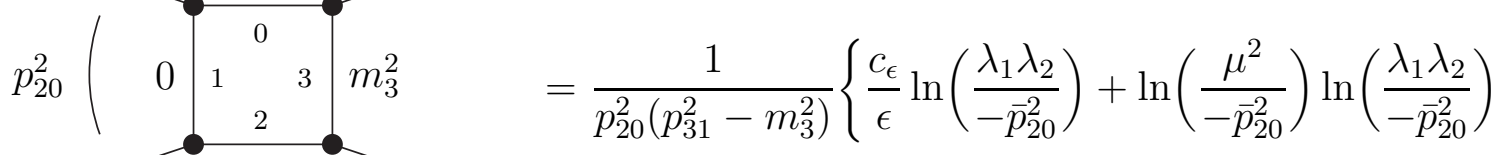

$$
\begin{aligned}
& \lambda_{2}^{2}-p_{32}^{2}-\frac{1}{p_{31}^{2}} \ln ^{2}\left(\frac{\lambda_{1}^{2}}{-\bar{p}_{20}^{2}}\right)-\frac{1}{4} \ln ^{2}\left(\frac{\lambda_{2}^{2}}{-\bar{p}_{20}^{2}}\right) \\
& +\ln \left(\frac{\lambda_{1}^{2}}{-\bar{p}_{20}^{2}}\right) \ln \left(\frac{m_{3}^{2}-\bar{p}_{30}^{2}}{m_{3}^{2}-\bar{p}_{31}^{2}}\right)+\ln \left(\frac{\lambda_{2}^{2}}{-\bar{p}_{20}^{2}}\right) \ln \left(\frac{m_{3}^{2}-\bar{p}_{32}^{2}}{m_{3}^{2}-\bar{p}_{31}^{2}}\right)-\frac{1}{2} \ln ^{2}\left(\frac{m_{3}^{2}-\bar{p}_{32}^{2}}{m_{3}^{2}-\bar{p}_{30}^{2}}\right) \\
& \left.+\mathcal{L} i_{2}\left(\frac{\bar{p}_{20}^{2}}{\bar{p}_{32}^{2}-m_{3}^{2}}, \frac{m_{3}^{2}}{m_{3}^{2}-\bar{p}_{30}^{2}}\right)-\frac{\pi^{2}}{3}\right\} \\
& \lambda^{2} \longrightarrow \overbrace{0} \multimap p_{30}^{2} \quad\left(n_{\text {soft }}=0\right)
\end{aligned}
$$

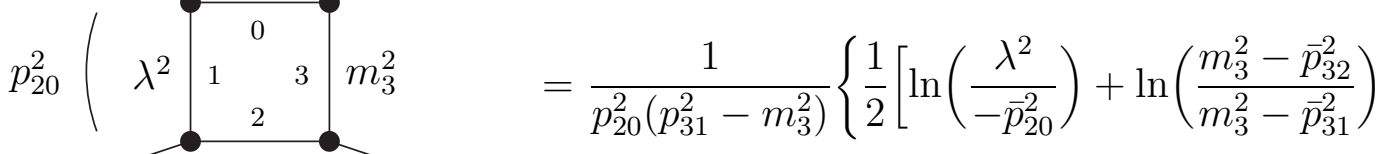

$$
\begin{aligned}
& \left.\lambda^{2}-p_{32}^{2}+\ln \left(\frac{m_{3}^{2}-\bar{p}_{30}^{2}}{m_{3}^{2}-\bar{p}_{31}^{2}}\right)\right]^{2} \\
& \left.+\mathcal{L} i_{2}\left(\frac{\bar{p}_{20}^{2}}{\bar{p}_{32}^{2}-m_{3}^{2}}, \frac{m_{3}^{2}}{m_{3}^{2}-\bar{p}_{30}^{2}}\right)+\frac{\pi^{2}}{2}\right\}
\end{aligned}
$$

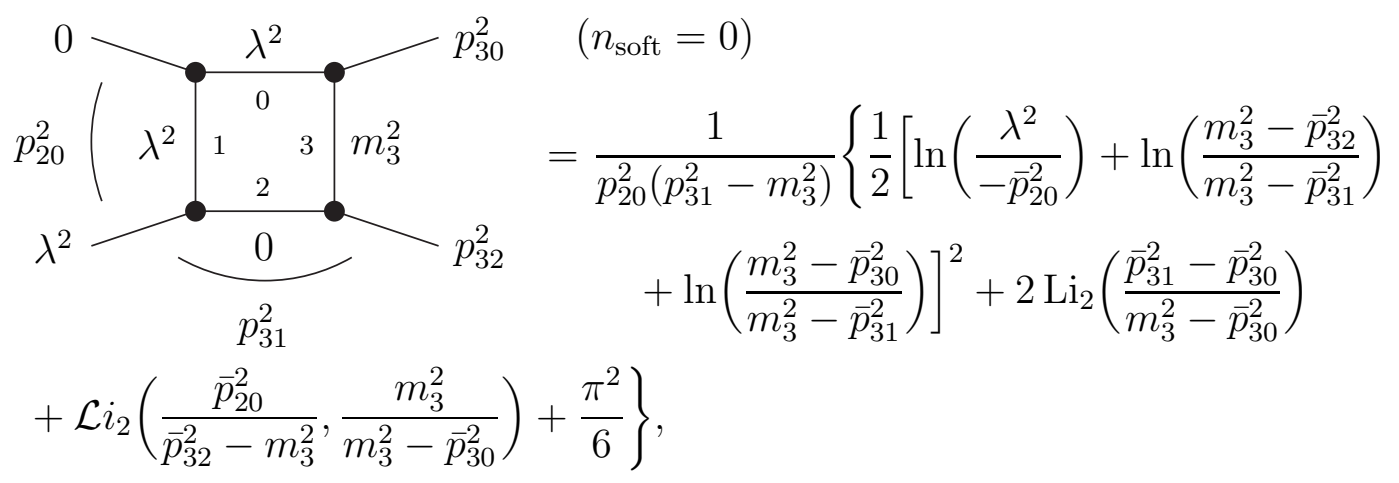




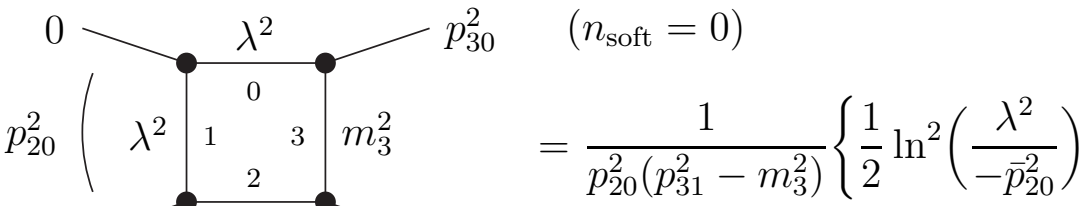

$$
\begin{aligned}
& +\ln \left(\frac{\lambda^{2}}{-\bar{p}_{20}^{2}}\right)\left[\ln \left(\frac{m_{3}^{2}-\bar{p}_{32}^{2}}{m_{3}^{2}-\bar{p}_{31}^{2}}\right)+\ln \left(\frac{m_{3}^{2}-\bar{p}_{30}^{2}}{m_{3}^{2}-\bar{p}_{31}^{2}}\right)\right] \\
& -\frac{1}{2} \ln ^{2}\left(\frac{m_{3}^{2}-\bar{p}_{32}^{2}}{m_{3}^{2}-\bar{p}_{30}^{2}}\right)-2 \operatorname{Li}_{2}\left(\frac{\bar{p}_{32}^{2}-\bar{p}_{31}^{2}}{m_{3}^{2}-\bar{p}_{31}^{2}}\right)-2 \operatorname{Li}_{2}\left(\frac{\bar{p}_{30}^{2}-\bar{p}_{31}^{2}}{m_{3}^{2}-\bar{p}_{31}^{2}}\right) \\
& \left.+\mathcal{L} i_{2}\left(\frac{\bar{p}_{20}^{2}}{\bar{p}_{32}^{2}-m_{3}^{2}}, \frac{m_{3}^{2}}{m_{3}^{2}-\bar{p}_{30}^{2}}\right)-\frac{\pi^{2}}{6}\right\} \text {. }
\end{aligned}
$$

The dimensionally regularized integral (4.29) was calculated in Ref. [ 37] and corresponds to "Box 8" of Ref. [ 28. Integral (4.30) can be obtained from (4.19) for $m_{3}$ small. Integral (4.31) is case (ib) of Ref. [33] (modulo the trivial translation to dimensional regularization) and corresponds to (4.20) for small $m_{3}$.

The following eight different cases with up to two soft singularities have the common finite part

$$
D_{0}^{(\mathrm{fin})}=D_{0}-\frac{\left(p_{31}^{2}-p_{32}^{2}\right) C_{0}(0)}{p_{20}^{2}\left(p_{31}^{2}-m_{3}^{2}\right)}-\frac{C_{0}(2)}{p_{20}^{2}}-\frac{C_{0}(3)}{p_{31}^{2}-m_{3}^{2}},
$$

with the real mass $m_{3}$,

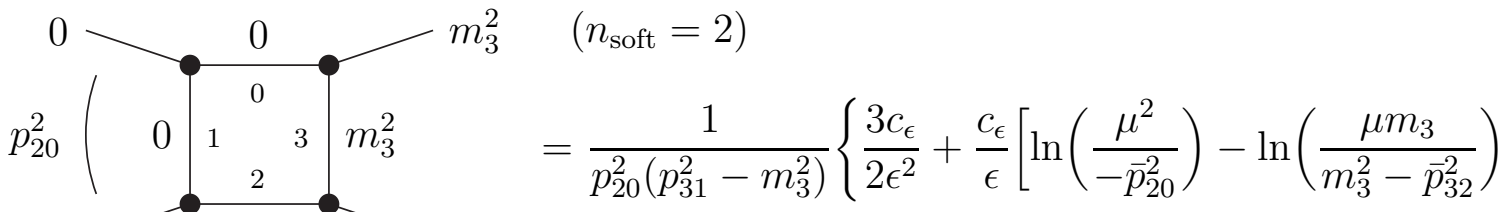

$$
\begin{aligned}
& \left.+2 \ln \left(\frac{\mu m_{3}}{m_{3}^{2}-\bar{p}_{31}^{2}}\right)\right]-\ln ^{2}\left(\frac{\mu m_{3}}{m_{3}^{2}-\bar{p}_{32}^{2}}\right) \\
& \left.+2 \ln \left(\frac{\mu^{2}}{-\bar{p}_{20}^{2}}\right) \ln \left(\frac{\mu m_{3}}{m_{3}^{2}-\bar{p}_{31}^{2}}\right)-2 \operatorname{Li}_{2}\left(\frac{\bar{p}_{32}^{2}-\bar{p}_{31}^{2}}{m_{3}^{2}-\bar{p}_{31}^{2}}\right)-\frac{2 \pi^{2}}{3}\right\},
\end{aligned}
$$

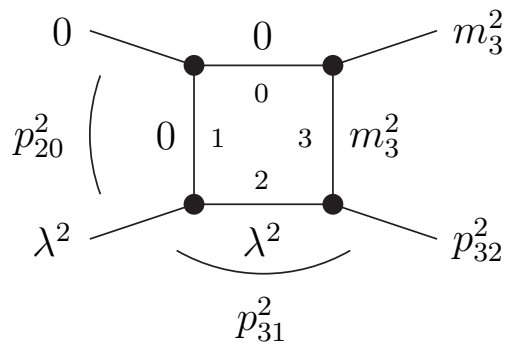

$$
\begin{aligned}
& \left(n_{\mathrm{soft}}=2\right) \\
& =\frac{1}{p_{20}^{2}\left(p_{31}^{2}-m_{3}^{2}\right)}\left\{\frac{c_{\epsilon}}{\epsilon^{2}}+\frac{c_{\epsilon}}{\epsilon}\left[\ln \left(\frac{\mu \lambda}{-\bar{p}_{20}^{2}}\right)+\ln \left(\frac{\mu m_{3}}{m_{3}^{2}-\bar{p}_{31}^{2}}\right)\right]\right. \\
& +2 \ln \left(\frac{\mu \lambda}{-\bar{p}_{20}^{2}}\right) \ln \left(\frac{\mu m_{3}}{m_{3}^{2}-\bar{p}_{31}^{2}}\right)-\ln ^{2}\left(\frac{\lambda m_{3}}{m_{3}^{2}-\bar{p}_{32}^{2}}\right) \\
& \left.-\frac{2 \pi^{2}}{3}\right\}
\end{aligned}
$$




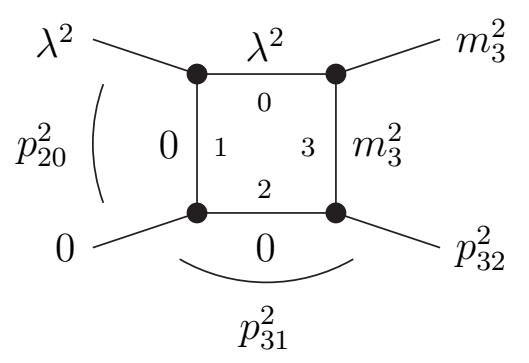

$$
\begin{aligned}
& \left(n_{\text {soft }}=1\right) \\
& =\frac{1}{p_{20}^{2}\left(p_{31}^{2}-m_{3}^{2}\right)}\left\{\frac{c_{\epsilon}}{2 \epsilon^{2}}+\frac{c_{\epsilon}}{\epsilon}\left[\ln \left(\frac{\mu \lambda}{-\bar{p}_{20}^{2}}\right)+\ln \left(\frac{m_{3}^{2}-\bar{p}_{32}^{2}}{m_{3}^{2}-\bar{p}_{31}^{2}}\right)\right]\right. \\
& \quad-\ln ^{2}\left(\frac{\mu m_{3}}{m_{3}^{2}-\bar{p}_{32}^{2}}\right)+2 \ln \left(\frac{\mu \lambda}{-\bar{p}_{20}^{2}}\right) \ln \left(\frac{\mu m_{3}}{m_{3}^{2}-\bar{p}_{31}^{2}}\right) \\
& \left.\quad-2 \operatorname{Li}_{2}\left(\frac{\bar{p}_{32}^{2}-\bar{p}_{31}^{2}}{m_{3}^{2}-\bar{p}_{31}^{2}}\right)-\frac{\pi^{2}}{4}\right\}
\end{aligned}
$$

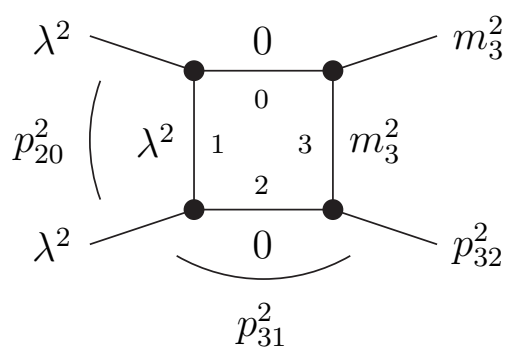

$$
\begin{aligned}
& \left(n_{\mathrm{soft}}=1\right) \\
& =\frac{1}{p_{20}^{2}\left(p_{31}^{2}-m_{3}^{2}\right)}\left\{\left[\frac{c_{\epsilon}}{\epsilon}+2 \ln \left(\frac{\bar{p}_{32}^{2}-m_{3}^{2}}{\bar{p}_{20}^{2}}\right)+\ln \left(\frac{\mu^{2}}{m_{3}^{2}}\right)\right]\right. \\
& \left.\quad \times \ln \left(\frac{\lambda m_{3}}{m_{3}^{2}-\bar{p}_{31}^{2}}\right)+\ln ^{2}\left(\frac{\lambda m_{3}}{m_{3}^{2}-\bar{p}_{31}^{2}}\right)+\frac{\pi^{2}}{6}\right\}
\end{aligned}
$$

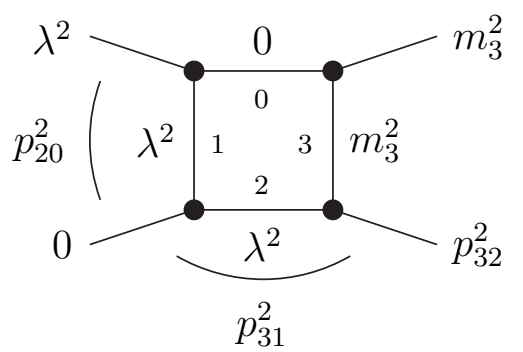

$$
\begin{aligned}
& \left(n_{\mathrm{soft}}=1\right) \\
& =\frac{1}{p_{20}^{2}\left(p_{31}^{2}-m_{3}^{2}\right)}\left\{\left[\frac{c_{\epsilon}}{\epsilon}+2 \ln \left(\frac{\mu \lambda}{-\bar{p}_{20}^{2}}\right)\right] \ln \left(\frac{\lambda m_{3}}{m_{3}^{2}-\bar{p}_{31}^{2}}\right)\right. \\
& \left.\quad-\ln ^{2}\left(\frac{\lambda m_{3}}{m_{3}^{2}-\bar{p}_{32}^{2}}\right)-2 \operatorname{Li}_{2}\left(\frac{\bar{p}_{32}^{2}-\bar{p}_{31}^{2}}{m_{3}^{2}-\bar{p}_{31}^{2}}\right)-\frac{\pi^{2}}{6}\right\}
\end{aligned}
$$

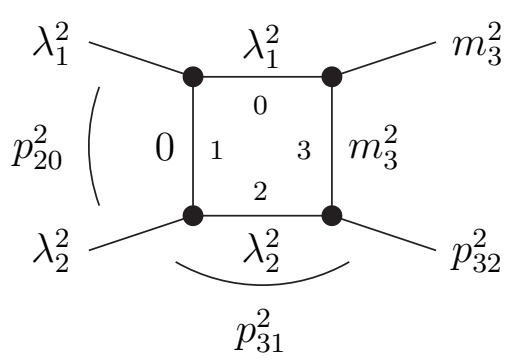

$$
\begin{aligned}
& \left(n_{\mathrm{soft}}=1\right) \\
& =\frac{1}{p_{20}^{2}\left(p_{31}^{2}-m_{3}^{2}\right)}\left\{\left[\frac{c_{\epsilon}}{\epsilon}+2 \ln \left(\frac{\mu m_{3}}{m_{3}^{2}-\bar{p}_{31}^{2}}\right)\right] \ln \left(\frac{\lambda_{1} \lambda_{2}}{-\bar{p}_{20}^{2}}\right)\right. \\
& \left.\quad-\ln ^{2}\left(\frac{\lambda_{2} m_{3}}{m_{3}^{2}-\bar{p}_{32}^{2}}\right)-\frac{\pi^{2}}{4}\right\}
\end{aligned}
$$

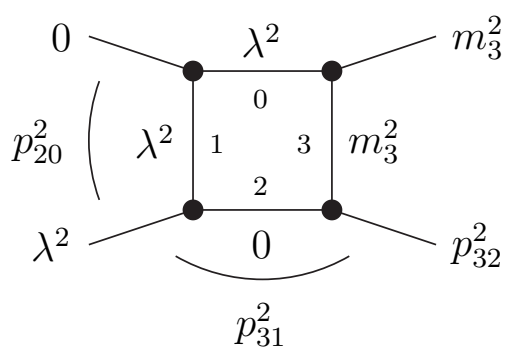

$$
\begin{aligned}
& \left(n_{\mathrm{soft}}=0\right) \\
& =\frac{1}{p_{20}^{2}\left(p_{31}^{2}-m_{3}^{2}\right)}\left\{\operatorname { l n } ( \frac { \lambda m _ { 3 } } { m _ { 3 } ^ { 2 } - \overline { p } _ { 3 1 } ^ { 2 } } ) \left[\ln \left(\frac{\lambda m_{3}}{m_{3}^{2}-\bar{p}_{31}^{2}}\right)\right.\right. \\
& \left.\left.\quad+2 \ln \left(\frac{\lambda^{2}}{-\bar{p}_{20}^{2}}\right)-2 \ln \left(\frac{\lambda m_{3}}{m_{3}^{2}-\bar{p}_{32}^{2}}\right)\right]-\frac{\pi^{2}}{12}\right\},
\end{aligned}
$$




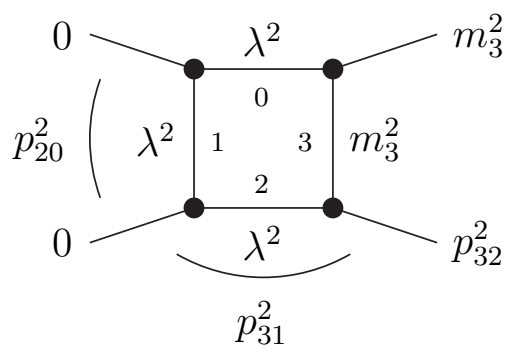

$$
\begin{aligned}
& \left(n_{\mathrm{soft}}=0\right) \\
& =\frac{1}{p_{20}^{2}\left(p_{31}^{2}-m_{3}^{2}\right)}\left\{2 \ln \left(\frac{\lambda^{2}}{-\bar{p}_{20}^{2}}\right) \ln \left(\frac{\lambda m_{3}}{m_{3}^{2}-\bar{p}_{31}^{2}}\right)\right. \\
& \left.\quad-\ln ^{2}\left(\frac{\lambda m_{3}}{m_{3}^{2}-\bar{p}_{32}^{2}}\right)-2 \operatorname{Li}_{2}\left(\frac{\bar{p}_{32}^{2}-\bar{p}_{31}^{2}}{m_{3}^{2}-\bar{p}_{31}^{2}}\right)-\frac{5 \pi^{2}}{12}\right\} .
\end{aligned}
$$

The dimensionally regularized integral (4.36), e.g., appeared in the NLO QCD correction to $\mathrm{pp} \rightarrow \mathrm{t} \overline{\mathrm{t}} \mathrm{H}$ [ 35] [see Eq. (A.4) there] and corresponds to "Box 7" of Ref. [28]. Integral (4.37) can be obtained from (4.25) for $m_{2}$ small. Integral (4.38) can be obtained from (4.19) for $p_{32}^{2}=m_{2}^{2}$ and $m_{3}$ small. Integral (4.39) is case (iic) of Ref. [33] (modulo translation to dimensional regularization) and corresponds to (4.4) with $p_{21}^{2}=m_{1}^{2}$ small and $m_{2}=0$. Similar comments apply to the next two cases. Integral (4.40) results from case (ic) of Ref. [33] and corresponds to (4.4) with $p_{21}^{2}=0$ and $m_{2}=m_{1}$ both small. Integral (4.41) coincides with case (i) of Ref. [33] for $m_{0}^{2}=m_{2}^{2}$ and both $m_{1}$ and $m_{4}$ small and corresponds to (4.4) with $p_{32}^{2}=m_{2}^{2}$ and both $m_{1}$ and $m_{3}$ small.

The following six different cases with up to three soft singularities have the common finite part

$$
D_{0}^{(\mathrm{fin})}=D_{0}-\frac{C_{0}(0)+C_{0}(2)}{p_{20}^{2}}-\frac{C_{0}(3)}{p_{31}^{2}-m_{3}^{2}} .
$$

Again the mass $m_{3}$ has to be a real quantity here,

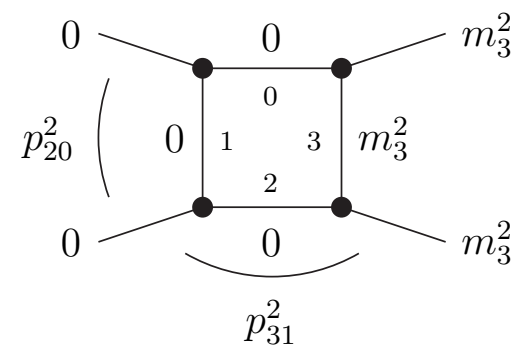

$$
\begin{aligned}
& \left(n_{\mathrm{soft}}=3\right) \\
& =\frac{1}{p_{20}^{2}\left(p_{31}^{2}-m_{3}^{2}\right)}\left\{\frac{2 c_{\epsilon}}{\epsilon^{2}}+\frac{c_{\epsilon}}{\epsilon}\left[\ln \left(\frac{\mu^{2}}{-\bar{p}_{20}^{2}}\right)+2 \ln \left(\frac{\mu m_{3}}{m_{3}^{2}-\bar{p}_{31}^{2}}\right)\right]\right. \\
& \left.\quad+2 \ln \left(\frac{\mu^{2}}{-\bar{p}_{20}^{2}}\right) \ln \left(\frac{\mu m_{3}}{m_{3}^{2}-\bar{p}_{31}^{2}}\right)-\frac{5 \pi^{2}}{6}\right\},
\end{aligned}
$$

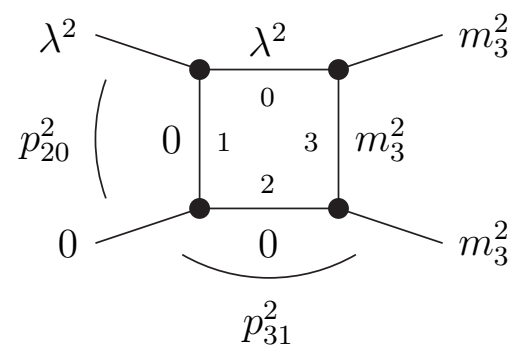

$$
\begin{aligned}
& \left(n_{\text {soft }}=2\right) \\
& =\frac{1}{p_{20}^{2}\left(p_{31}^{2}-m_{3}^{2}\right)}\left\{\frac{c_{\epsilon}}{\epsilon^{2}}+\frac{c_{\epsilon}}{\epsilon}\left[\ln \left(\frac{\mu \lambda}{-\bar{p}_{20}^{2}}\right)+\ln \left(\frac{\mu m_{3}}{m_{3}^{2}-\bar{p}_{31}^{2}}\right)\right]\right. \\
& \left.\quad+2 \ln \left(\frac{\mu \lambda}{-\bar{p}_{20}^{2}}\right) \ln \left(\frac{\mu m_{3}}{m_{3}^{2}-\bar{p}_{31}^{2}}\right)-\frac{5 \pi^{2}}{12}\right\}
\end{aligned}
$$

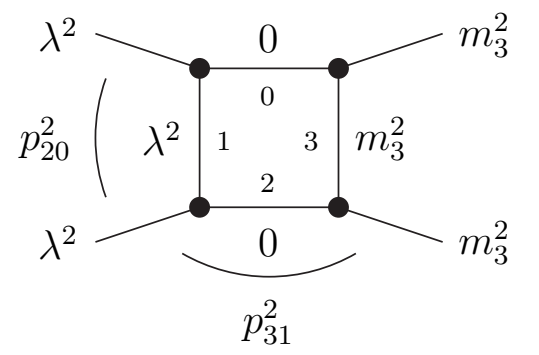

$$
\begin{aligned}
& \left(n_{\mathrm{soft}}=2\right) \\
= & \frac{2}{p_{20}^{2}\left(p_{31}^{2}-m_{3}^{2}\right)}\left[\frac{c_{\epsilon}}{\epsilon}+\ln \left(\frac{\mu^{2}}{-\bar{p}_{20}^{2}}\right)\right] \ln \left(\frac{\lambda m_{3}}{m_{3}^{2}-\bar{p}_{31}^{2}}\right),
\end{aligned}
$$




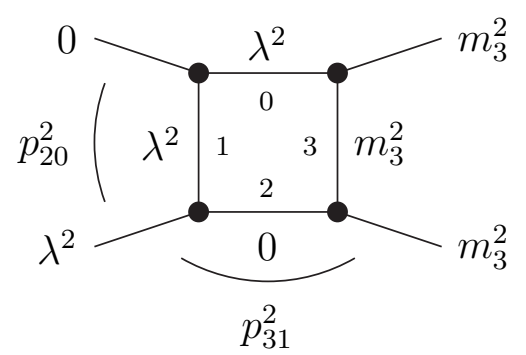

$$
\begin{aligned}
& \left(n_{\mathrm{soft}}=1\right) \\
& =\frac{1}{p_{20}^{2}\left(p_{31}^{2}-m_{3}^{2}\right)}\left\{\left[\frac{c_{\epsilon}}{\epsilon}+2 \ln \left(\frac{\mu \lambda}{-\bar{p}_{20}^{2}}\right)\right] \ln \left(\frac{\lambda m_{3}}{m_{3}^{2}-\bar{p}_{31}^{2}}\right)\right. \\
& \left.\quad-\frac{\pi^{2}}{4}\right\}
\end{aligned}
$$

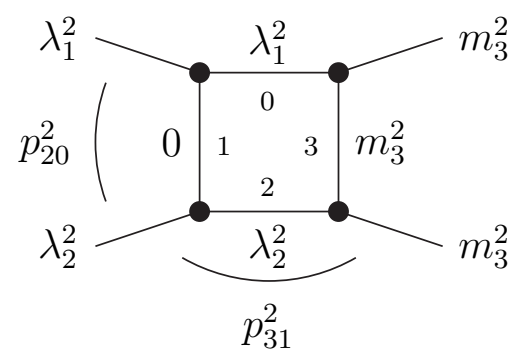

$$
\begin{aligned}
& \left(n_{\mathrm{soft}}=1\right) \\
= & \frac{1}{p_{20}^{2}\left(p_{31}^{2}-m_{3}^{2}\right)}\left[\frac{c_{\epsilon}}{\epsilon}+2 \ln \left(\frac{\mu m_{3}}{m_{3}^{2}-\bar{p}_{31}^{2}}\right)\right] \ln \left(\frac{\lambda_{1} \lambda_{2}}{-\bar{p}_{20}^{2}}\right),
\end{aligned}
$$

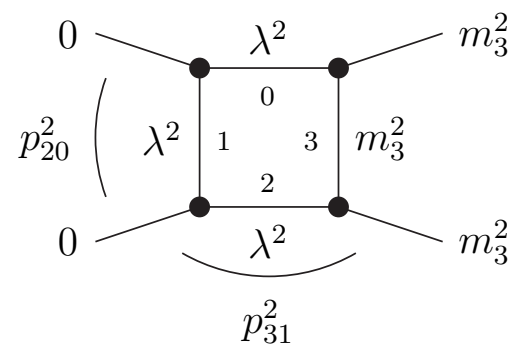

$$
\begin{aligned}
& \left(n_{\mathrm{soft}}=0\right) \\
= & \frac{1}{p_{20}^{2}\left(p_{31}^{2}-m_{3}^{2}\right)}\left\{2 \ln \left(\frac{\lambda^{2}}{-\bar{p}_{20}^{2}}\right) \ln \left(\frac{\lambda m_{3}}{m_{3}^{2}-\bar{p}_{31}^{2}}\right)-\frac{\pi^{2}}{2}\right\} .
\end{aligned}
$$

The integral (4.45) was given in Eq. (A.4) of Ref. [38] and corresponds to "Box 6" of Ref. [28]. Integral (4.46) can be obtained from (4.25) for $p_{32}^{2}=m_{2}^{2}$ and $m_{3}$ small. Integral (4.47) is case (iiia) of Ref. [33] (again to be translated to dimensional regularization, also in the next two cases) and corresponds to (4.9) for small $m_{1}^{2}$. Integral (4.48) results from case (i) of Ref. [33] for $m_{2}^{2}=0, m_{3}^{2}=m_{4}^{2}$ and small $m_{1}=m_{0}$, or analogously from (4.4) with $p_{21}^{2}=0, p_{32}^{2}=m_{3}^{2}$ and $m_{1}^{2}=m_{2}^{2}$ small. Integral (4.49) results from case (i) of Ref. [33] for $m_{0}^{2}=m_{3}^{2}=m_{2}^{2}$ and both $m_{1}$ and $m_{4}$ small and corresponds to (4.4) with $p_{21}^{2}=p_{32}^{2}=m_{2}^{2}$ and both $m_{1}^{2}$ and $m_{3}^{2}$ small.

(iv) Cases with two collinear singularities on opposite legs $\left(n_{\text {coll }}=2\right)$

We distinguish seven different cases which do not contain a soft singularity and have the following common finite part

$$
D_{0}^{(\mathrm{fin})}=D_{0}-\frac{\left(p_{31}^{2}-p_{21}^{2}\right) C_{0}(0)+\left(p_{20}^{2}-p_{30}^{2}\right) C_{0}(1)+\left(p_{31}^{2}-p_{30}^{2}\right) C_{0}(2)+\left(p_{20}^{2}-p_{21}^{2}\right) C_{0}(3)}{p_{20}^{2} p_{31}^{2}-p_{21}^{2} p_{30}^{2}}
$$


The explicit results read

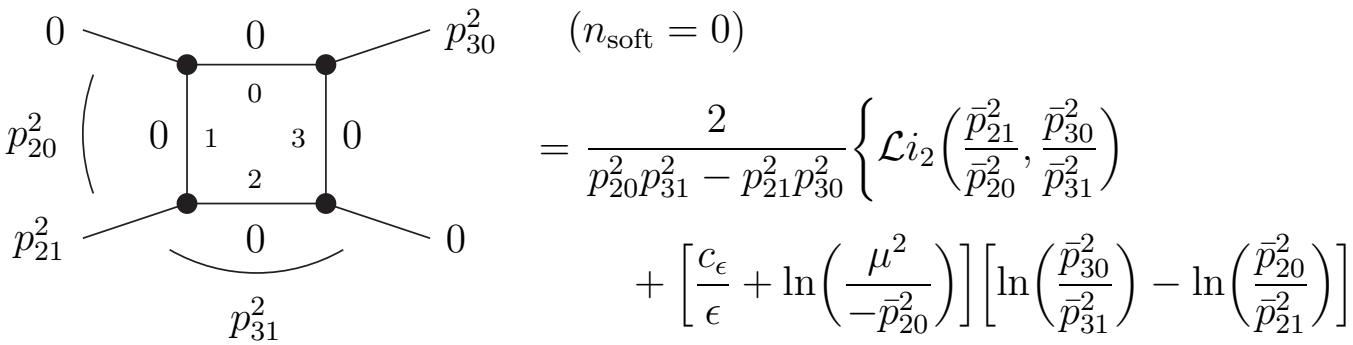

$$
\begin{aligned}
& \left.+\mathrm{Li}_{2}\left(1-\frac{\bar{p}_{20}^{2}}{\bar{p}_{21}^{2}}\right)+\mathrm{Li}_{2}\left(1-\frac{\bar{p}_{20}^{2}}{\bar{p}_{30}^{2}}\right)-\mathrm{Li}_{2}\left(1-\frac{\bar{p}_{21}^{2}}{\bar{p}_{31}^{2}}\right)-\mathrm{Li}_{2}\left(1-\frac{\bar{p}_{30}^{2}}{\bar{p}_{31}^{2}}\right)\right\},
\end{aligned}
$$

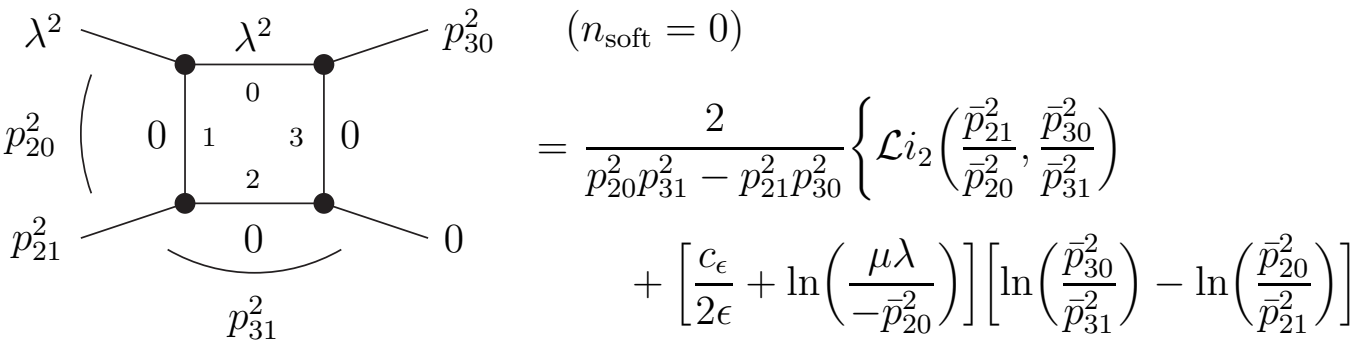

$$
\begin{aligned}
& \left.+\operatorname{Li}_{2}\left(1-\frac{\bar{p}_{20}^{2}}{\bar{p}_{30}^{2}}\right)-\operatorname{Li}_{2}\left(1-\frac{\bar{p}_{21}^{2}}{\bar{p}_{31}^{2}}\right)\right\}
\end{aligned}
$$

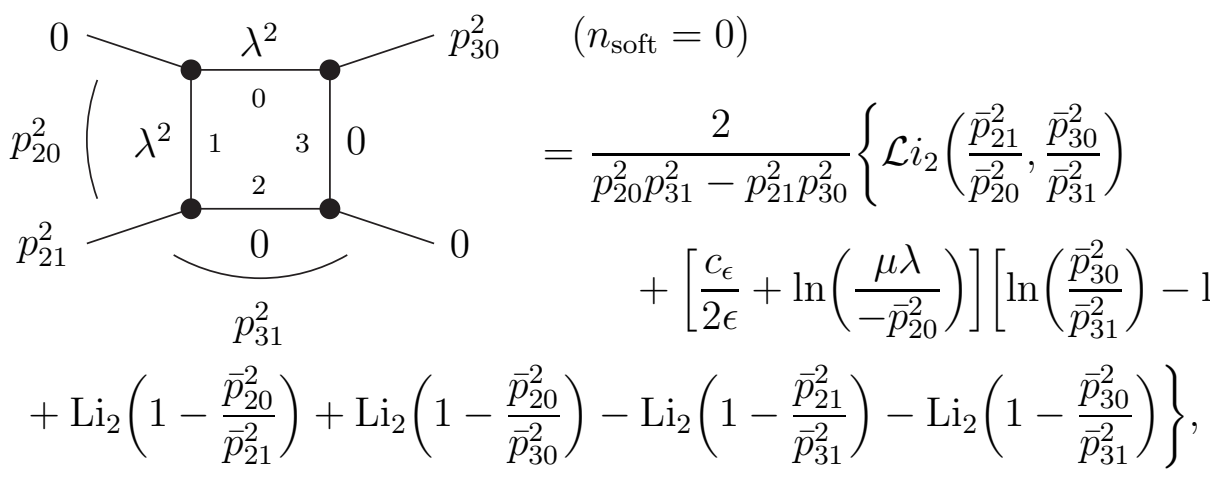

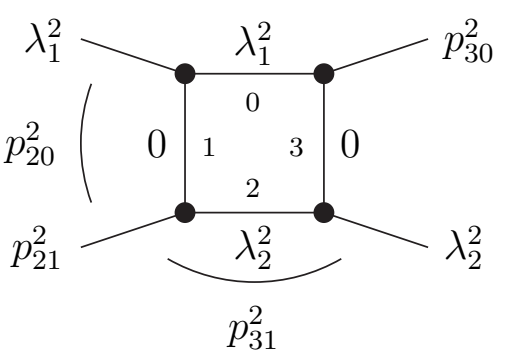

$$
\begin{aligned}
& \left(n_{\text {soft }}=0\right) \\
& =\frac{2}{p_{20}^{2} p_{31}^{2}-p_{21}^{2} p_{30}^{2}}\left\{\mathcal{L} i_{2}\left(\frac{\bar{p}_{21}^{2}}{\bar{p}_{20}^{2}}, \frac{\bar{p}_{30}^{2}}{\bar{p}_{31}^{2}}\right)\right. \\
& \left.\quad+\ln \left(\frac{\lambda_{1} \lambda_{2}}{-\bar{p}_{20}^{2}}\right)\left[\ln \left(\frac{\bar{p}_{30}^{2}}{\bar{p}_{31}^{2}}\right)-\ln \left(\frac{\bar{p}_{20}^{2}}{\bar{p}_{21}^{2}}\right)\right]\right\},
\end{aligned}
$$



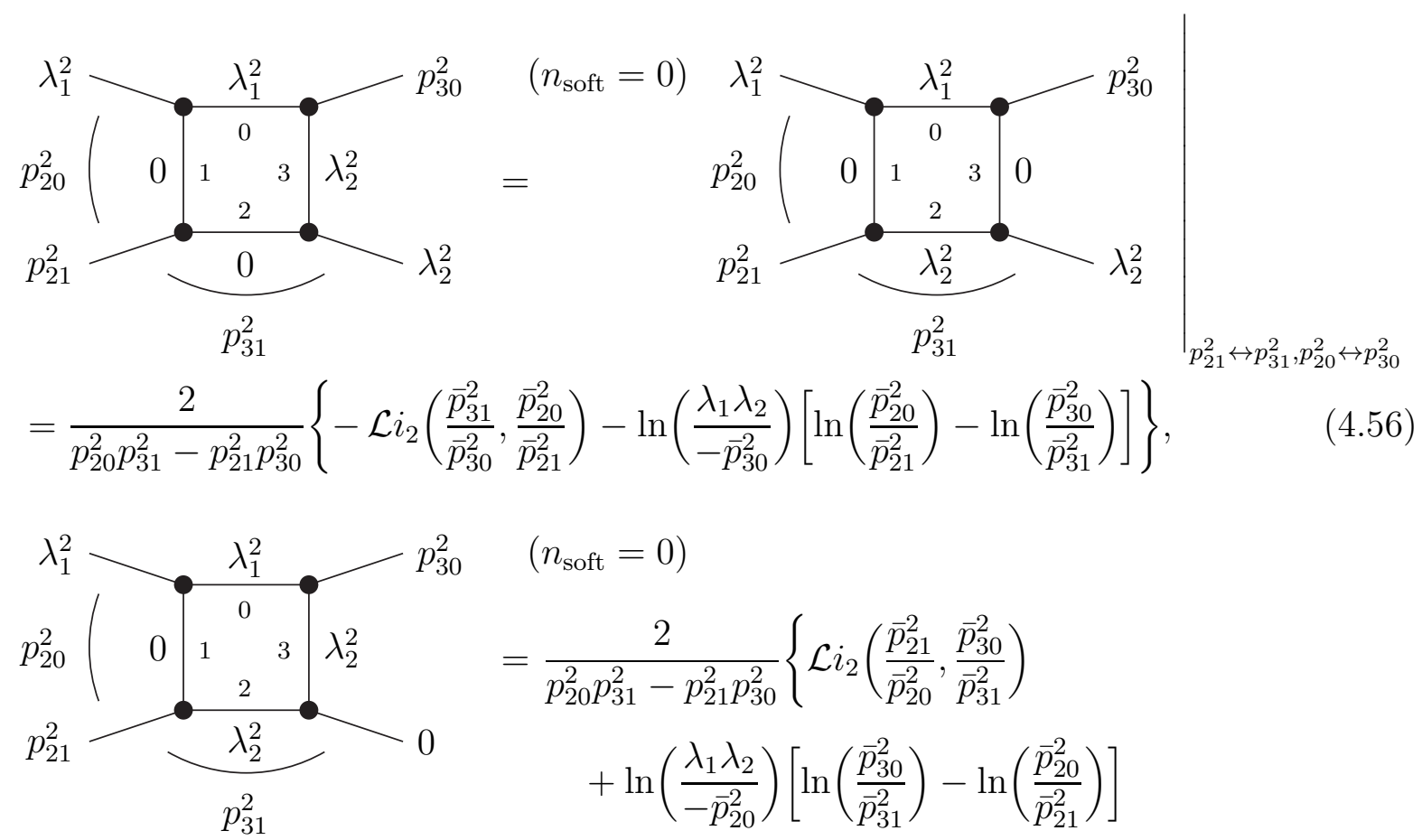

$\left.+\mathrm{Li}_{2}\left(1-\frac{\bar{p}_{20}^{2}}{\bar{p}_{30}^{2}}\right)-\mathrm{Li}_{2}\left(1-\frac{\bar{p}_{21}^{2}}{\bar{p}_{31}^{2}}\right)\right\}$

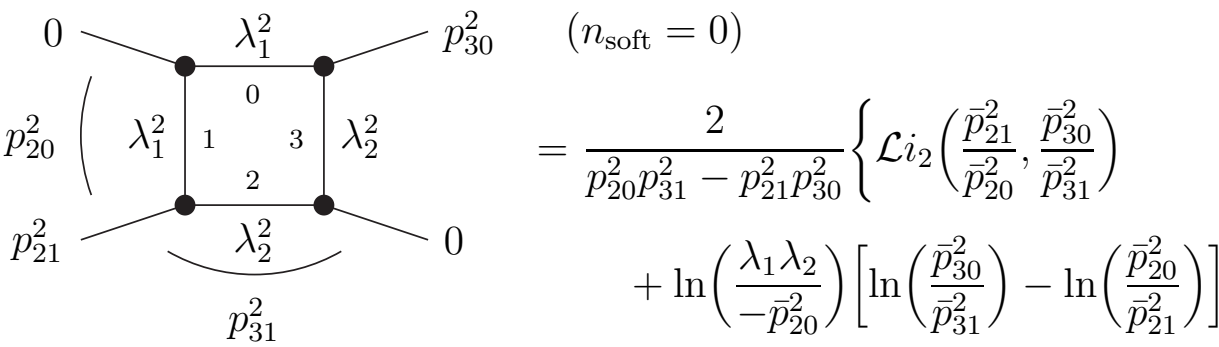

$$
\begin{aligned}
& \left.+\operatorname{Li}_{2}\left(1-\frac{\bar{p}_{20}^{2}}{\bar{p}_{21}^{2}}\right)+\operatorname{Li}_{2}\left(1-\frac{\bar{p}_{20}^{2}}{\bar{p}_{30}^{2}}\right)-\mathrm{Li}_{2}\left(1-\frac{\bar{p}_{21}^{2}}{\bar{p}_{31}^{2}}\right)-\mathrm{Li}_{2}\left(1-\frac{\bar{p}_{30}^{2}}{\bar{p}_{31}^{2}}\right)\right\} \text {. }
\end{aligned}
$$

The integral (4.52) can be found in Refs. [19, 26, 39] and corresponds to "Box 3" of Ref. [28]. Integrals (4.53)-(4.58) can be obtained from (4.13)-(4.15) with appropriate substitutions.

(v) Cases with three collinear singularities $\left(n_{\text {coll }}=3\right)$

There are ten different cases with up to two soft singularities and the following common finite part

$$
D_{0}^{(\mathrm{fin})}=D_{0}-\frac{C_{0}(0)}{p_{20}^{2}}-\frac{\left(p_{20}^{2}-p_{30}^{2}\right) C_{0}(1)+\left(p_{31}^{2}-p_{30}^{2}\right) C_{0}(2)}{p_{20}^{2} p_{31}^{2}}-\frac{C_{0}(3)}{p_{31}^{2}} .
$$




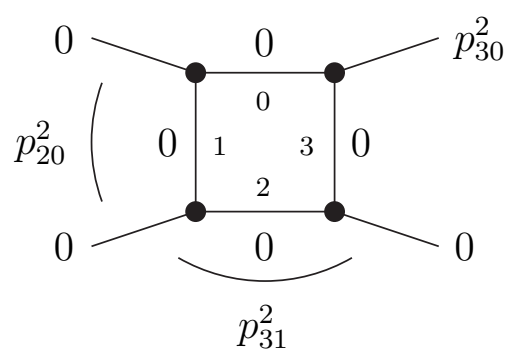

$$
\begin{aligned}
& \left(n_{\mathrm{soft}}=2\right) \\
& =\frac{1}{p_{20}^{2} p_{31}^{2}}\left\{\frac{2 c_{\epsilon}}{\epsilon^{2}}+\frac{2 c_{\epsilon}}{\epsilon}\left[\ln \left(\frac{\mu^{2}}{-\bar{p}_{20}^{2}}\right)+\ln \left(\frac{\bar{p}_{30}^{2}}{\bar{p}_{31}^{2}}\right)\right]\right. \\
& \quad+2 \ln \left(\frac{\mu^{2}}{-\bar{p}_{31}^{2}}\right) \ln \left(\frac{\mu^{2}}{-\bar{p}_{20}^{2}}\right)-\ln ^{2}\left(\frac{\mu^{2}}{-\bar{p}_{30}^{2}}\right) \\
& \left.\quad-2 \operatorname{Li}_{2}\left(1-\frac{\bar{p}_{30}^{2}}{\bar{p}_{20}^{2}}\right)-2 \operatorname{Li}_{2}\left(1-\frac{\bar{p}_{30}^{2}}{\bar{p}_{31}^{2}}\right)-\frac{2 \pi^{2}}{3}\right\}
\end{aligned}
$$

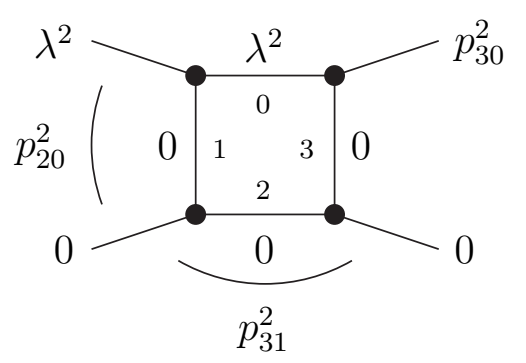

$$
\begin{aligned}
& \left(n_{\mathrm{soft}}=2\right) \\
& =\frac{1}{p_{20}^{2} p_{31}^{2}}\left\{\frac{3 c_{\epsilon}}{2 \epsilon^{2}}+\frac{c_{\epsilon}}{\epsilon}\left[2 \ln \left(\frac{\mu \lambda}{-\bar{p}_{20}^{2}}\right)-\ln \left(\frac{\mu \lambda}{-\bar{p}_{30}^{2}}\right)+\ln \left(\frac{\mu^{2}}{-\bar{p}_{31}^{2}}\right)\right]\right. \\
& \quad+2 \ln \left(\frac{\mu^{2}}{-\bar{p}_{31}^{2}}\right) \ln \left(\frac{\mu \lambda}{-\bar{p}_{20}^{2}}\right)-\ln ^{2}\left(\frac{\mu \lambda}{-\bar{p}_{30}^{2}}\right) \\
& \left.\quad-2 \operatorname{Li}_{2}\left(1-\frac{\bar{p}_{30}^{2}}{\bar{p}_{20}^{2}}\right)-\frac{2 \pi^{2}}{3}\right\},
\end{aligned}
$$

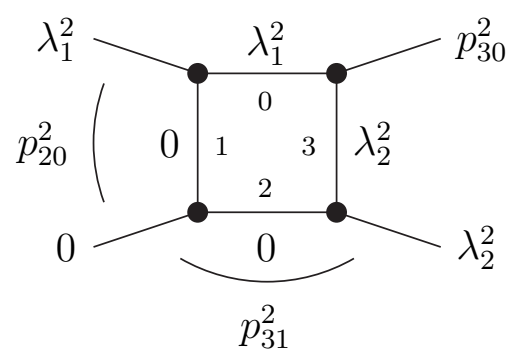

$$
\begin{aligned}
& \left(n_{\mathrm{soft}}=2\right) \\
& =\frac{1}{p_{20}^{2} p_{31}^{2}}\left\{\frac{c_{\epsilon}}{\epsilon^{2}}+\frac{c_{\epsilon}}{\epsilon}\left[\ln \left(\frac{\mu \lambda_{1}}{-\bar{p}_{20}^{2}}\right)+\ln \left(\frac{\mu \lambda_{2}}{-\bar{p}_{31}^{2}}\right)\right]\right. \\
& \left.\quad+2 \ln \left(\frac{\mu \lambda_{1}}{-\bar{p}_{20}^{2}}\right) \ln \left(\frac{\mu \lambda_{2}}{-\bar{p}_{31}^{2}}\right)-\ln ^{2}\left(\frac{\lambda_{1} \lambda_{2}}{-\bar{p}_{30}^{2}}\right)-\frac{2 \pi^{2}}{3}\right\},
\end{aligned}
$$

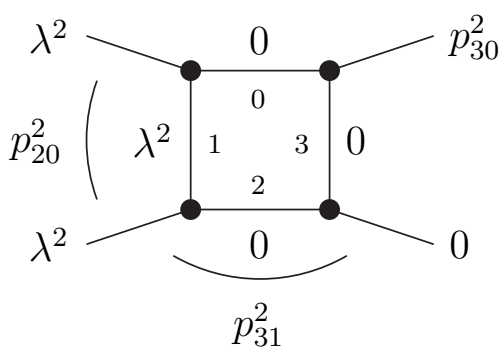

$$
\begin{aligned}
& \left(n_{\mathrm{soft}}=1\right) \\
& =\frac{1}{p_{20}^{2} p_{31}^{2}}\left\{\frac{c_{\epsilon}}{2 \epsilon^{2}}+\frac{c_{\epsilon}}{\epsilon}\left[\ln \left(\frac{\mu \lambda}{-\bar{p}_{31}^{2}}\right)+\ln \left(\frac{\bar{p}_{30}^{2}}{\bar{p}_{20}^{2}}\right)\right]\right. \\
& \left.\quad+\left[\ln \left(\frac{\mu \lambda}{-\bar{p}_{31}^{2}}\right)+\ln \left(\frac{\bar{p}_{30}^{2}}{\bar{p}_{20}^{2}}\right)\right]^{2}+2 \operatorname{Li}_{2}\left(1-\frac{\bar{p}_{20}^{2}}{\bar{p}_{30}^{2}}\right)+\frac{\pi^{2}}{6}\right\},
\end{aligned}
$$




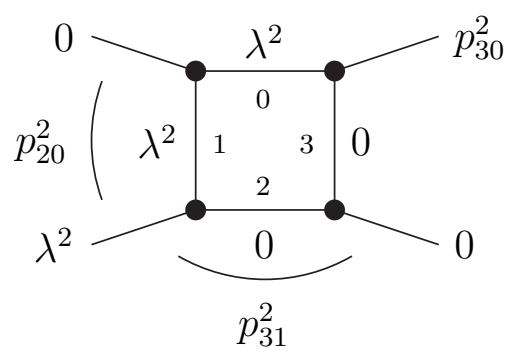

$$
\begin{aligned}
& \left(n_{\text {soft }}=1\right) \\
& =\frac{1}{p_{20}^{2} p_{31}^{2}}\left\{\frac{c_{\epsilon}}{2 \epsilon^{2}}+\frac{c_{\epsilon}}{\epsilon}\left[\ln \left(\frac{\mu \lambda}{-\bar{p}_{20}^{2}}\right)+\ln \left(\frac{\bar{p}_{30}^{2}}{\bar{p}_{31}^{2}}\right)\right]\right. \\
& \quad+2 \ln \left(\frac{\mu \lambda}{-\bar{p}_{20}^{2}}\right) \ln \left(\frac{\mu \lambda}{-\bar{p}_{31}^{2}}\right)-\ln ^{2}\left(\frac{\mu \lambda}{\bar{p}_{30}^{2}}\right) \\
& \left.\quad-2 \operatorname{Li}_{2}\left(1-\frac{\bar{p}_{30}^{2}}{\bar{p}_{20}^{2}}\right)-2 \operatorname{Li}_{2}\left(1-\frac{\bar{p}_{30}^{2}}{\bar{p}_{31}^{2}}\right)-\frac{\pi^{2}}{6}\right\}
\end{aligned}
$$

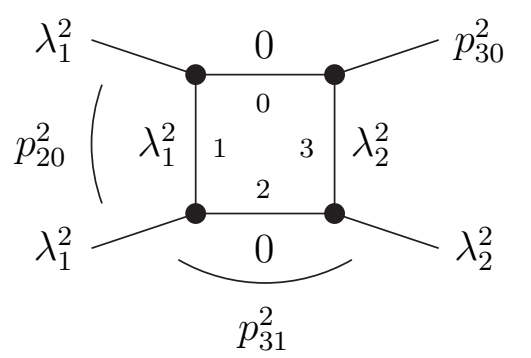

$$
\begin{aligned}
& \left(n_{\mathrm{soft}}=1\right) \\
& =\frac{1}{p_{20}^{2} p_{31}^{2}}\left\{\operatorname { l n } ( \frac { \lambda _ { 1 } \lambda _ { 2 } } { - \overline { p } _ { 3 1 } ^ { 2 } } ) \left[\frac{c_{\epsilon}}{\epsilon}+\ln \left(\frac{\lambda_{1} \lambda_{2}}{-\bar{p}_{31}^{2}}\right)-2 \ln \left(\frac{\lambda_{2} \mu}{-\bar{p}_{30}^{2}}\right)\right.\right. \\
& \left.\left.\quad+2 \ln \left(\frac{\mu^{2}}{-\bar{p}_{20}^{2}}\right)\right]+\frac{\pi^{2}}{6}\right\}
\end{aligned}
$$

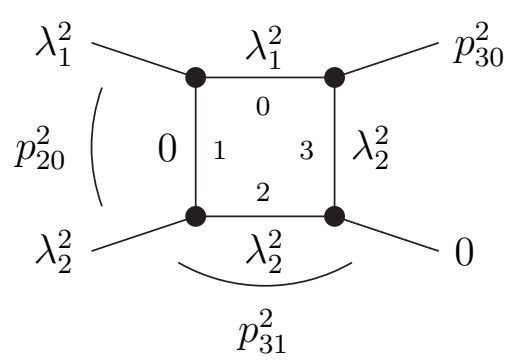

$$
\begin{aligned}
& \left(n_{\mathrm{soft}}=1\right) \\
& =\frac{1}{p_{20}^{2} p_{31}^{2}}\left\{\operatorname { l n } ( \frac { \lambda _ { 1 } \lambda _ { 2 } } { - \overline { p } _ { 2 0 } ^ { 2 } } ) \left[\frac{c_{\epsilon}}{\epsilon}+\ln \left(\frac{\lambda_{1} \lambda_{2}}{-\bar{p}_{20}^{2}}\right)-2 \ln \left(\frac{\lambda_{1} \lambda_{2}}{-\bar{p}_{30}^{2}}\right)\right.\right. \\
& \left.\left.\quad+2 \ln \left(\frac{\lambda_{2} \mu}{-\bar{p}_{31}^{2}}\right)\right]+2 \operatorname{Li}_{2}\left(1-\frac{\bar{p}_{20}^{2}}{\bar{p}_{30}^{2}}\right)-\frac{\pi^{2}}{6}\right\}
\end{aligned}
$$

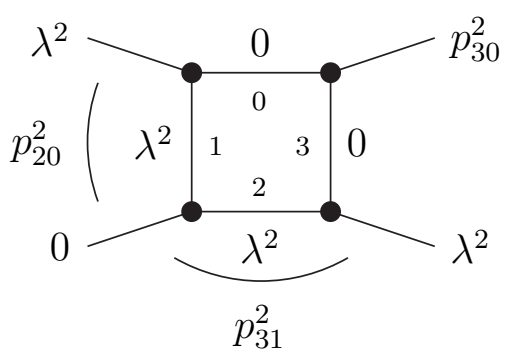

$$
\begin{aligned}
& \left(n_{\text {soft }}=0\right) \\
= & \frac{1}{p_{20}^{2} p_{31}^{2}}\left\{\left[\ln \left(\frac{\lambda^{2}}{-\bar{p}_{20}^{2}}\right)+\ln \left(\frac{\bar{p}_{30}^{2}}{\bar{p}_{31}^{2}}\right)\right]^{2}+\frac{\pi^{2}}{3}\right\},
\end{aligned}
$$

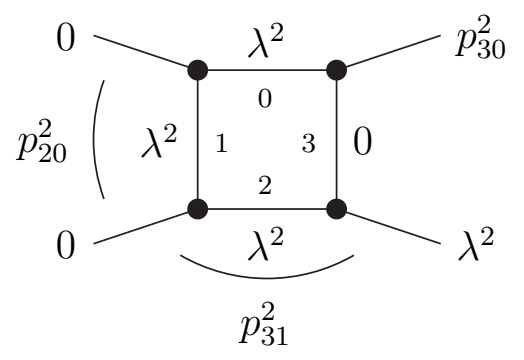

$$
\begin{aligned}
& \left(n_{\mathrm{soft}}=0\right) \\
= & \frac{1}{p_{20}^{2} p_{31}^{2}}\left\{\left[\ln \left(\frac{\lambda^{2}}{-\bar{p}_{20}^{2}}\right)+\ln \left(\frac{\bar{p}_{30}^{2}}{\bar{p}_{31}^{2}}\right)\right]^{2}+2 \operatorname{Li}_{2}\left(1-\frac{\bar{p}_{31}^{2}}{\bar{p}_{30}^{2}}\right)\right\}
\end{aligned}
$$




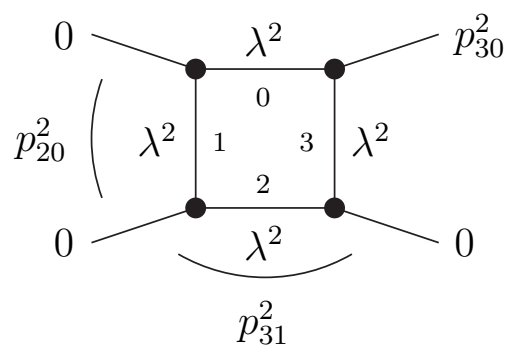

$$
\begin{aligned}
& \left(n_{\mathrm{soft}}=0\right) \\
& =\frac{1}{p_{20}^{2} p_{31}^{2}}\left\{\left[\ln \left(\frac{\lambda^{2}}{-\bar{p}_{20}^{2}}\right)+\ln \left(\frac{\bar{p}_{30}^{2}}{\bar{p}_{31}^{2}}\right)\right]^{2}\right. \\
& \left.\quad+2 \operatorname{Li}_{2}\left(1-\frac{\bar{p}_{20}^{2}}{\bar{p}_{30}^{2}}\right)+2 \operatorname{Li}_{2}\left(1-\frac{\bar{p}_{31}^{2}}{\bar{p}_{30}^{2}}\right)-\frac{\pi^{2}}{3}\right\} .
\end{aligned}
$$

The integral (4.60) can be found in Refs. [ 19, 26, 39], corresponds to "Box 2" of Ref. [ 28], and had already been calculated in Refs. [ 24, 25]. Integral (4.61) can be obtained from (A.4) of Ref. [35] and corresponds to (4.36) with small $m_{3}$. Integral (4.62) corresponds to (4.25) with both $m_{2}$ and $m_{3}$ small. Integral (4.63) can be obtained from (4.19) for $m_{2}^{2}=0$, $p_{32}^{2}=m_{3}^{2}$, and $m_{3}^{2}$ small. Integral (4.64) is a particular case of (4.19). Integrals (4.65) and (4.66) are cases (iid) and (id) of Ref. [33] (translated to dimensional regularization), respectively, and can also be derived from (4.39) and (4.40) for small $m_{3}$, respectively.

(vi) Cases with four collinear singularities $\left(n_{\text {coll }}=4\right)$

There are six different cases with up to four soft singularities and the following common finite part

$$
D_{0}^{(\mathrm{fin})}=D_{0}-\frac{C_{0}(0)+C_{0}(2)}{p_{20}^{2}}-\frac{C_{0}(1)+C_{0}(3)}{p_{31}^{2}}
$$

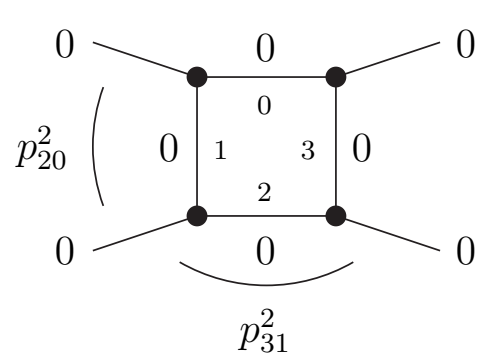

$$
\begin{aligned}
& \left(n_{\text {soft }}=4\right) \\
& =\frac{1}{p_{20}^{2} p_{31}^{2}}\left\{\frac{4 c_{\epsilon}}{\epsilon^{2}}+\frac{2 c_{\epsilon}}{\epsilon}\left[\ln \left(\frac{\mu^{2}}{-\bar{p}_{20}^{2}}\right)+\ln \left(\frac{\mu^{2}}{-\bar{p}_{31}^{2}}\right)\right]\right. \\
& \left.\quad+2 \ln \left(\frac{\mu^{2}}{-\bar{p}_{31}^{2}}\right) \ln \left(\frac{\mu^{2}}{-\bar{p}_{20}^{2}}\right)-\frac{5 \pi^{2}}{3}\right\},
\end{aligned}
$$

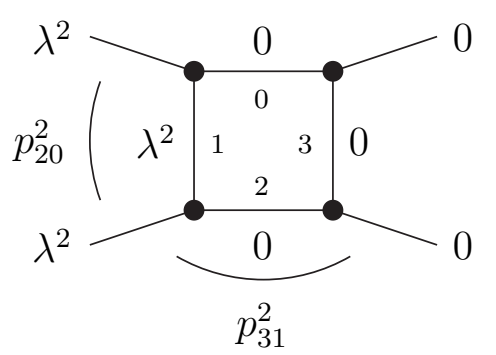

$$
\begin{aligned}
& \left(n_{\text {soft }}=3\right) \\
& =\frac{1}{p_{20}^{2} p_{31}^{2}}\left\{\frac{2 c_{\epsilon}}{\epsilon^{2}}+\frac{c_{\epsilon}}{\epsilon}\left[\ln \left(\frac{\mu^{2}}{-\bar{p}_{20}^{2}}\right)+2 \ln \left(\frac{\mu \lambda}{-\bar{p}_{31}^{2}}\right)\right]\right. \\
& \left.\quad+2 \ln \left(\frac{\mu \lambda}{-\bar{p}_{31}^{2}}\right) \ln \left(\frac{\mu^{2}}{-\bar{p}_{20}^{2}}\right)-\frac{5 \pi^{2}}{6}\right\},
\end{aligned}
$$

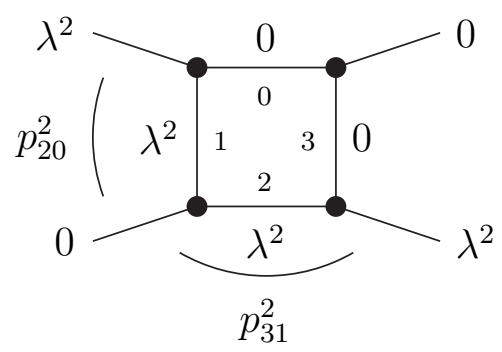

$$
\begin{aligned}
& \left(n_{\mathrm{soft}}=2\right) \\
& =\frac{1}{p_{20}^{2} p_{31}^{2}}\left\{\frac{c_{\epsilon}}{\epsilon^{2}}+\frac{c_{\epsilon}}{\epsilon}\left[\ln \left(\frac{\mu \lambda}{-\bar{p}_{20}^{2}}\right)+\ln \left(\frac{\mu \lambda}{-\bar{p}_{31}^{2}}\right)\right]\right. \\
& \left.\quad+2 \ln \left(\frac{\mu \lambda}{-\bar{p}_{20}^{2}}\right) \ln \left(\frac{\mu \lambda}{-\bar{p}_{31}^{2}}\right)-\frac{2 \pi^{2}}{3}\right\},
\end{aligned}
$$



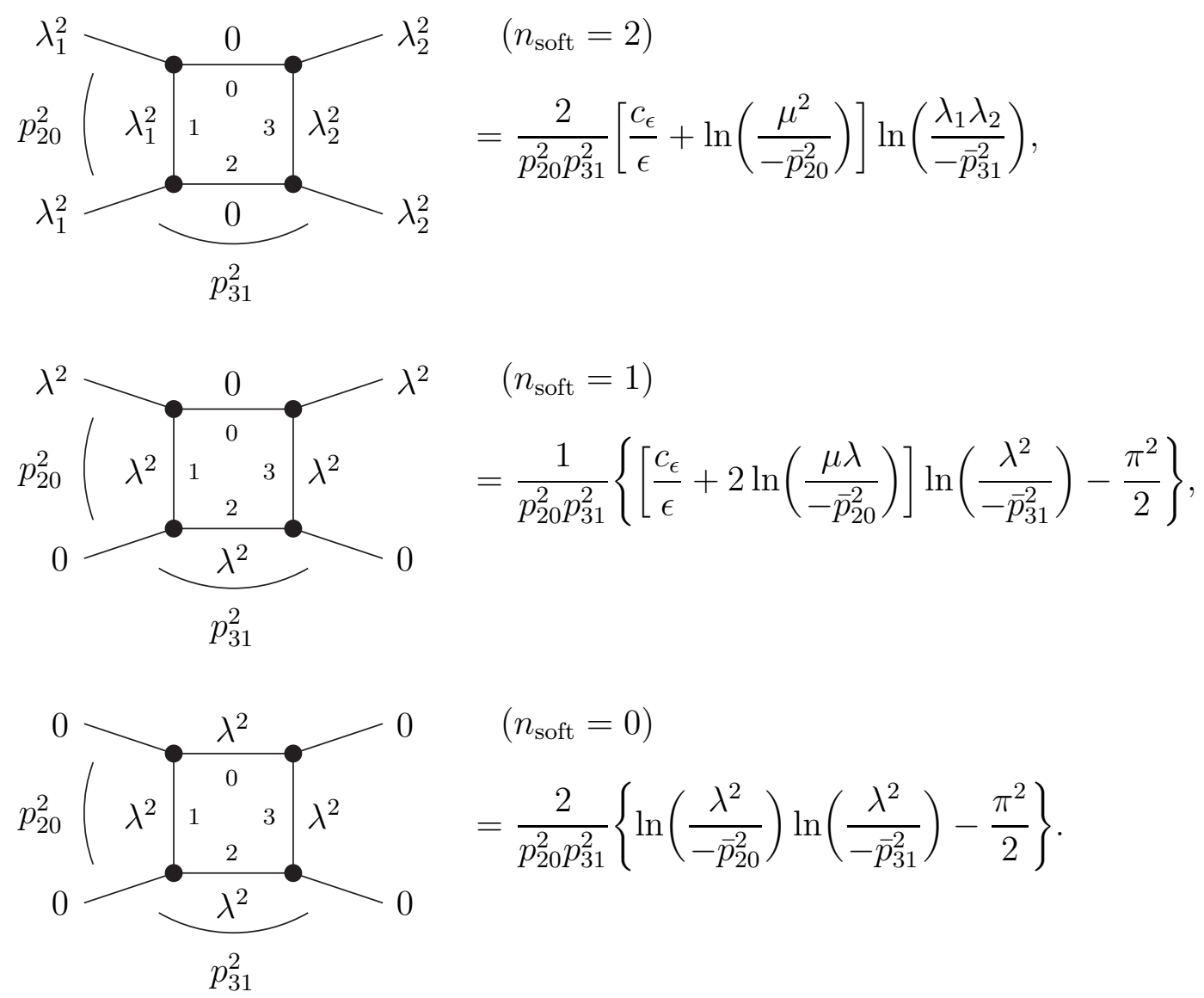

$$
\begin{aligned}
& \left(n_{\mathrm{soft}}=0\right) \\
= & \frac{2}{p_{20}^{2} p_{31}^{2}}\left\{\ln \left(\frac{\lambda^{2}}{-\bar{p}_{20}^{2}}\right) \ln \left(\frac{\lambda^{2}}{-\bar{p}_{31}^{2}}\right)-\frac{\pi^{2}}{2}\right\} .
\end{aligned}
$$

The integral (4.71) is given in (4.25) of Ref. [19], corresponds to "Box 1" of Ref. [28] and had already been calculated in Refs. [24, 25]. Integral (4.72) can be obtained from Ref. [38] and corresponds to (4.45) for small $m_{3}$. Integral (4.73) is a special case of (4.25) for $p_{32}^{2}=0$ and small masses. Integral (4.74) is case (iii b) of Ref. [33] (in dimensional regularization) and corresponds to (4.47) for small $m_{3}$. Integral (4.75) is case (ie) of Ref. [33] (in dimensional regularization) and corresponds to a special case of (4.4), for vanishing $p_{21}^{2}$ and $p_{32}^{2}$ and if again all masses are small.

\section{Summary}

The calculation of scalar one-loop integrals is at the heart of any perturbative calculation of radiative corrections at the one-loop level, independent of the method employed. In diagrammatic calculations, which do not rely on numerical integration of loop integrals, tensor integrals as well as any one-loop integral with more than four external particles (legs) are algebraically reduced to standard scalar integrals with at most four propagators. Recently so-called unitarity-based methods became more and more popular at the one-loop level, i.e. methods that abandon the direct use of Feynman diagrams, but also those methods need the standard scalar integrals with up to four propagators as input. The purpose of this paper is to close some gaps in the literature and to deliver a complete set of 4-point ("box") scalar integrals that appear in any kind of one-loop calculation. For the lower-point integrals exhaustive lists of results already exist. 
The considered scalar box integrals are naturally categorised into finite, regular cases and singular cases that involve soft and/or collinear divergences. For the most general regular case with complex internal masses, which appear in applications that involve unstable intermediate particles, we have presented two new analytical expressions that involve less (72 and 32) dilogarithmic functions than a recently published independent result of other authors. In addition we have listed a complete set of soft- and/or collinear-singular cases which appear in the calculation of strong or electroweak radiative corrections, i.e. we support both dimensional regularization and regularizations schemes that employ small mass parameters. Mixed regularizations of these types are supported as well, and all results are valid for complex masses of internal particles. Many of those results have not yet been published elsewhere.

In combination with available results on scalar 1-, 2-, and 3-point integrals now all relevant standard scalar integrals for one-loop calculations in QCD, QED, the electroweak Standard Model and typical extensions thereof are known for all not too exotic (mass or dimensional) regularization schemes, covering also internal unstable particles appearing as resonances.

\section{Acknowledgements}

This work is supported in part by the European Community's Marie-Curie Research Training Network under contract MRTN-CT-2006-035505 "Tools and Precision Calculations for Physics Discoveries at Colliders".

\section{References}

[1] F. del Aguila and R. Pittau, JHEP 0407 (2004) 017 hep-ph/0404120.

[2] T. Binoth, J. P. Guillet, G. Heinrich, E. Pilon and C. Schubert, JHEP 0510 (2005) 015 arXiv:hep-ph/0504267.

[3] R. K. Ellis, W. T. Giele and G. Zanderighi, Phys. Rev. D 73 (2006) 014027 arXiv:hep-ph/0508308.

[4] A. Denner and S. Dittmaier, Nucl. Phys. B 734 (2006) 62 arXiv:hep-ph/0509141.

[5] G. Ossola, C. G. Papadopoulos and R. Pittau, Nucl. Phys. B 763, 147 (2007) arXiv:hep-ph/0609007].

[6] Z. Bern, L. J. Dixon and D. A. Kosower, Annals Phys. 322, 1587 (2007) arXiv:0704.2798 [hep-ph]].

[7] R. K. Ellis, W. T. Giele and Z. Kunszt, JHEP 0803, 003 (2008) arXiv:0708.2398 [hep-ph]].

[8] C. F. Berger et al., Phys. Rev. D 78 (2008) 036003 arXiv:0803.4180 [hep-ph]].

[9] A. van Hameren, JHEP 0907 (2009) 088 [arXiv:0905.1005 [hep-ph]]. 
[10] C. Buttar et al. [QCD, EW, and Higgs Working Group], arXiv:hep-ph/0604120;

Z. Bern et al. [NLO Multileg Working Group], arXiv:0803.0494 [hep-ph];

T. Binoth et al. [SM and NLO Multileg Working Group], arXiv:1003.1241 [hep-ph].

[11] A. Ferroglia, M. Passera, G. Passarino and S. Uccirati, Nucl. Phys. B 650, 162 (2003) hep-ph/0209219.

[12] Z. Nagy and D. E. Soper, JHEP 0309 (2003) 055 [hep-ph/0308127].

[13] Y. Kurihara and T. Kaneko, Comput. Phys. Commun. 174 (2006) 530 arXiv:hep-ph/0503003].

[14] S. Catani, T. Gleisberg, F. Krauss, G. Rodrigo and J. C. Winter, JHEP 0809 (2008) 065 [arXiv:0804.3170 [hep-ph]].

[15] W. Kilian and T. Kleinschmidt, arXiv:0912.3495 [hep-ph].

[16] T. Binoth, G. Heinrich and N. Kauer, Nucl. Phys. B 654 (2003) 277 hep-ph/0210023.

[17] D. B. Melrose, Nuovo Cimento XL A (1965) 181.

[18] G. 't Hooft and M. J. Veltman, Nucl. Phys. B 153 (1979) 365.

[19] Z. Bern, L. J. Dixon and D. A. Kosower, Nucl. Phys. B 412 (1994) 751 hep-ph/9306240.

[20] A. Denner, S. Dittmaier, M. Roth and L. H. Wieders, Nucl. Phys. B 724 (2005) 247 hep-ph/0505042.

[21] T. N. Dao and D. N. Le, Comput. Phys. Commun. 180 (2009) 2258 arXiv:0902.0325 [hep-ph]].

[22] A. Denner, U. Nierste and R. Scharf, Nucl. Phys. B 367 (1991) 637.

[23] S. Dittmaier, Nucl. Phys. B 675 (2003) 447 hep-ph/0308246.

[24] K. Fabricius and I. Schmitt, Z. Phys. C 3 (1979) 51.

[25] S. Papadopoulos, A. P. Contogouris and J. Ralston, Phys. Rev. D 25 (1982) 2218.

[26] G. Duplancic and B. Nizic, Eur. Phys. J. C 20 (2001) 357 arXiv:hep-ph/0006249.

[27] G. Duplancic and B. Nizic, Eur. Phys. J. C 24 (2002) 385 arXiv:hep-ph/0201306.

[28] R. K. Ellis and G. Zanderighi, JHEP 0802 (2008) 002 [arXiv:0712.1851 [hep-ph]].

[29] A. Denner, S. Dittmaier and T. Hahn, Phys. Rev. D 56 (1997) 117 arXiv:hep-ph/9612390.

[30] F. Boudjema and L. D. Ninh, Phys. Rev. D 78 (2008) 093005 arXiv:0806.1498 [hep$\mathrm{ph}]]$. 
[31] G. Passarino, C. Sturm and S. Uccirati, Nucl. Phys. B 834 (2010) 77 arXiv:1001.3360 [hep-ph]].

[32] R. Lewin, Polylogarithms and associated functions (North-Holland, Amsterdam, 1981).

[33] W. Beenakker and A. Denner, Nucl. Phys. B 338 (1990) 349.

[34] T. Kinoshita, J. Math. Phys. 3 (1962) 650.

[35] W. Beenakker et al., Nucl. Phys. B 653 (2003) 151 hep-ph/0211352.

[36] P. Van Nieuwenhuizen, Nucl. Phys. B 28 (1971) 429;

F. A. Berends, K. J. F. Gaemers and R. Gastmans, Nucl. Phys. B 63 (1973) 381.

[37] E. L. Berger, M. Klasen and T. M. P. Tait, Phys. Rev. D 62, 095014 (2000) arXiv:hep-ph/0005196].

[38] W. Beenakker, H. Kuijf, W. L. van Neerven and J. Smith, Phys. Rev. D 40 (1989) 54 .

[39] R. K. Ellis, D. A. Ross and A. E. Terrano, Nucl. Phys. B 178, 421 (1981). 